\title{
Synthesis of Tumor-Associated $\mathrm{Le}^{\mathrm{a}} \mathrm{Le}^{\mathrm{x}}$ hexasaccharides: Instability of a Thiol-containing Oligosaccharide in Mass Spectrometry and Hyper-metalation detected by ESI FAIMS.
}

\author{
Mickaël Guillemineau, Jadwiga Lyczko, Wojciech Gabryelski, France-Isabelle Auzanneau* \\ Department of Chemistry, University of Guelph, Guelph, Ontario, N1G 2WI, Canada \\ Corresponding author. Tel: 01-519-824-4120, ext. 53809; fax: 01-519-766-1499; \\ e-mail: fauzanne@uoguelph.ca
}

Supporting Information

page

Tables 1-4: listing of ions detected by ESI FAIMS HRMS for compounds 2-4, 23

$2-5$

ESI FAIMS HRMS spectra for compounds 2-4, 23

6-9

ESI HRMS for compounds 2-4

10-12

General Experimental Procedures

${ }^{1} \mathrm{H}-$ and ${ }^{13} \mathrm{C}$ - NMR spectra for new compounds

14-73 
Table 1: listing of ions detected by ESI FAIMS HRMS for hexasaccharide 2

\begin{tabular}{ccccc}
\hline Ion & $\begin{array}{c}\text { Molecular } \\
\text { Formula }\end{array}$ & $\begin{array}{c}\text { Calculated } \\
\mathrm{m} / z\end{array}$ & $\begin{array}{c}\text { Measured } \\
\text { Accurate } \\
m / z\end{array}$ & $\begin{array}{c}\text { mass error } \\
(\mathrm{ppm})\end{array}$ \\
\hline$[\mathrm{M}+2 \mathrm{H}]^{2+}$ & $\mathrm{C}_{46} \mathrm{H}_{82} \mathrm{~N}_{2} \mathrm{O}_{29}$ & 563.2496 & 563.2512 & 3 \\
{$\left[\mathrm{M}+\mathrm{H}+\mathrm{NH}_{4}\right]^{2+}$} & $\mathrm{C}_{46} \mathrm{H}_{85} \mathrm{~N}_{3} \mathrm{O}_{29}$ & 571.7629 & 571.7635 & 1 \\
{$\left[\mathrm{M}+2 \mathrm{NH}_{4}\right]^{2+}$} & $\mathrm{C}_{46} \mathrm{H}_{89} \mathrm{~N}_{4} \mathrm{O}_{29}$ & 580.2762 & 580.2776 & 2 \\
{$[\mathrm{M}+\mathrm{H}+\mathrm{Na}]^{2+}$} & $\mathrm{C}_{46} \mathrm{H}_{81} \mathrm{~N}_{2} \mathrm{O}_{29} \mathrm{Na}$ & 574.2406 & 574.2431 & 4 \\
{$[\mathrm{M}+2 \mathrm{Na}]^{2+}$} & $\mathrm{C}_{46} \mathrm{H}_{80} \mathrm{~N}_{2} \mathrm{O}_{29} \mathrm{Na}_{2}$ & 585.2316 & 585.2345 & 5 \\
\hline
\end{tabular}


Table 2: listing of ions detected by ESI FAIMS HRMS for hexasaccharide 3

\begin{tabular}{ccccc}
\hline Ion & $\begin{array}{c}\text { Molecular } \\
\text { Formula }\end{array}$ & $\begin{array}{c}\text { Calculated } \\
m / z\end{array}$ & $\begin{array}{c}\text { Measured } \\
\text { Accurate }^{a} \\
m / z\end{array}$ & $\begin{array}{c}\text { mass error } \\
(\mathrm{ppm})\end{array}$ \\
\hline$[\mathrm{M}+2 \mathrm{H}]^{2+}$ & $\mathrm{C}_{46} \mathrm{H}_{83} \mathrm{~N}_{3} \mathrm{O}_{29}$ & 570.7551 & 570.7554 & 1 \\
{$\left[\mathrm{M}+\mathrm{H}+\mathrm{NH}_{4}\right]^{2+}$} & $\mathrm{C}_{46} \mathrm{H}_{86} \mathrm{~N}_{4} \mathrm{O}_{29}$ & 579.2683 & 579.2693 & 2 \\
{$[\mathrm{M}+\mathrm{H}+\mathrm{Na}]^{2+}$} & $\mathrm{C}_{46} \mathrm{H}_{82} \mathrm{~N}_{3} \mathrm{O}_{29} \mathrm{Na}$ & 581.7460 & 581.7467 & 1 \\
\hline
\end{tabular}


Table 3: listing of ions detected by ESI FAIMS HRMS for hexasaccharide 4

\begin{tabular}{ccccc}
\hline Ion & $\begin{array}{c}\text { Molecular } \\
\text { Formula }\end{array}$ & $\begin{array}{c}\text { Calculated } \\
m / z\end{array}$ & $\begin{array}{c}\text { Measured } \\
\text { Accurate } \\
m / z\end{array}$ & $\begin{array}{c}\text { mass error } \\
\text { (ppm) }\end{array}$ \\
\hline$\left[\mathrm{M}-\mathrm{H}_{2} \mathrm{O}-\mathrm{H}+3 \mathrm{Na}\right]^{2+}$ & $\mathrm{C}_{46} \mathrm{H}_{77} \mathrm{~N}_{2} \mathrm{O}_{28} \mathrm{SNa}_{3}$ & 603.2033 & 603.2016 & -3 \\
{$\left[\mathrm{M}-\mathrm{H}_{2} \mathrm{O}-2 \mathrm{H}+4 \mathrm{Na}\right]^{2+}$} & $\mathrm{C}_{46} \mathrm{H}_{76} \mathrm{~N}_{2} \mathrm{O}_{28} \mathrm{SNa}_{4}$ & 614.1943 & 614.1937 & -1 \\
{$\left[\mathrm{M}-\mathrm{H}_{2} \mathrm{O}-3 \mathrm{H}+5 \mathrm{Na}\right]^{2+}$} & $\mathrm{C}_{46} \mathrm{H}_{75} \mathrm{~N}_{2} \mathrm{O}_{28} \mathrm{SNa}_{5}$ & 625.1852 & 625.1863 & \\
{$\left[\mathrm{M}-\mathrm{H}_{2} \mathrm{O}-4 \mathrm{H}+6 \mathrm{Na}\right]^{2+}$} & $\mathrm{C}_{46} \mathrm{H}_{74} \mathrm{~N}_{2} \mathrm{O}_{28} \mathrm{SNa}_{6}$ & 636.1762 & 636.1795 & 5 \\
{$\left[\mathrm{M}-\mathrm{H}_{2} \mathrm{O}-2 \mathrm{H}+3 \mathrm{Na}+\mathrm{NH}_{4}\right]^{2+}$} & $\mathrm{C}_{46} \mathrm{H}_{80} \mathrm{~N}_{3} \mathrm{O}_{28} \mathrm{SNa}_{3}$ & 611.7171 & 611.7159 & -2 \\
{$\left[\mathrm{M}-\mathrm{H}_{2} \mathrm{O}-3 \mathrm{H}+4 \mathrm{Na}+\mathrm{NH}_{4}\right]^{2+}$} & $\mathrm{C}_{46} \mathrm{H}_{79} \mathrm{~N}_{3} \mathrm{O}_{28} \mathrm{SNa}_{4}$ & 622.7075 & 622.7076 & 0 \\
{$\left[\mathrm{M}-\mathrm{H}_{2} \mathrm{O}-3 \mathrm{H}+3 \mathrm{Na}+2 \mathrm{NH}_{4}\right]^{2+}$} & $\mathrm{C}_{46} \mathrm{H}_{83} \mathrm{~N}_{4} \mathrm{O}_{28} \mathrm{SNa}_{3}$ & 620.2298 & 620.2292 & -1 \\
\hline
\end{tabular}


Table 4: listing of ions detected by ESI FAIMS HRMS for LeX disulfide 23

\begin{tabular}{ccccc}
\hline Ion & $\begin{array}{c}\text { Molecular } \\
\text { Formula }\end{array}$ & $\begin{array}{c}\text { Calculated } \\
m / z\end{array}$ & $\begin{array}{c}\text { Measured } \\
\text { Accurate } \\
m / z\end{array}$ & $\begin{array}{c}\text { mass error } \\
(\mathrm{ppm})\end{array}$ \\
\hline$[\mathrm{M}+2 \mathrm{H}]^{2+}$ & $\mathrm{C}_{52} \mathrm{H}_{94} \mathrm{~N}_{2} \mathrm{O}_{30} \mathrm{~S}_{2}$ & 645.2661 & 645.2682 & 3 \\
{$\left[\mathrm{M}+\mathrm{H}+\mathrm{NH}_{4}\right]^{2+}$} & $\mathrm{C}_{52} \mathrm{H}_{97} \mathrm{~N}_{3} \mathrm{O}_{30} \mathrm{~S}_{2}$ & 653.7794 & 653.7820 & 4 \\
{$\left[\mathrm{M}+2 \mathrm{NH}_{4}\right]^{2+}$} & $\mathrm{C}_{52} \mathrm{H}_{100} \mathrm{~N}_{4} \mathrm{O}_{30} \mathrm{~S}_{2}$ & 662.2927 & 662.2942 & 2 \\
{$[\mathrm{M}+\mathrm{H}+\mathrm{Na}]^{2+}$} & $\mathrm{C}_{52} \mathrm{H}_{93} \mathrm{~N}_{2} \mathrm{O}_{30} \mathrm{~S}_{2} \mathrm{Na}$ & 656.2571 & 656.2581 & 2 \\
{$[\mathrm{M}+2 \mathrm{Na}]^{2+}$} & $\mathrm{C}_{52} \mathrm{H}_{92} \mathrm{~N}_{2} \mathrm{O}_{30} \mathrm{~S}_{2} \mathrm{Na}_{2}$ & 667.2481 & 667.2508 & 4 \\
\hline
\end{tabular}




\section{ESI FAIMS HRMS Compound 2 (see text for expansion)}

FIA 2015-JULY-10 MG190A 49 (9.901) Sm (SG, 2×3.00); Cm (45:49)

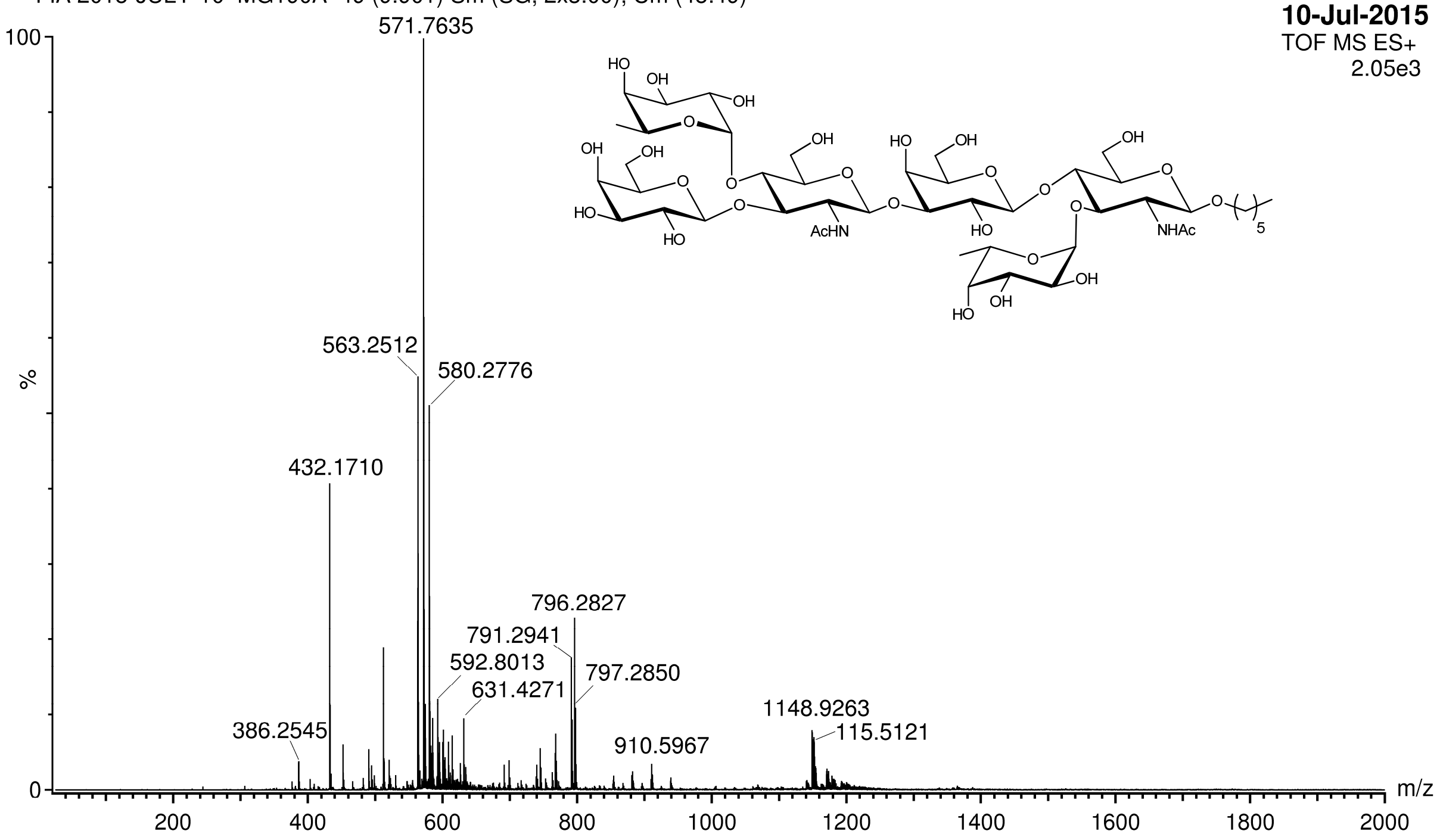




\section{ESI FAIMS HRMS Compound 3 (see text for expansion)}

FIA 2015-JULY-10 MG193A 48 (9.699) Sm (SG, 2×3.00); Cm (45:49)

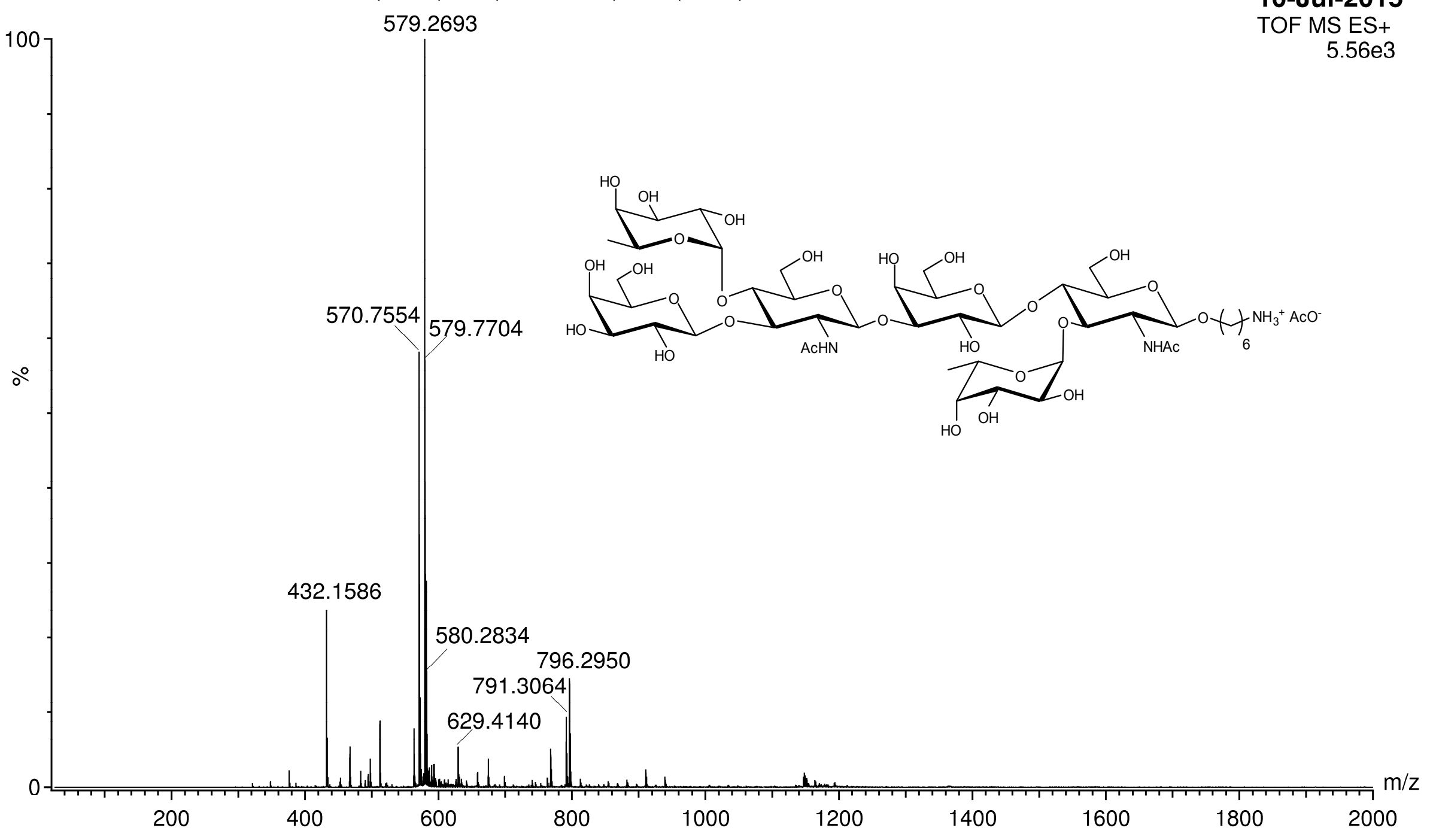




\section{ESI FAIMS HRMS Compound 4 (see text for expansion)}

JL 2014-JUNE-29 FA1 47 (9.496) Sm (SG, 2×3.00); Cm (45:49)

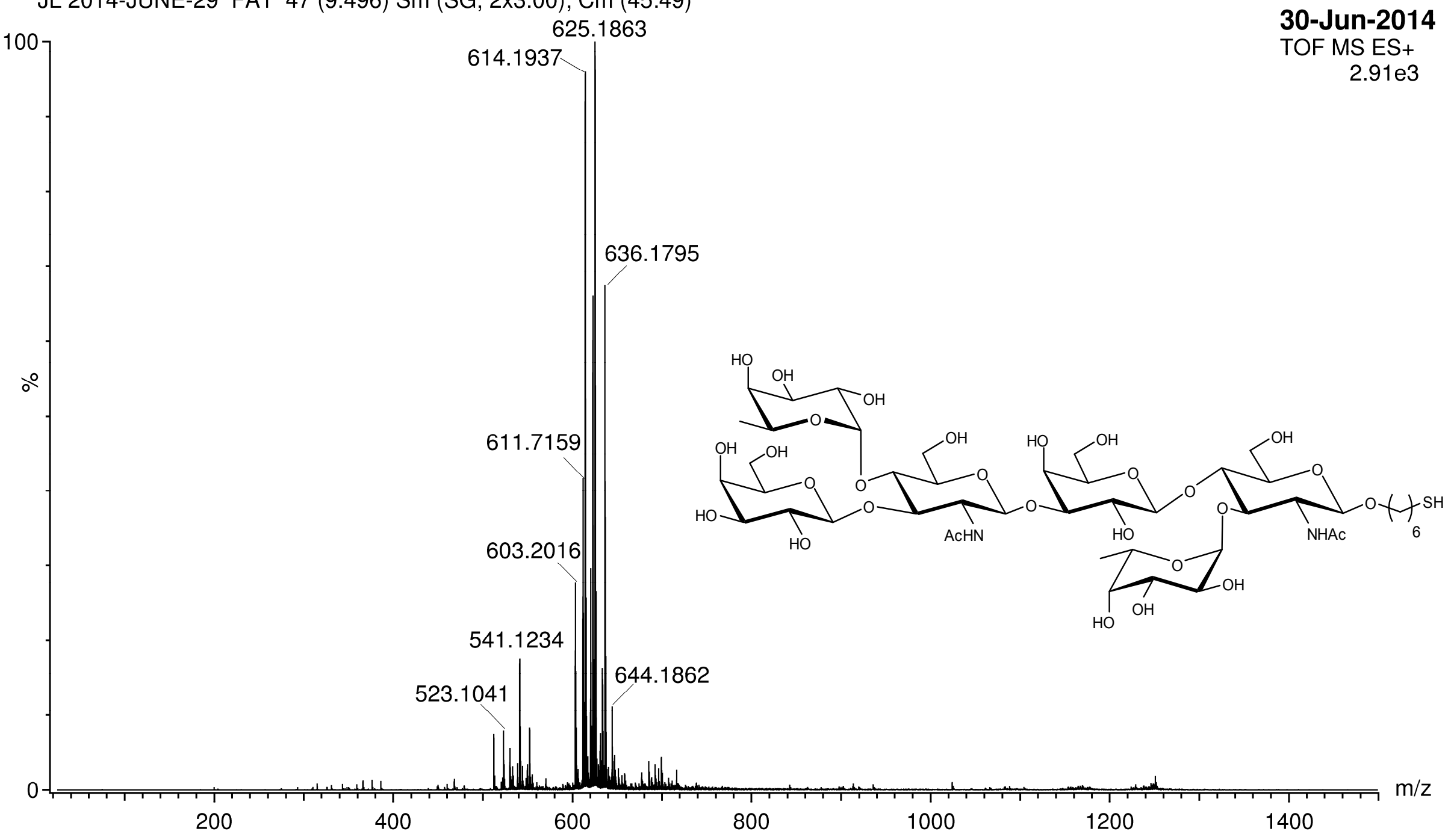




\section{ESI FAIMS HRMS Le ${ }^{x-H e x y l ~ d i s u l f i d e ~} 23$ (see text for expansion)}

FIA 2015-JULY-9 AW149A 50 (10.104) Sm (SG, 2×3.00); Cm (50:54)

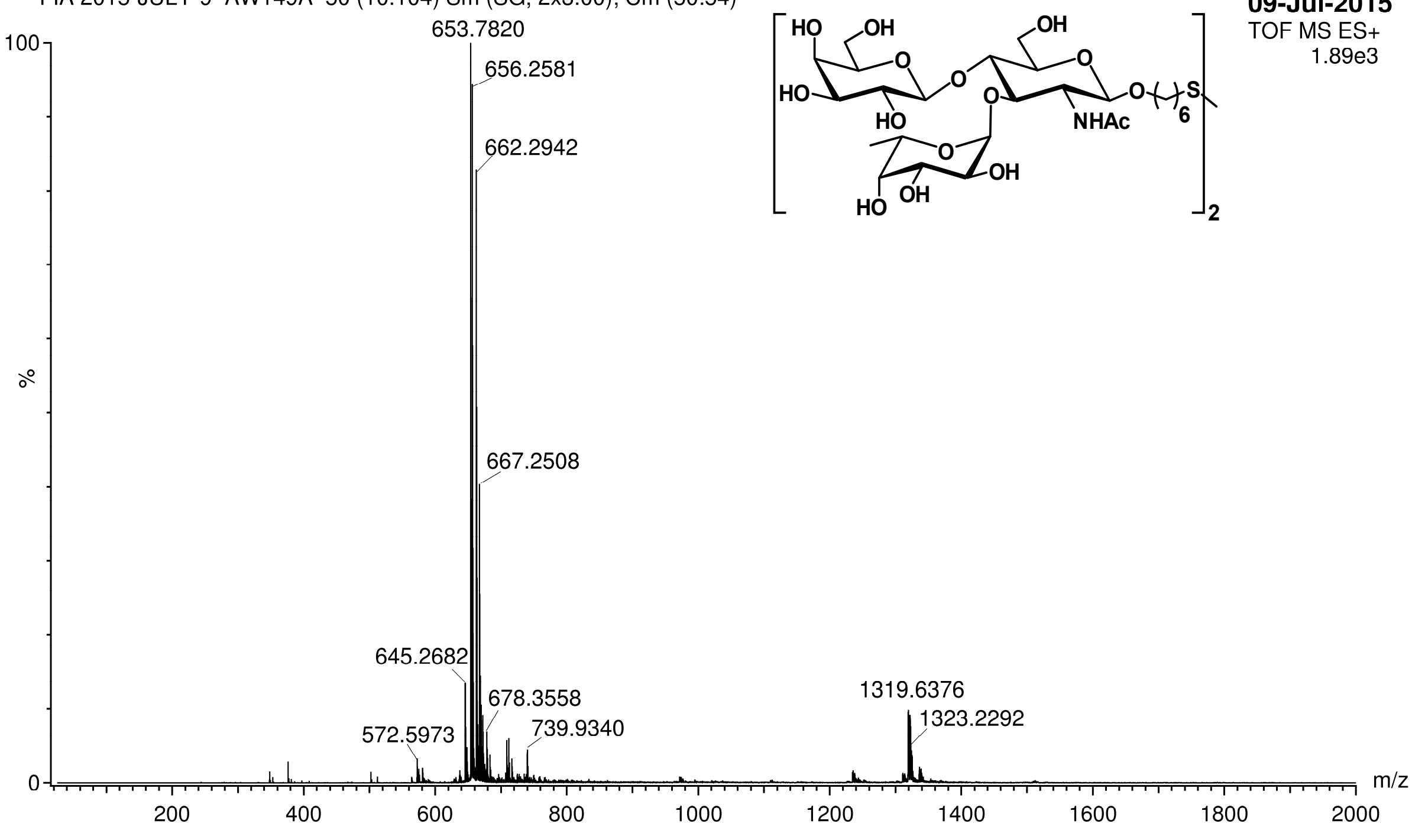




\section{ESI HRMS Compound 2}

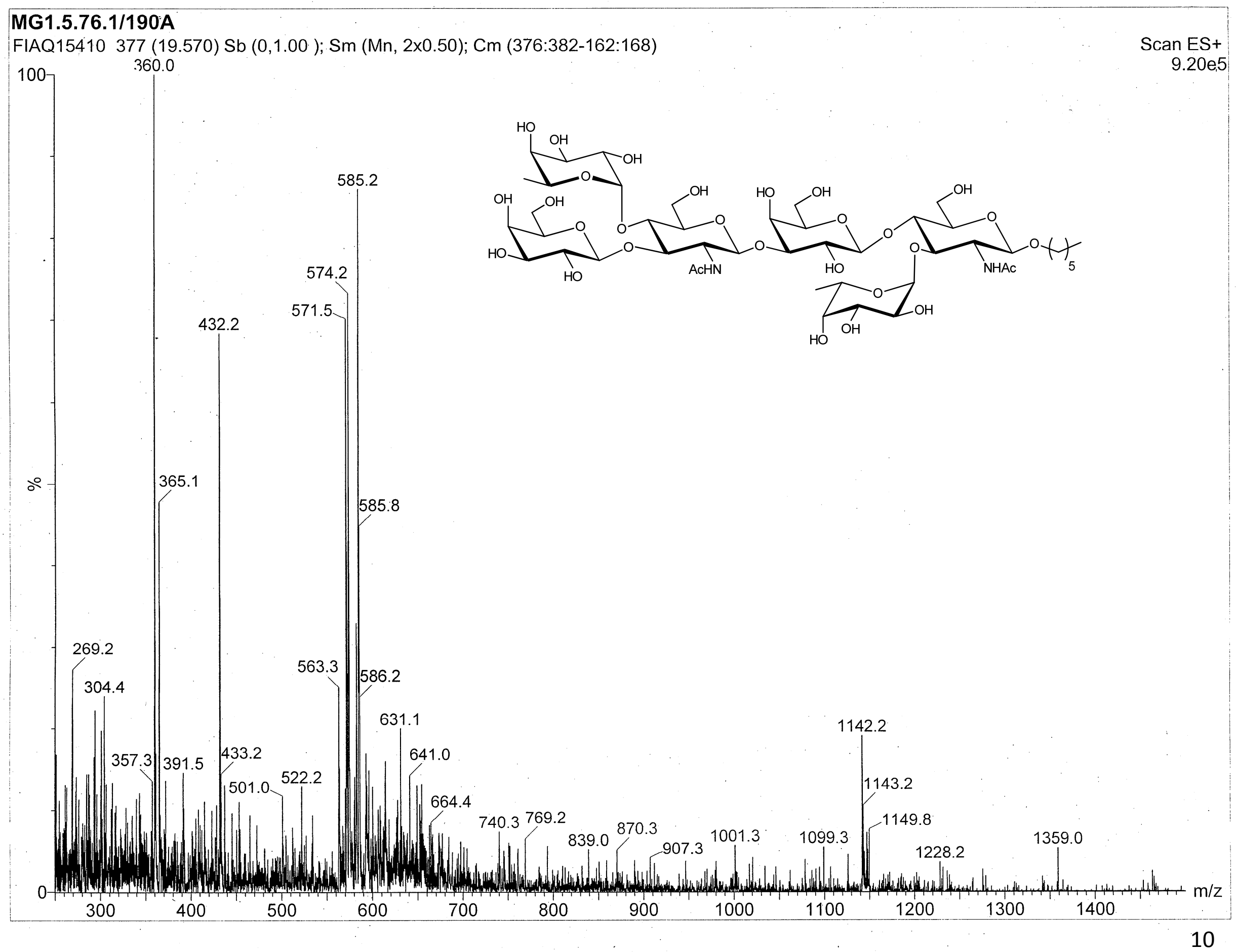




\section{ESI HRMS Compound 3}

\section{MG1.5.91.1/193A}

FIAQ15409 45 (2.343) Sb (0,1.00); Sm (Mn, 2x0.50); Cm (44:48-24:28)

100

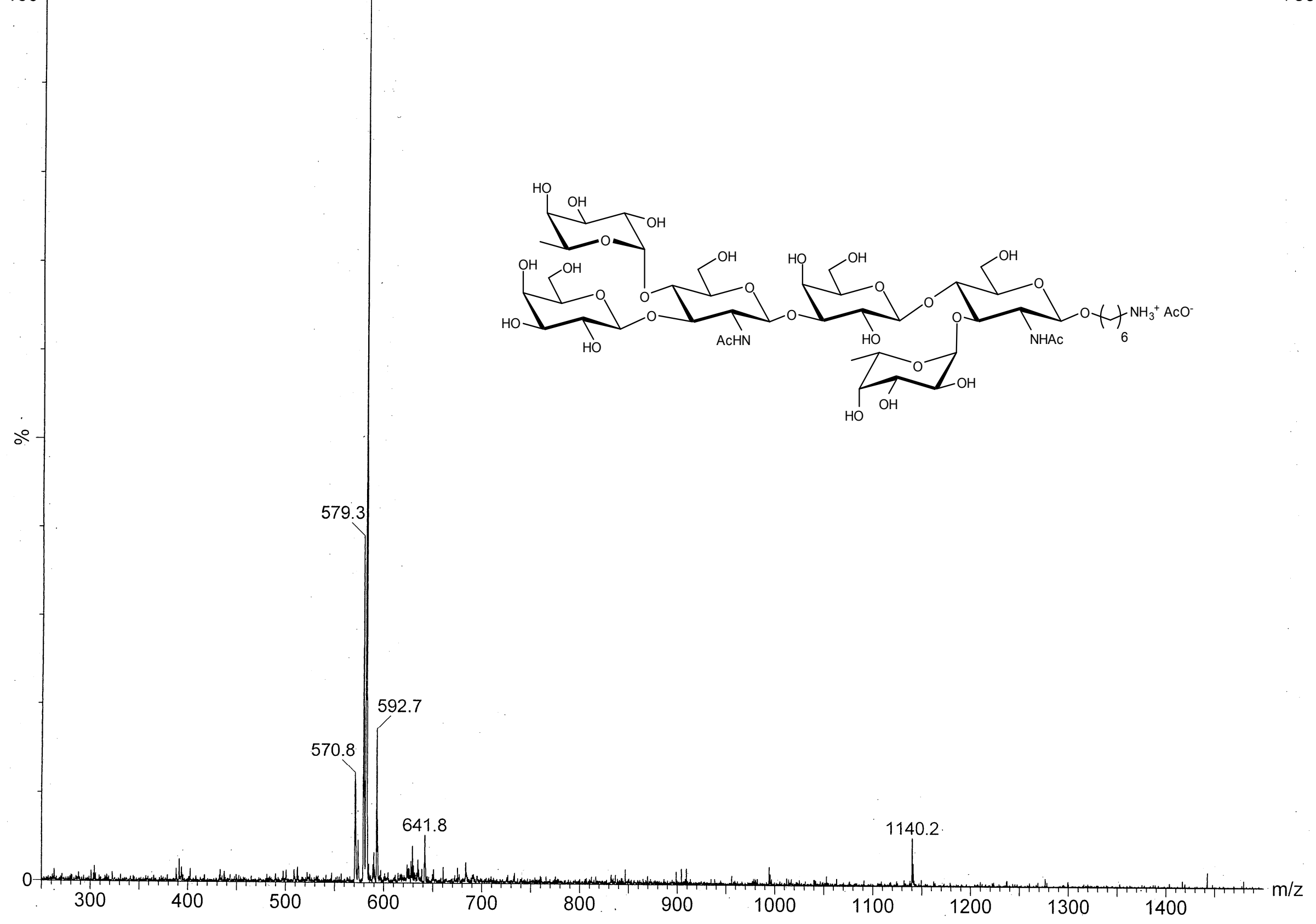




\section{ESI HRMS Compound 4 (see text for expansion)}

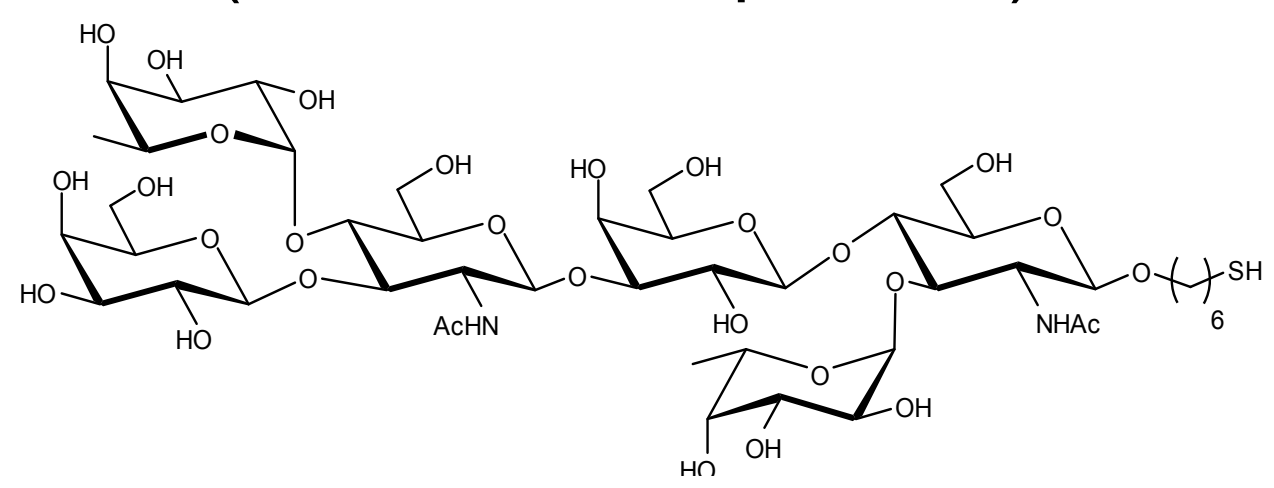

MG.1.5.98.1/195A

FIAB15594 503 (9.588) AM (Cen,6, 80.00, Ht,10000.0,622.03,1.00); Sb (99,10.00); Sm (Mn, 3×3.00); Cm (502:508)

${ }^{100}$
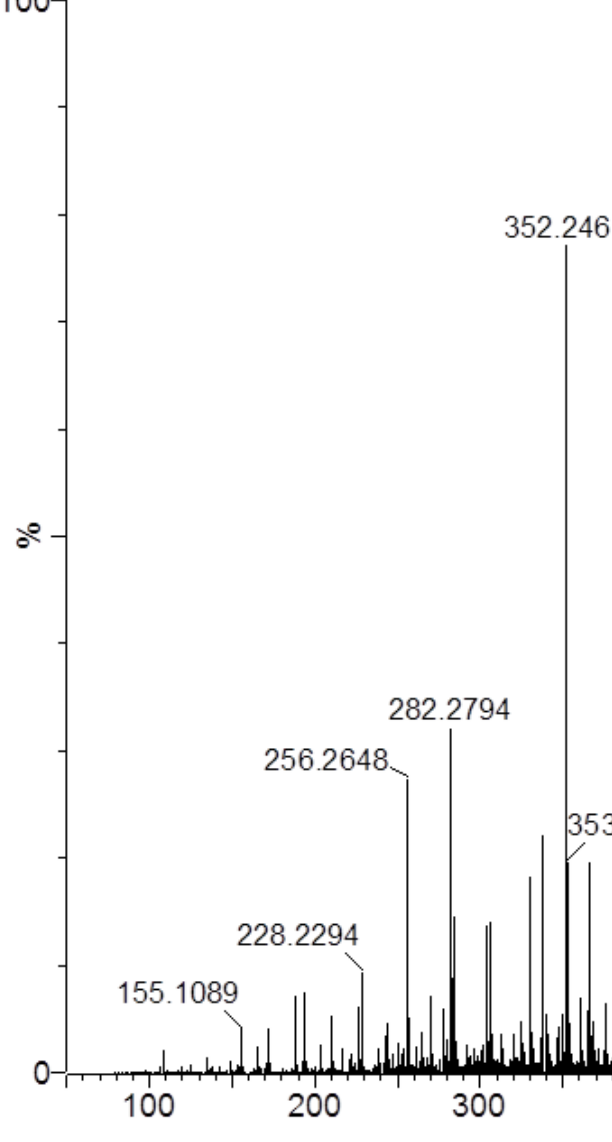

\subsection{1}
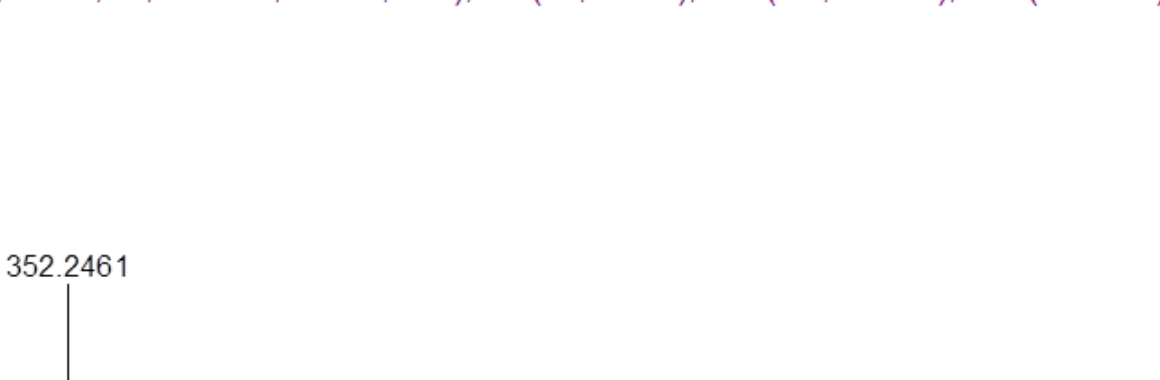


\section{General Experimental Procedures}

${ }^{1} \mathrm{H}$ NMR (600.13 or $400.13 \mathrm{MHz}$ ) and ${ }^{13} \mathrm{C}$ NMR (150.9 or $100.6 \mathrm{MHz}$ ) spectra were recorded for compounds solubilized in $\mathrm{CDCl}_{3}$ (internal standard, for ${ }^{1} \mathrm{H}$ residual $\mathrm{CHCl}_{3} \delta_{\mathrm{H}} 7.24$; for ${ }^{13} \mathrm{C} \mathrm{CDCl}_{3} \delta_{\mathrm{C}} 77.0$ ), $\mathrm{CD}_{3} \mathrm{OD}$ (internal standard, for ${ }^{1} \mathrm{H}$ residual $\mathrm{CD}_{2} \mathrm{HOD} \delta_{\mathrm{H}} 3.30$; for ${ }^{13} \mathrm{C} \mathrm{CD} \mathrm{CD}$ $\delta_{\mathrm{C}} 49.0$ ) or $\mathrm{D}_{2} \mathrm{O}$ [external standard, for ${ }^{1} \mathrm{H}$ (sodium 2,2-dimethyl-2-silapentane-5-sulfonate (DSS) $\delta_{\mathrm{H}} 0.00$; for ${ }^{13} \mathrm{C} \delta_{\mathrm{H}} 0.0$ )]. ${ }^{1} \mathrm{H}$ NMR and ${ }^{13} \mathrm{C}$ NMR chemical shifts are reported in part per million (ppm). Coupling constants $(J)$ were obtained from a first-order analysis of onedimensional spectra and are reported in hertz $(\mathrm{Hz})$. Assignments of proton and carbon resonances were based on two dimensional ${ }^{1} \mathrm{H}-{ }^{1} \mathrm{H}$ COSY and ${ }^{13} \mathrm{C}-1$ H HSQC correlation experiments as well as $1 \mathrm{D}$ TOCSY experiments. ${ }^{1} \mathrm{H}$ NMR data are reported using standard abbreviations: singlet (s), doublet (d), triplet (t), doublet of doublet (dd), doublet of doublet of doublet (ddd), triplet of doublet (td), broad signal (bs) and multiplet (m). Analytical thin-layer chromatography (TLC) was performed on aluminum plates precoated with Silica Gel 60 $\mathrm{F}_{254}(250 \mu \mathrm{m})$ containing a fluorescent indicator. The plates were visualized under $\mathrm{UV}$ and/or charred with a $20 \%$ solution of $\mathrm{H}_{2} \mathrm{SO}_{4}$ in $\mathrm{EtOH}$. Compounds were purified by flash chromatography with Silica Gel 60 (230-400 mesh) unless otherwise stated. Solvents were distilled and dried according to standard procedures and organic solutions were dried over $\mathrm{Na}_{2} \mathrm{SO}_{4}$ and concentrated under reduced pressure. Molecular sieves were activated by heating at $300{ }^{\circ} \mathrm{C}$ overnight under reduced pressure over $\mathrm{P}_{2} \mathrm{O}_{5}$. Zinc powder $(2 \mathrm{~g})$ was activated by successive washings with $\mathrm{HCl} 2 \mathrm{~N}(25 \mathrm{~mL}), \mathrm{H}_{2} \mathrm{O}(50 \mathrm{~mL}), \mathrm{MeOH}(50 \mathrm{~mL})$ and $\mathrm{Et}_{2} \mathrm{O}(50 \mathrm{~mL})$ on a fritted glass. The sonication reactions were carried out in a thermostated $\left(50 \pm 4{ }^{\circ} \mathrm{C}\right)$ ultrasonic cleaning bath at $40 \mathrm{kHz}$ and involved the partial immersion of the reaction vessel (10 mL roundbottomed flask equipped with a sealed glass cap) into the water tank (dimensions were $292 \times 152 \times 152$ mm with a liquid holding capacity of 5.71 L). Reverse-phase HPLC purifications were carried out on Prep Nova Pak ${ }^{\circledR}$ HR C18, $6 \mu \mathrm{m} 60 \AA$ columns using mixtures of HPLC grade acetonitrile and milli-Q water. Purifications by gel permeation chromatography were carried out on a Biogel P2 column $(100 \times 1 \mathrm{~cm})$ eluted with water or $0.05 \mathrm{M}$ aq AcONH$_{4}$. Optical rotations were measured at $22{ }^{\circ} \mathrm{C}$ and are reported as follows: $[\alpha]_{\mathrm{D}}(c$ in grams per $100 \mathrm{~mL}$, solvent). Mass spectra were obtained under electrospray ionization (ESI) on a high resolution mass spectrometer equipped with a TOF analyzer. 


\section{Compound 10, $400 \mathrm{MHz}, 297 \mathrm{~K}, \mathrm{CDCl}_{3}$}

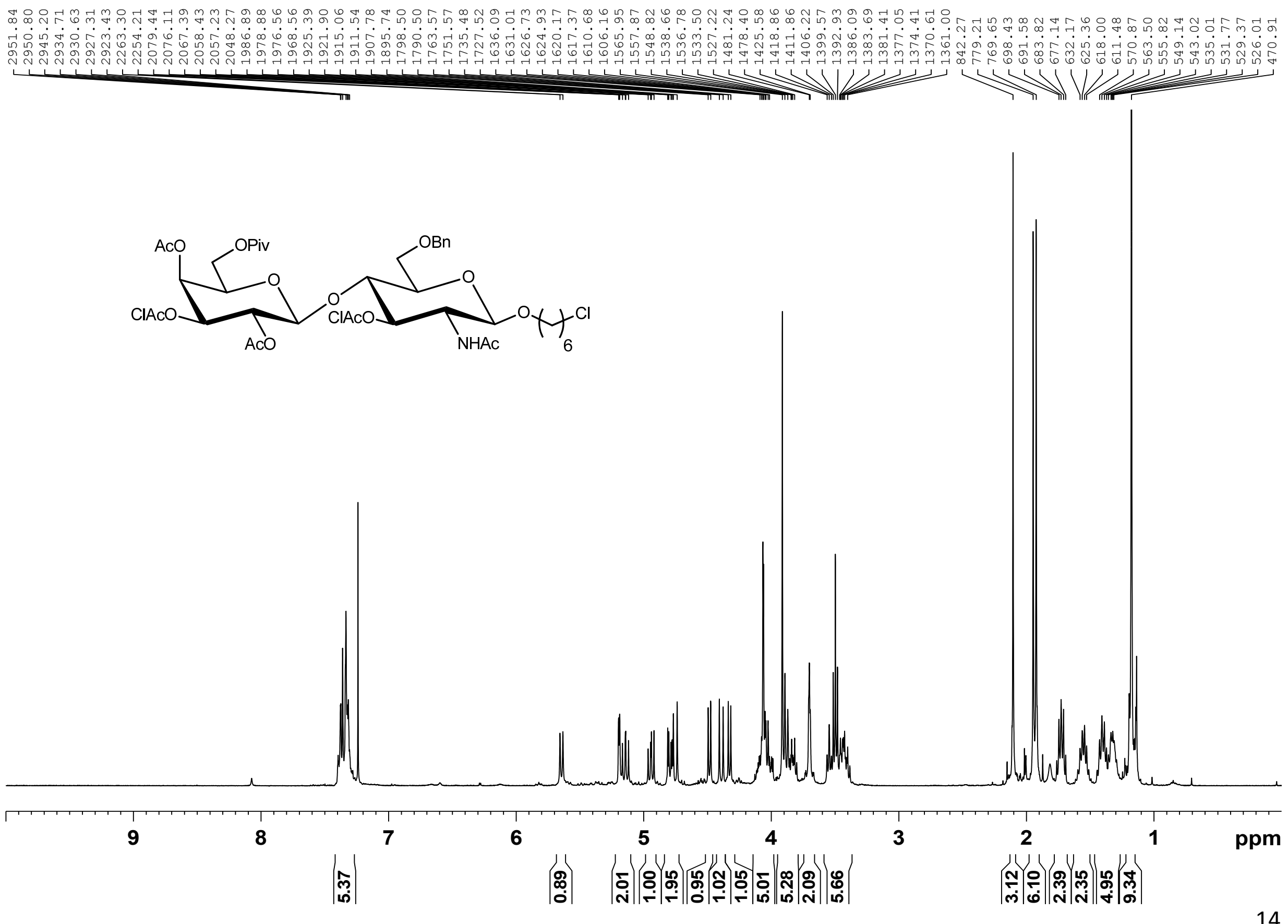




\section{Compound 10, $100 \mathrm{MHz}, 297 \mathrm{~K}, \mathrm{CDCl}_{3}$}

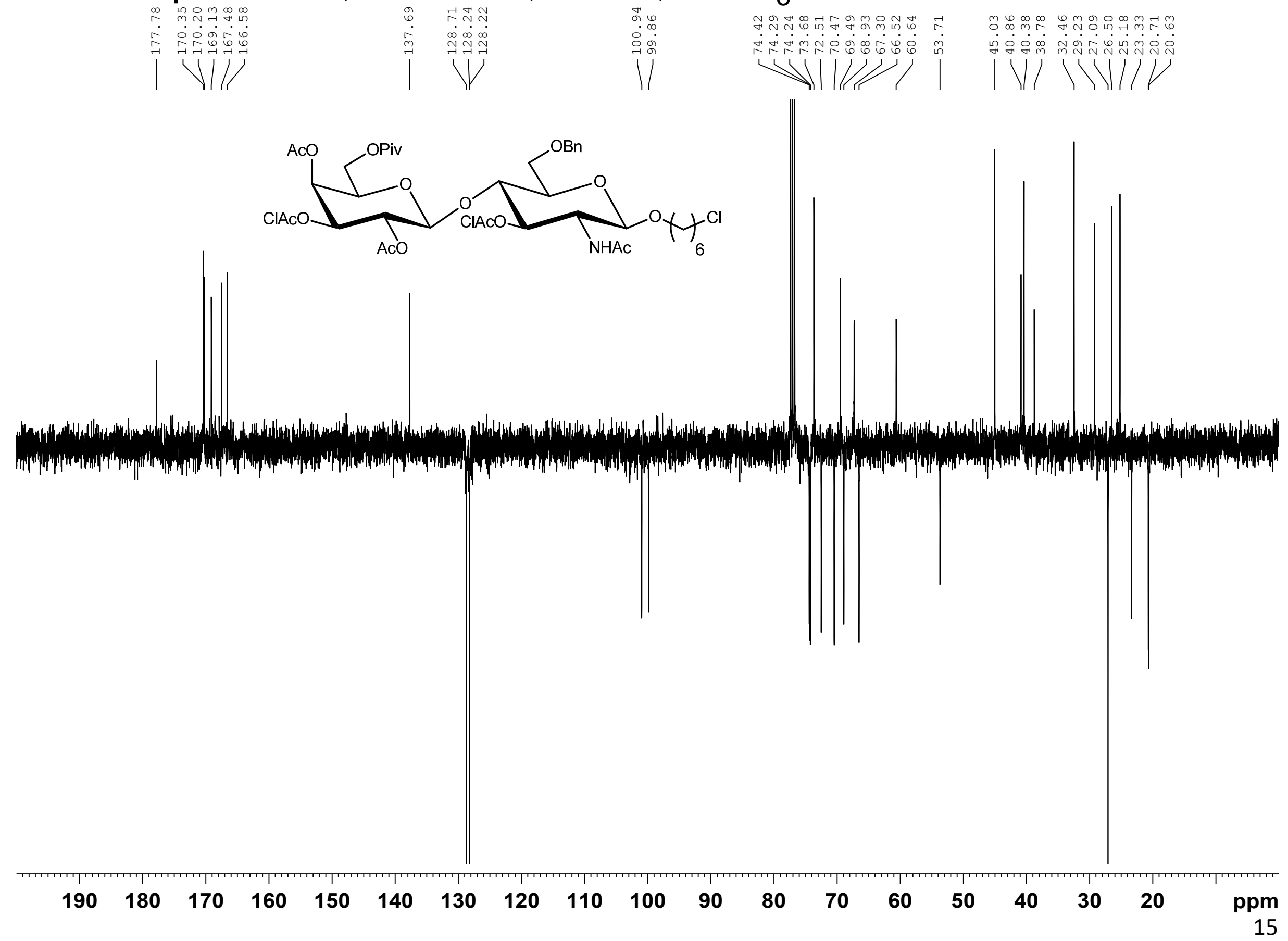


Compound 11, $400 \mathrm{MHz}, 296 \mathrm{~K}, \mathrm{CDCl}_{3}$

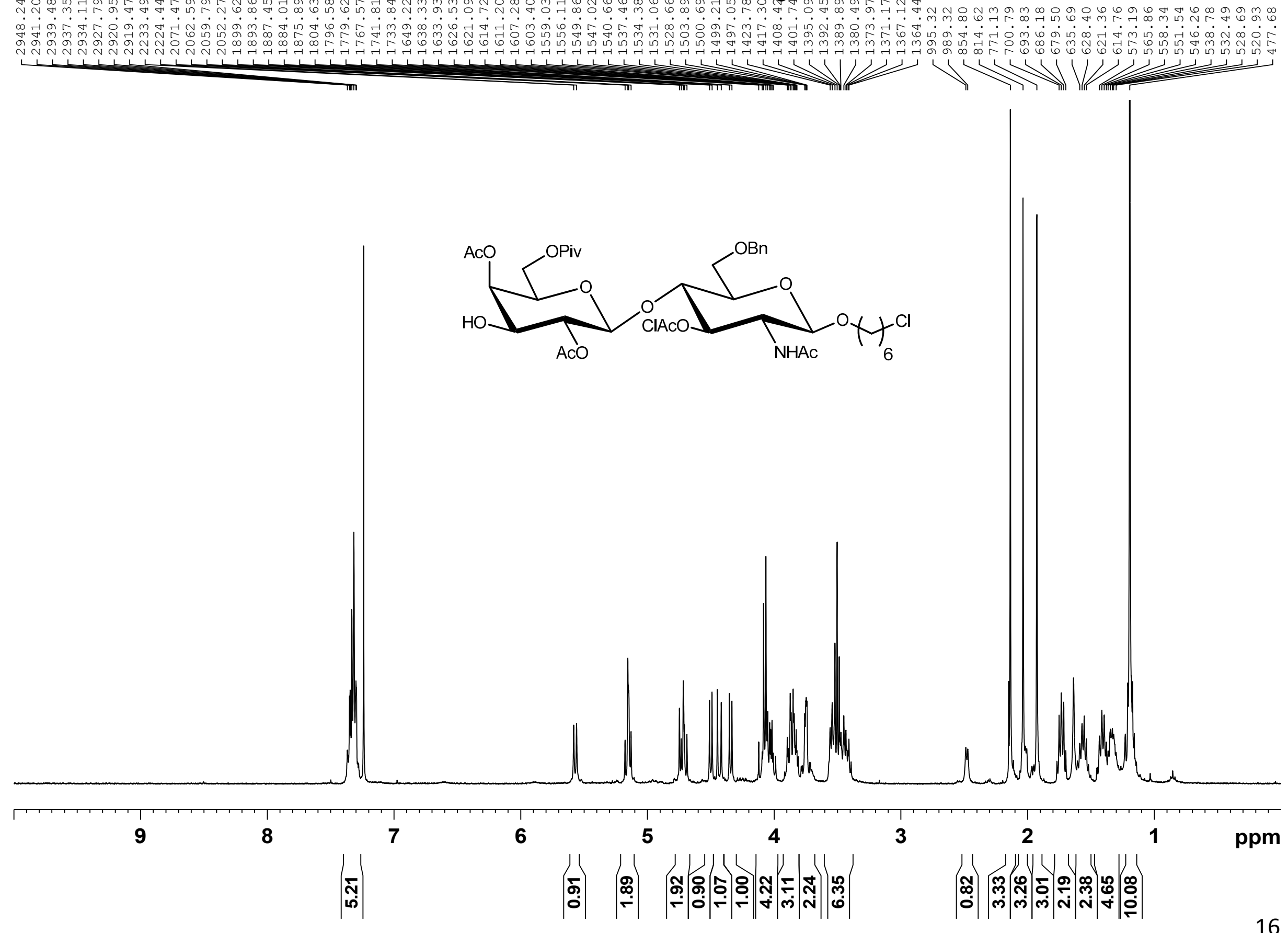


Compound 11, $100 \mathrm{MHz}, 296 \mathrm{~K}, \mathrm{CDCl}_{3}$

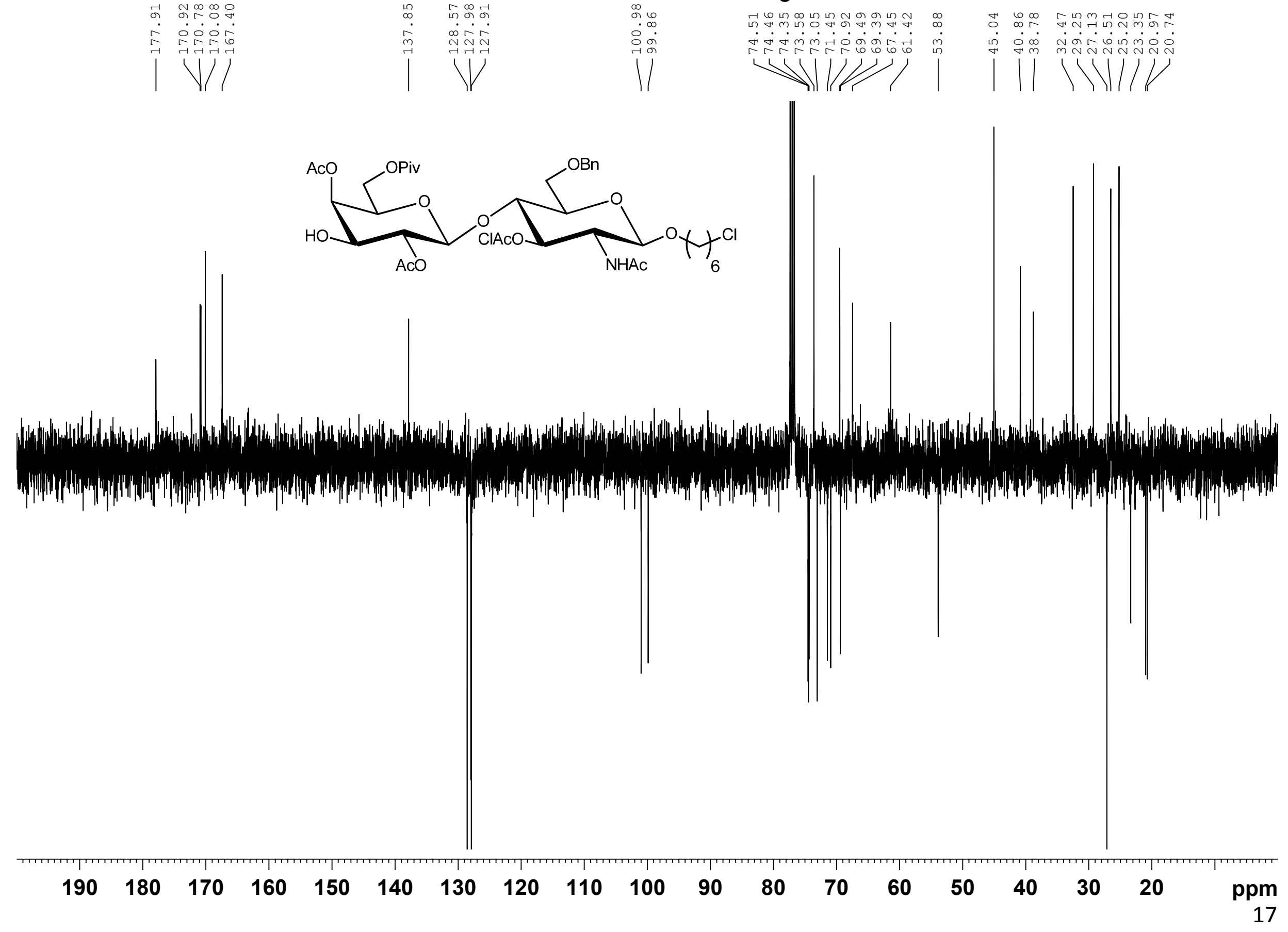


Compound 12, $600 \mathrm{MHz}, 295 \mathrm{~K}, \mathrm{CDCl}_{3}$

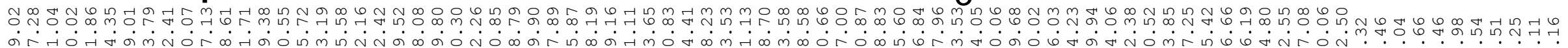

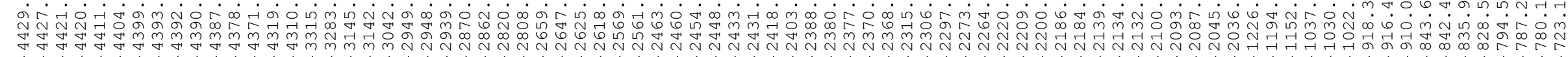
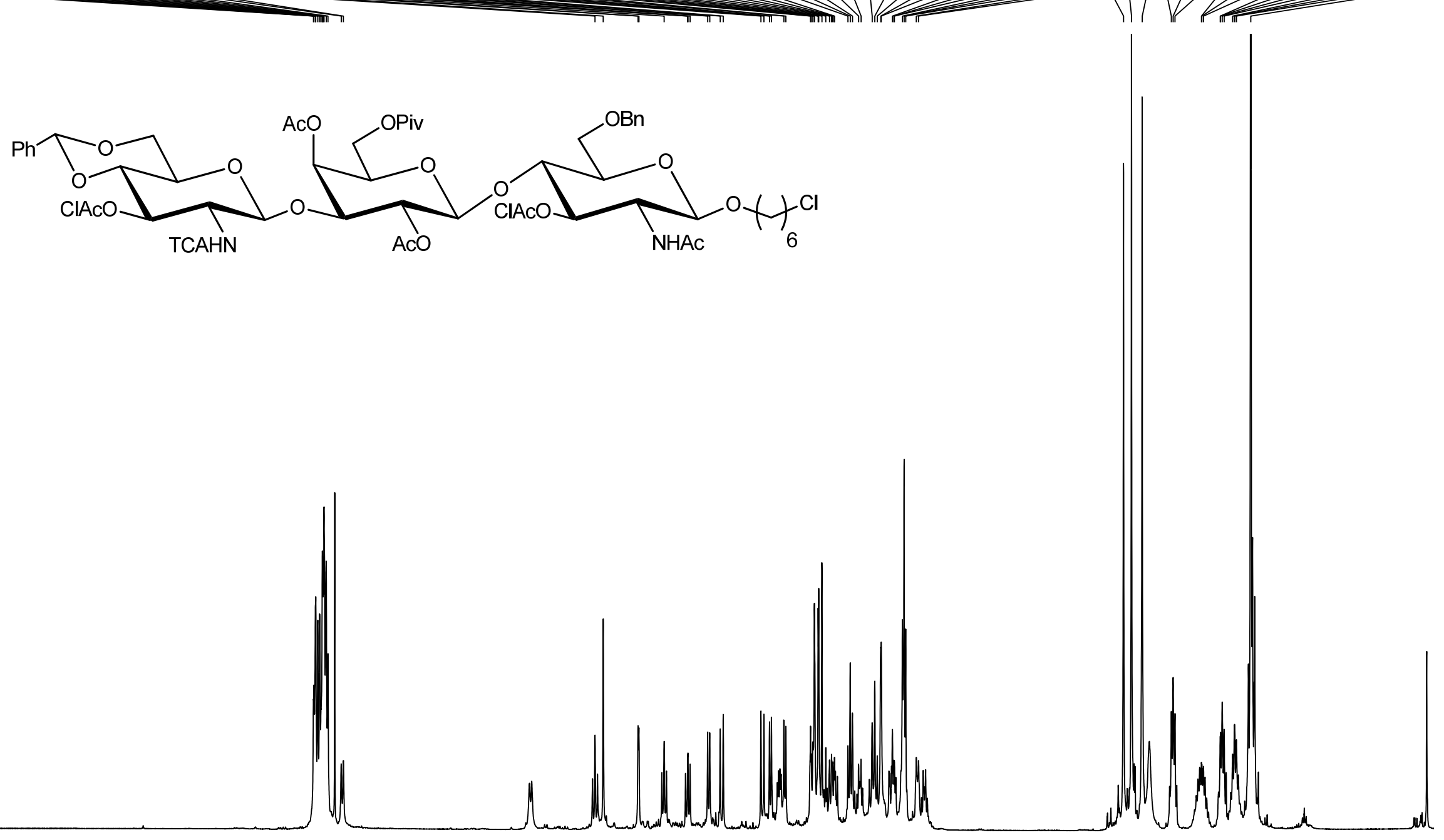

9

8

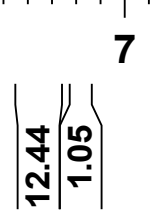

5

4

(j)

3

2

중

N่ 
Compound 12, $600 \mathrm{MHz}, 295 \mathrm{~K}, \mathrm{CDCl}_{3}$

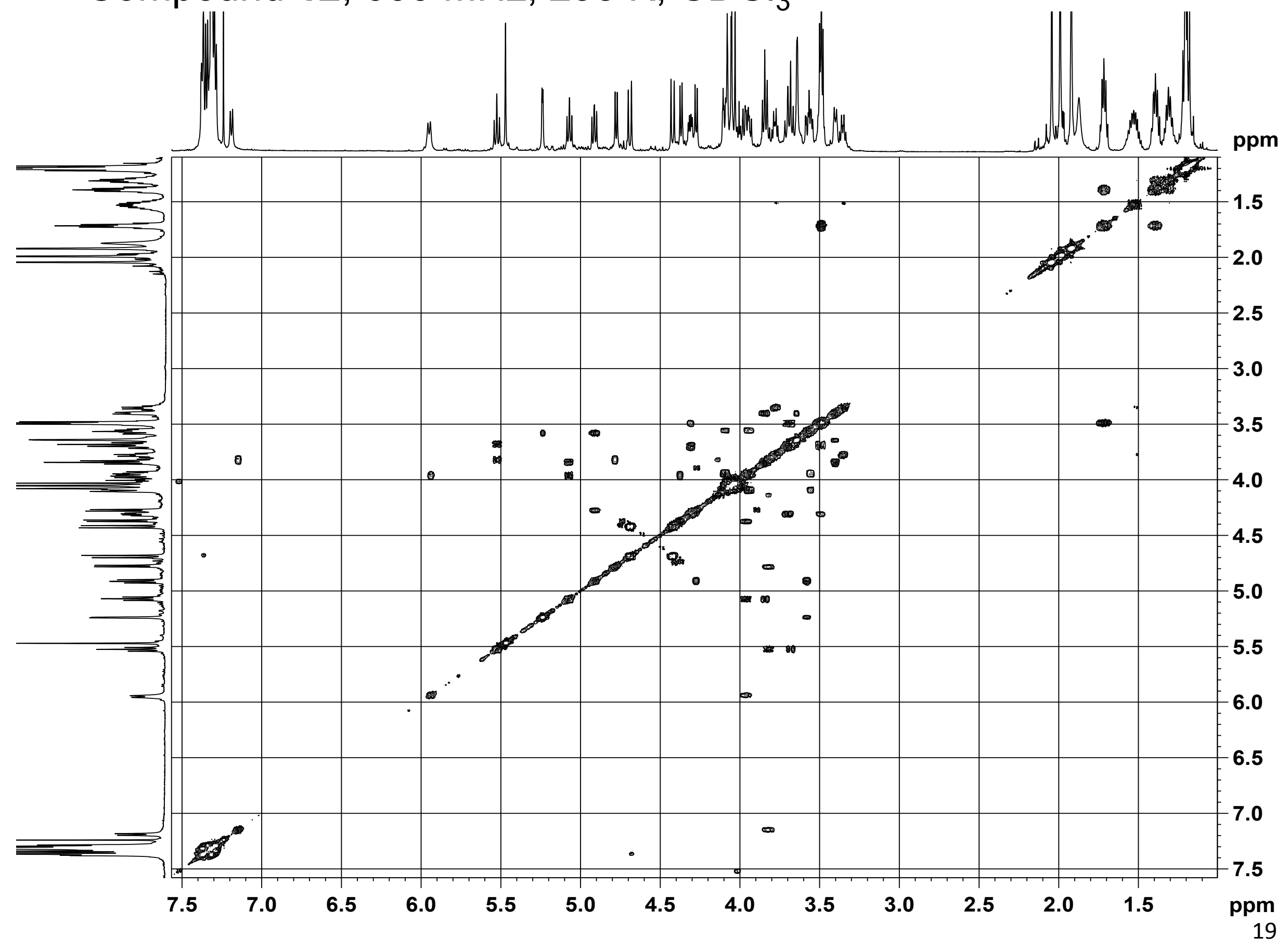


Compound 12, $125 \mathrm{MHz}, 295 \mathrm{~K}, \mathrm{CDCl}_{3}$

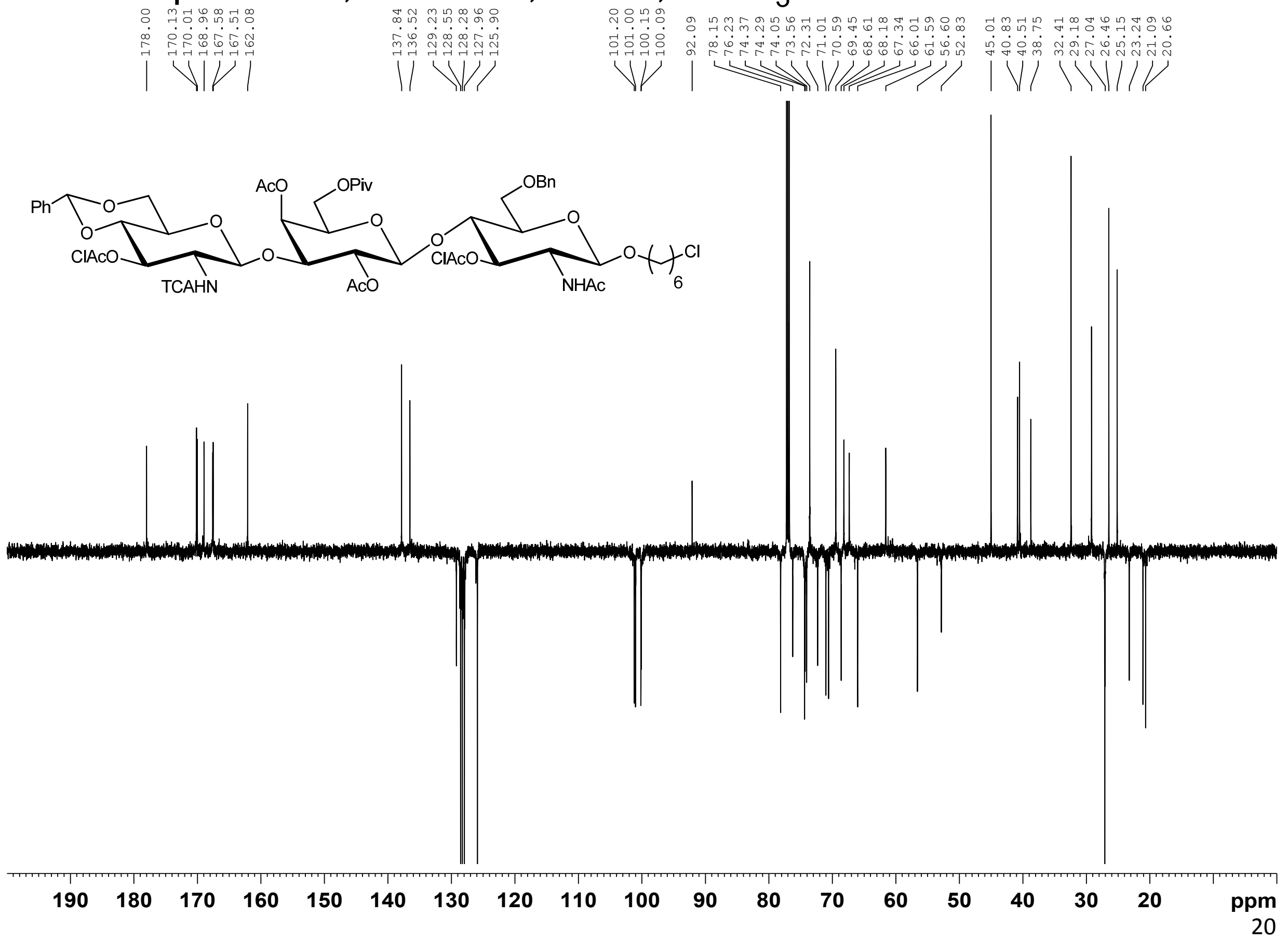


Compound 12, $125 \mathrm{MHz}, 295 \mathrm{~K}, \mathrm{CDCl}_{3}$

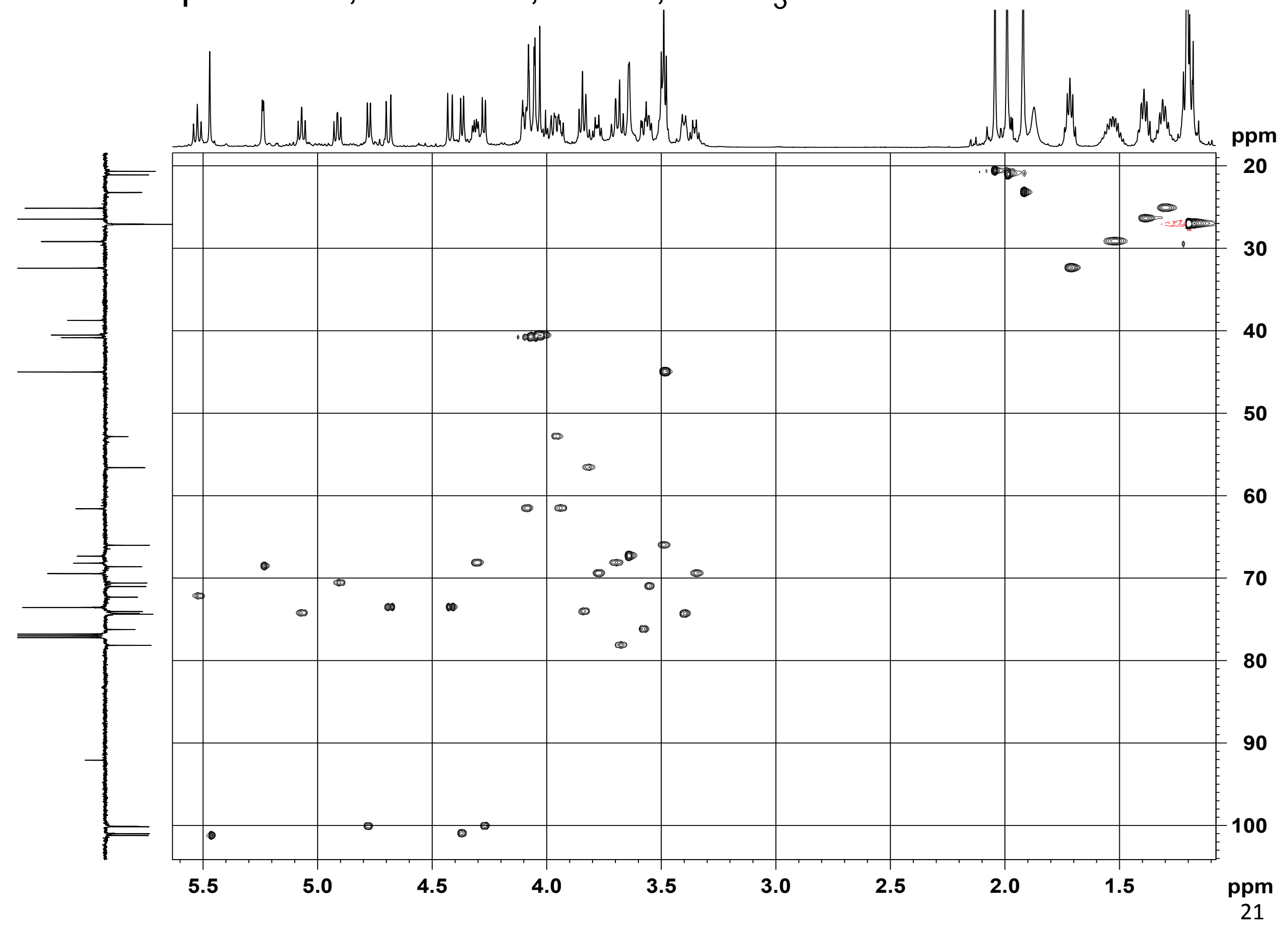




\section{Compound 13, $600 \mathrm{MHz}, 296 \mathrm{~K}, \mathrm{CDCl}_{3}$}

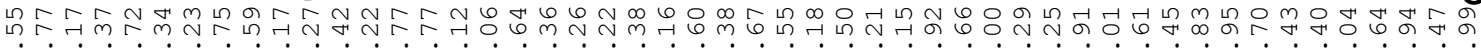

Tाilा
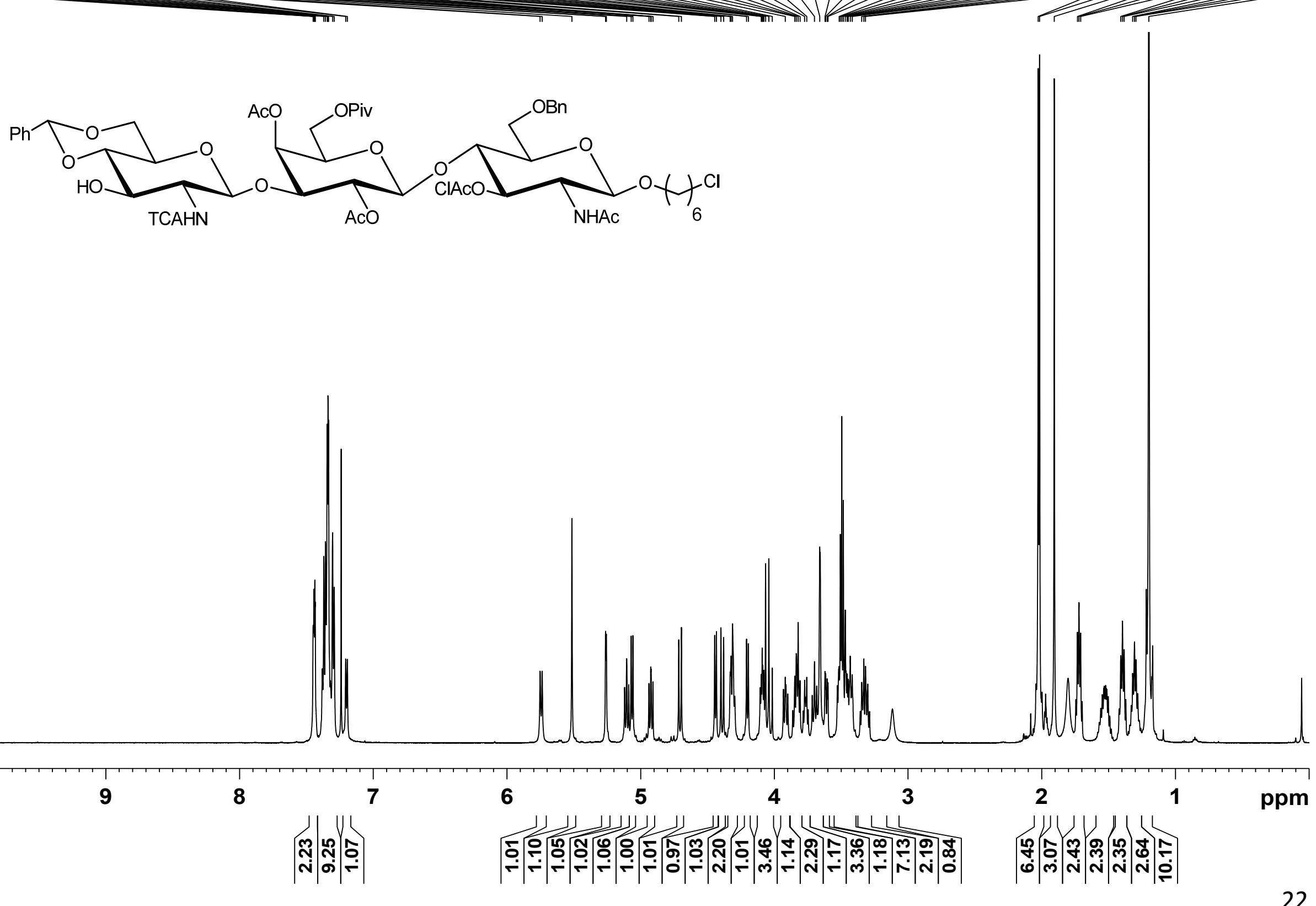
Compound 13, $600 \mathrm{MHz}, 296 \mathrm{~K}, \mathrm{CDCl}_{3}$

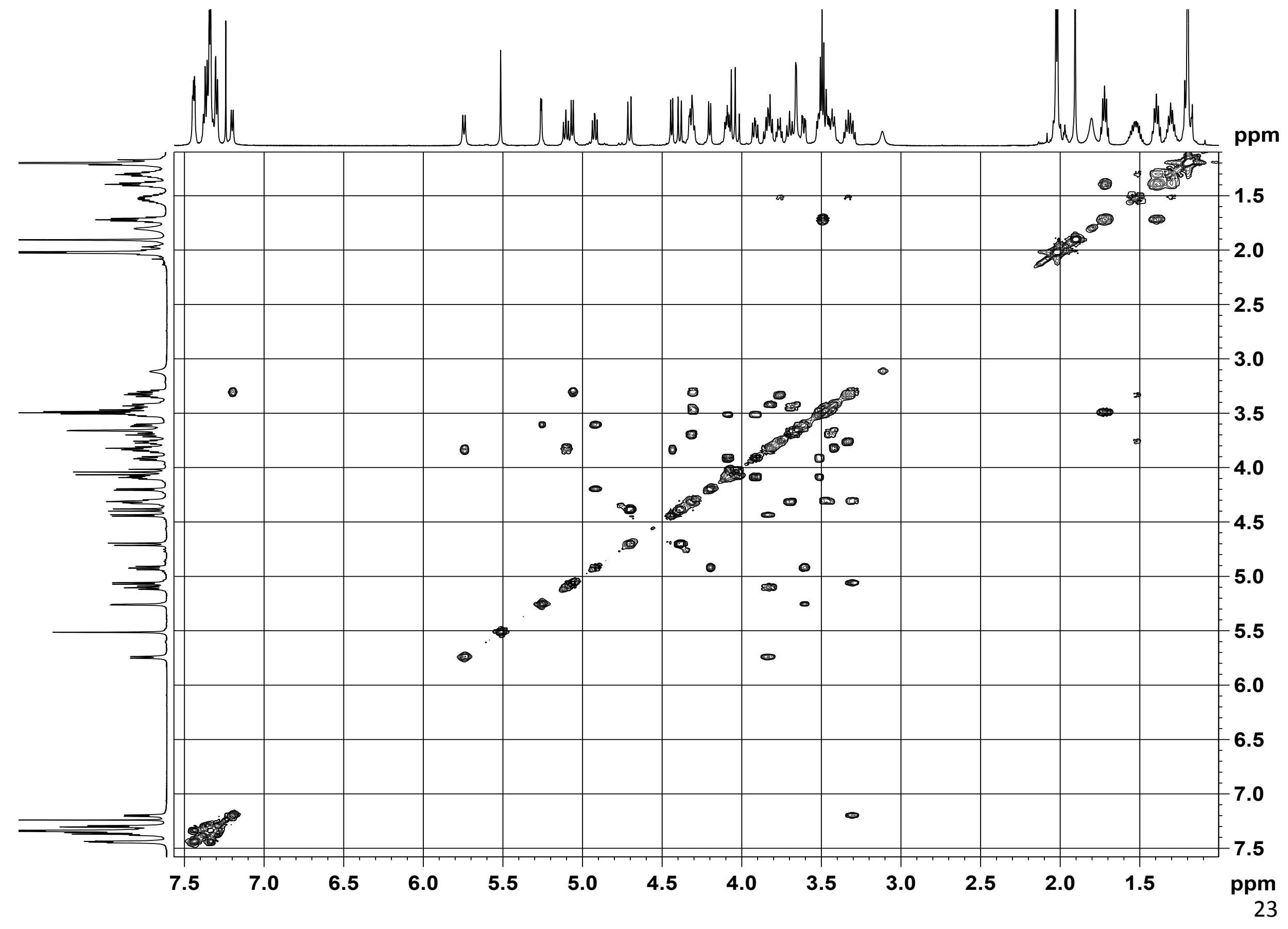


Compound 13, $125 \mathrm{MHz}, 296 \mathrm{~K}, \mathrm{CDCl}_{3}$

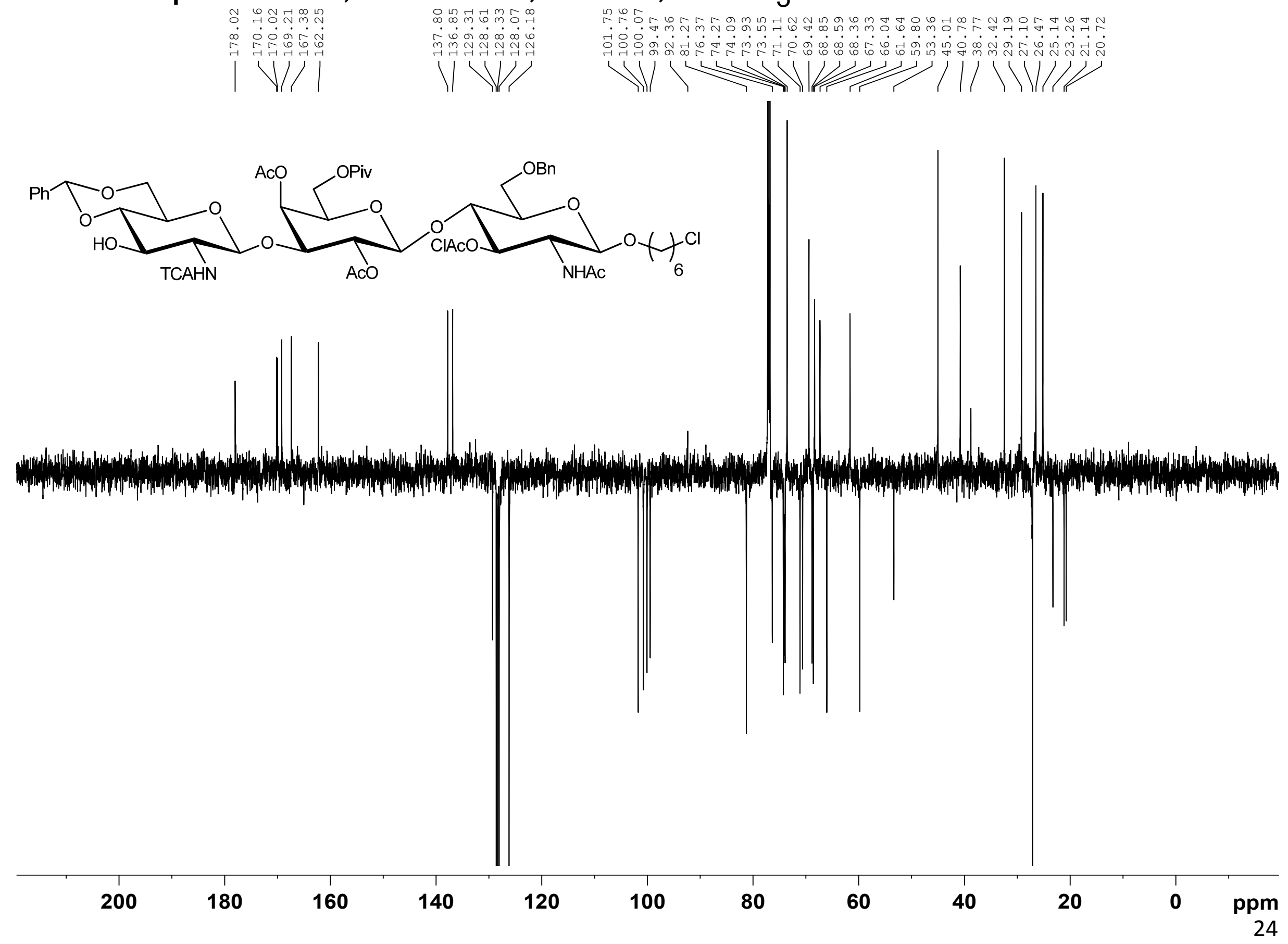


Compound 13, $125 \mathrm{MHz}, 296 \mathrm{~K}, \mathrm{CDCl}_{3}$

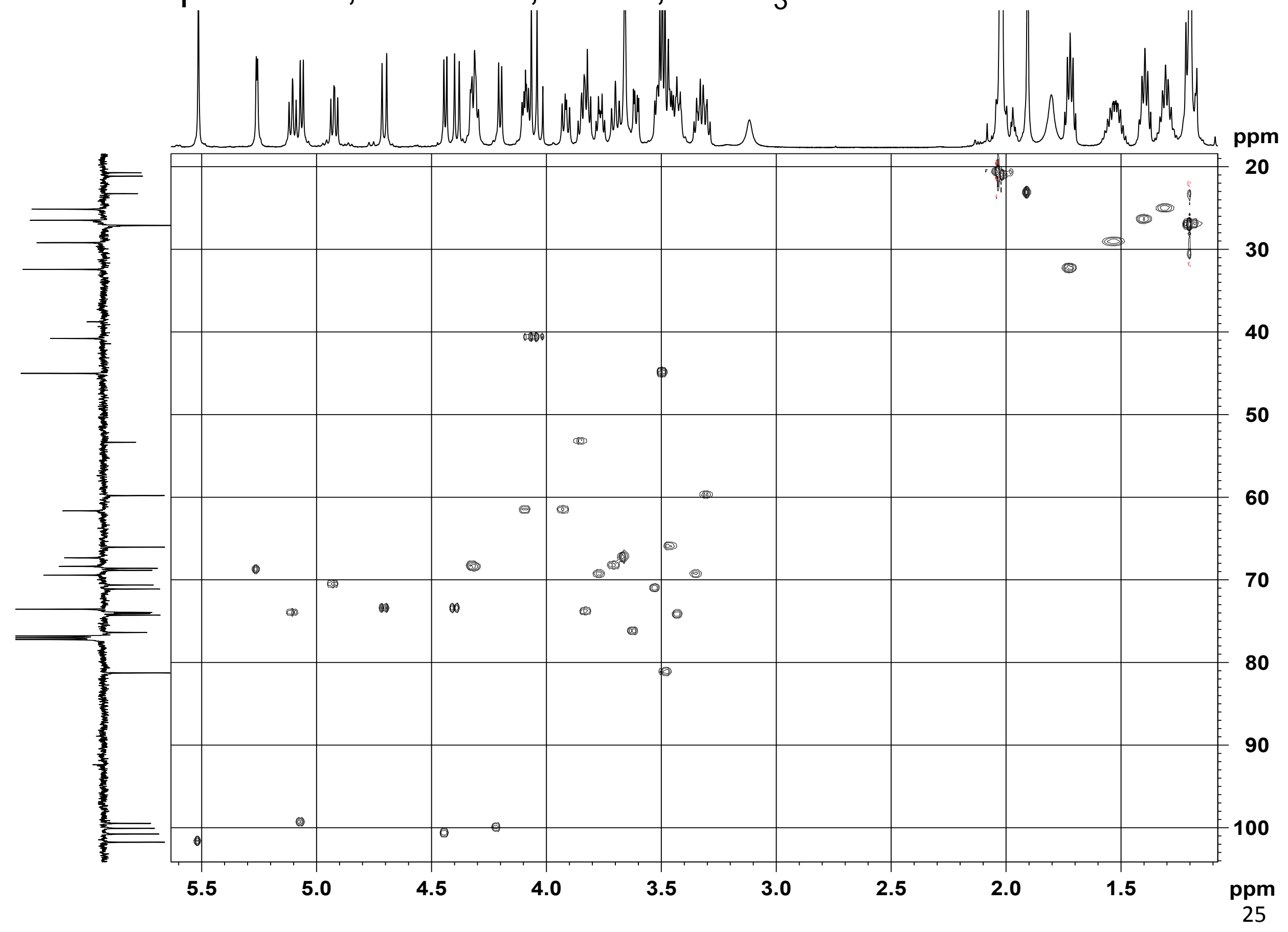




\section{Compound 14, $600 \mathrm{MHz}, 296 \mathrm{~K}, \mathrm{CDCl}_{3}$}

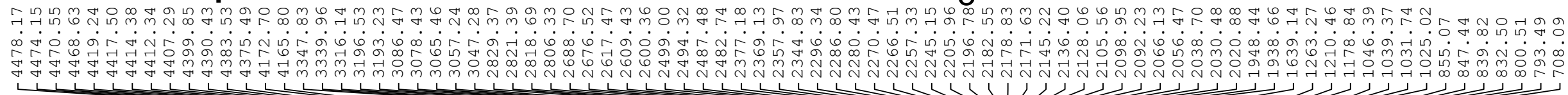

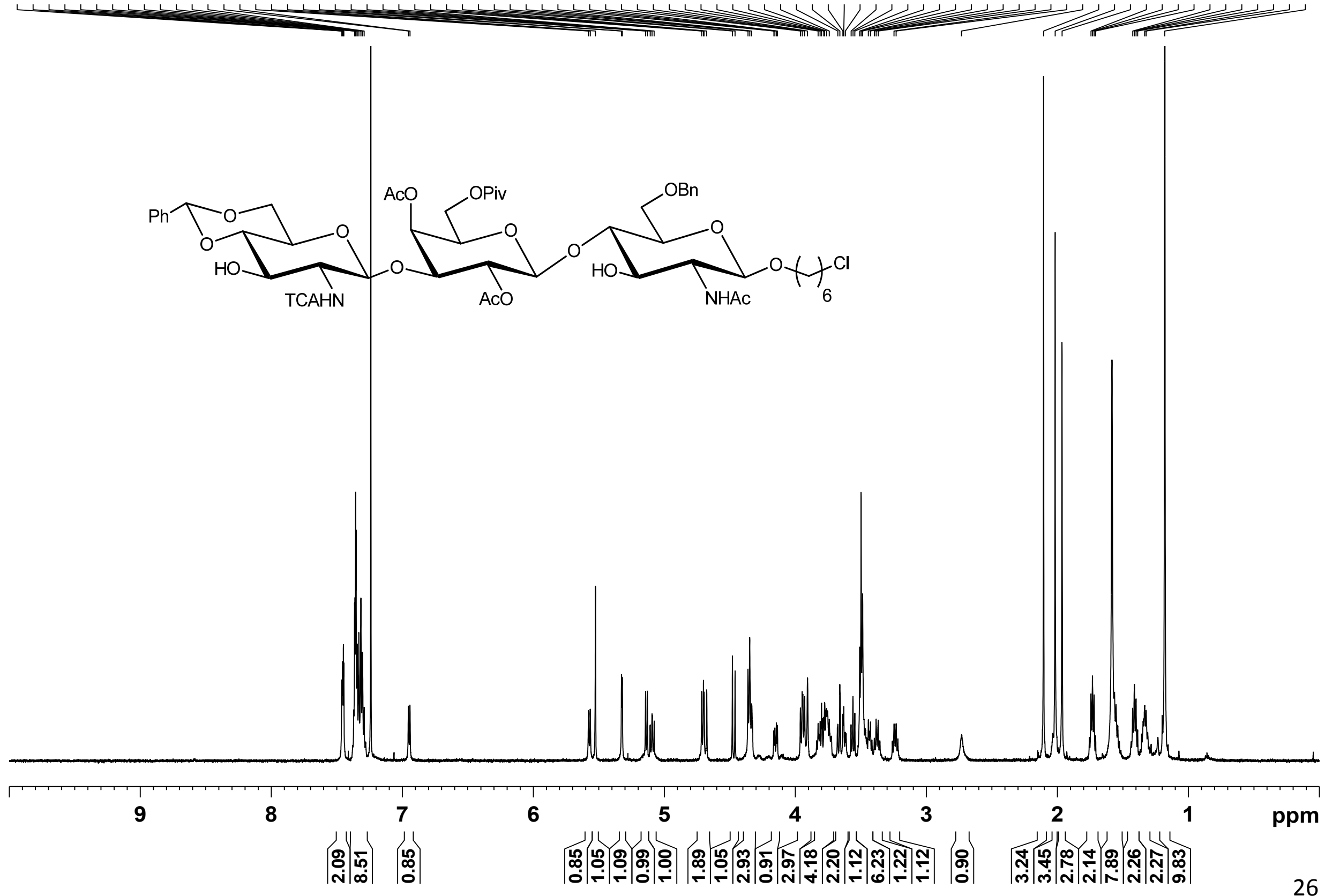


Compound 14, $600 \mathrm{MHz}, 296 \mathrm{~K}, \mathrm{CDCl}_{3}$

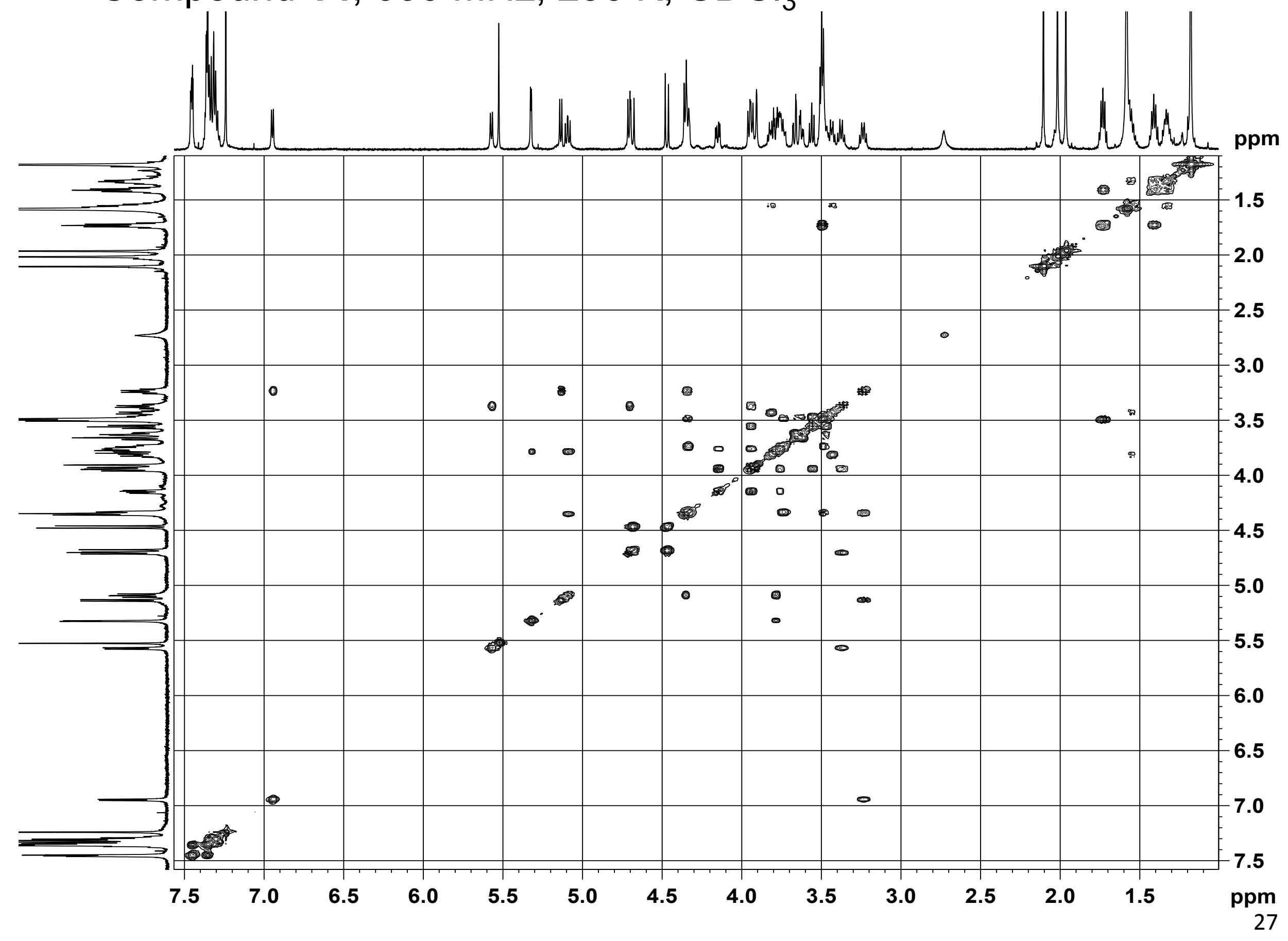


Compound 14, $125 \mathrm{MHz}, 296 \mathrm{~K}, \mathrm{CDCl}_{3}$

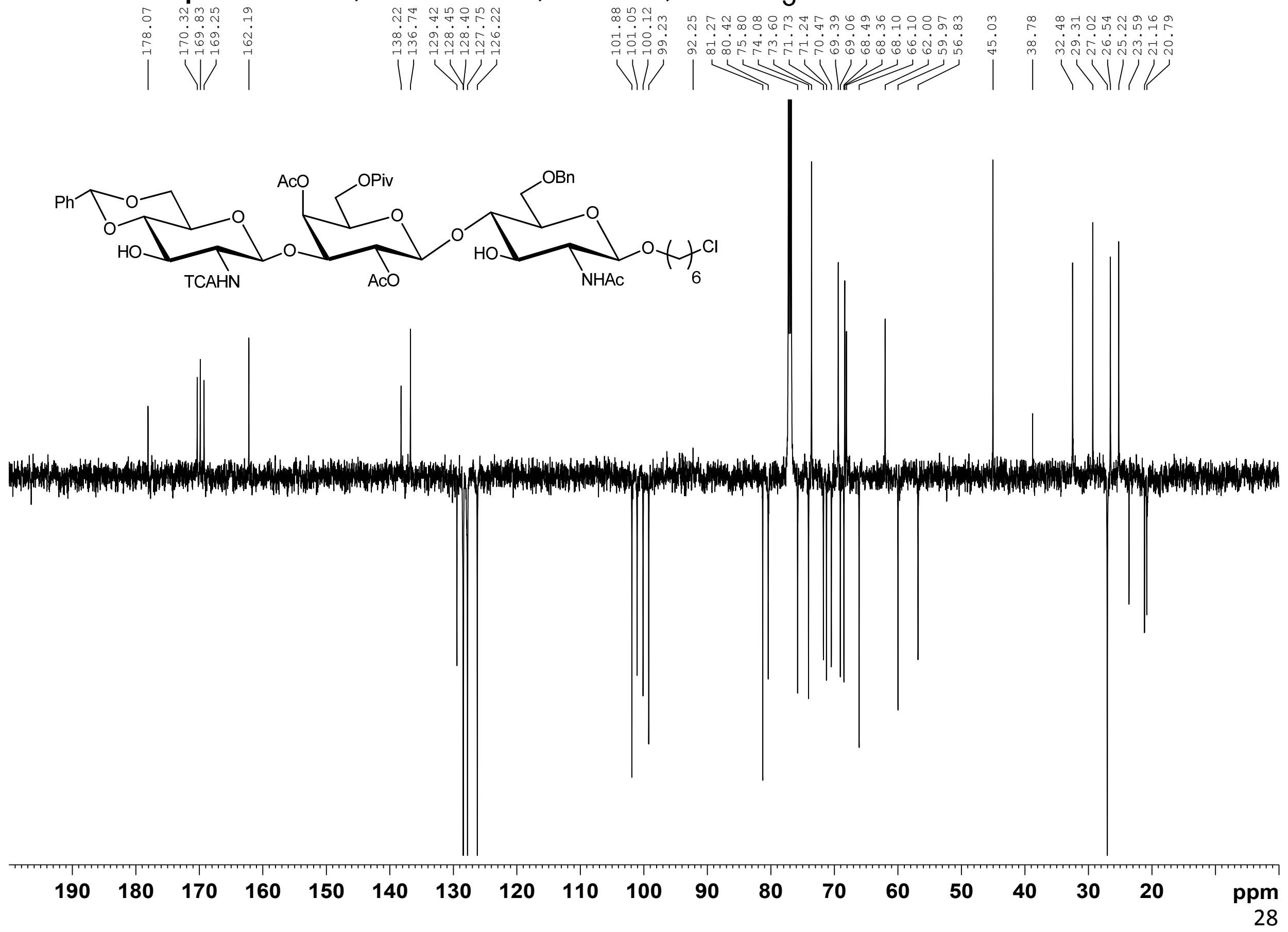


Compound 14, $125 \mathrm{MHz}, 296 \mathrm{~K}, \mathrm{CDCl}_{3}$

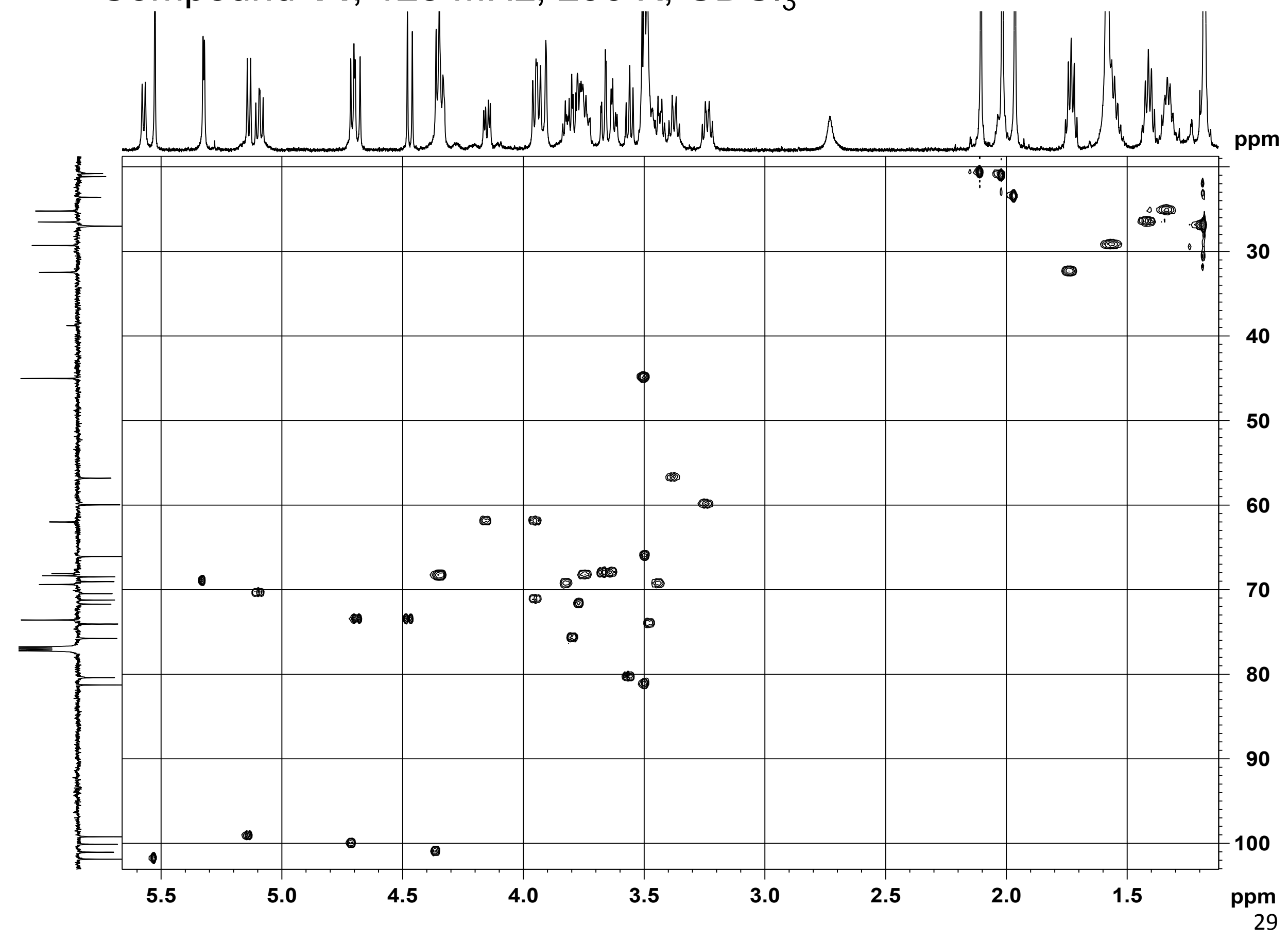




\section{Compound 15, $600 \mathrm{MHz}, 295 \mathrm{~K}, \mathrm{CDCl}_{3}$}

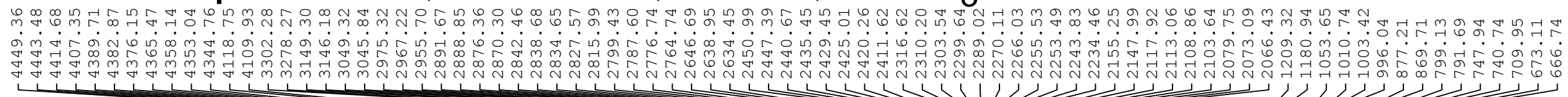

Tाmm

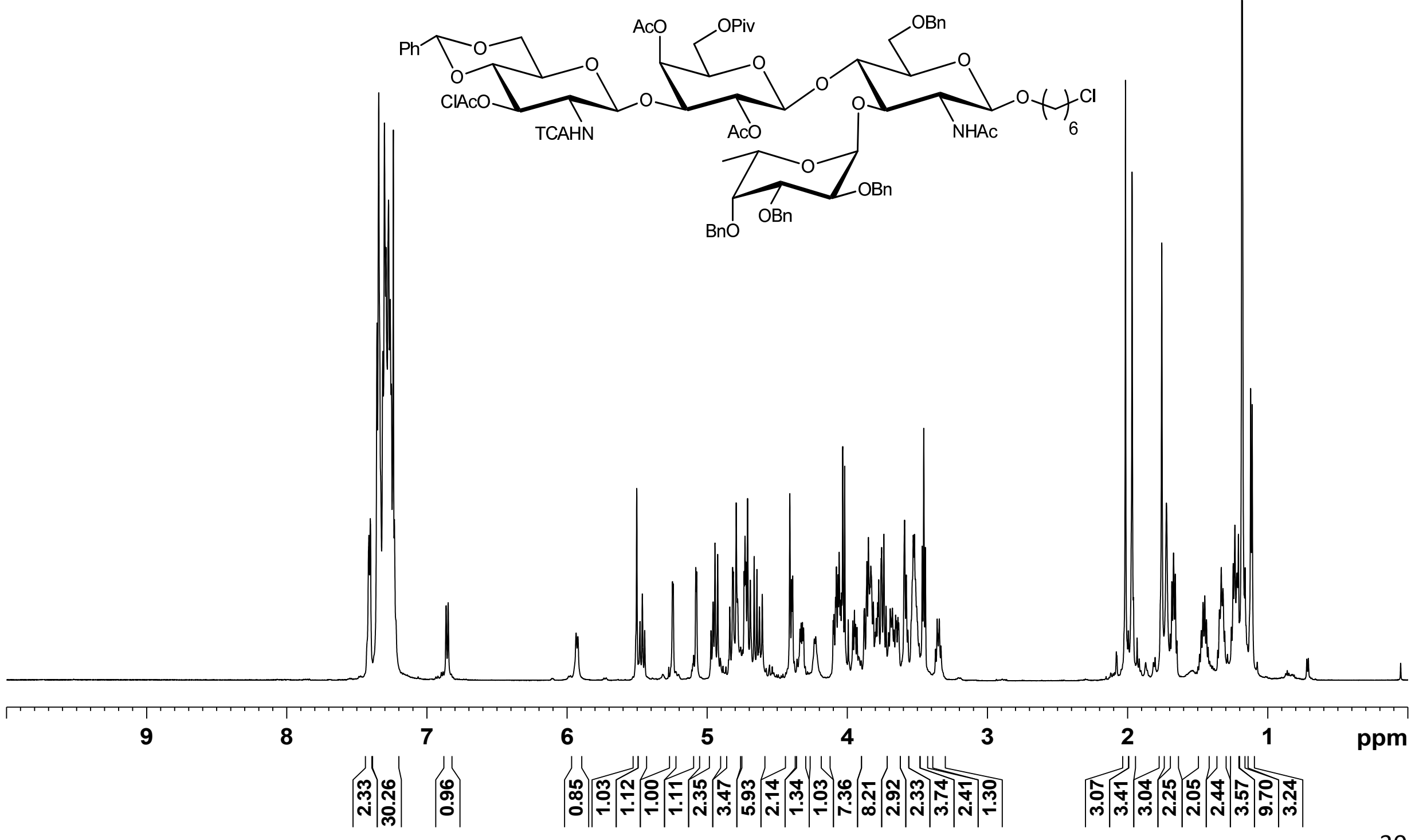


Compound 15, $600 \mathrm{MHz}, 295 \mathrm{~K}, \mathrm{CDCl}_{3}$

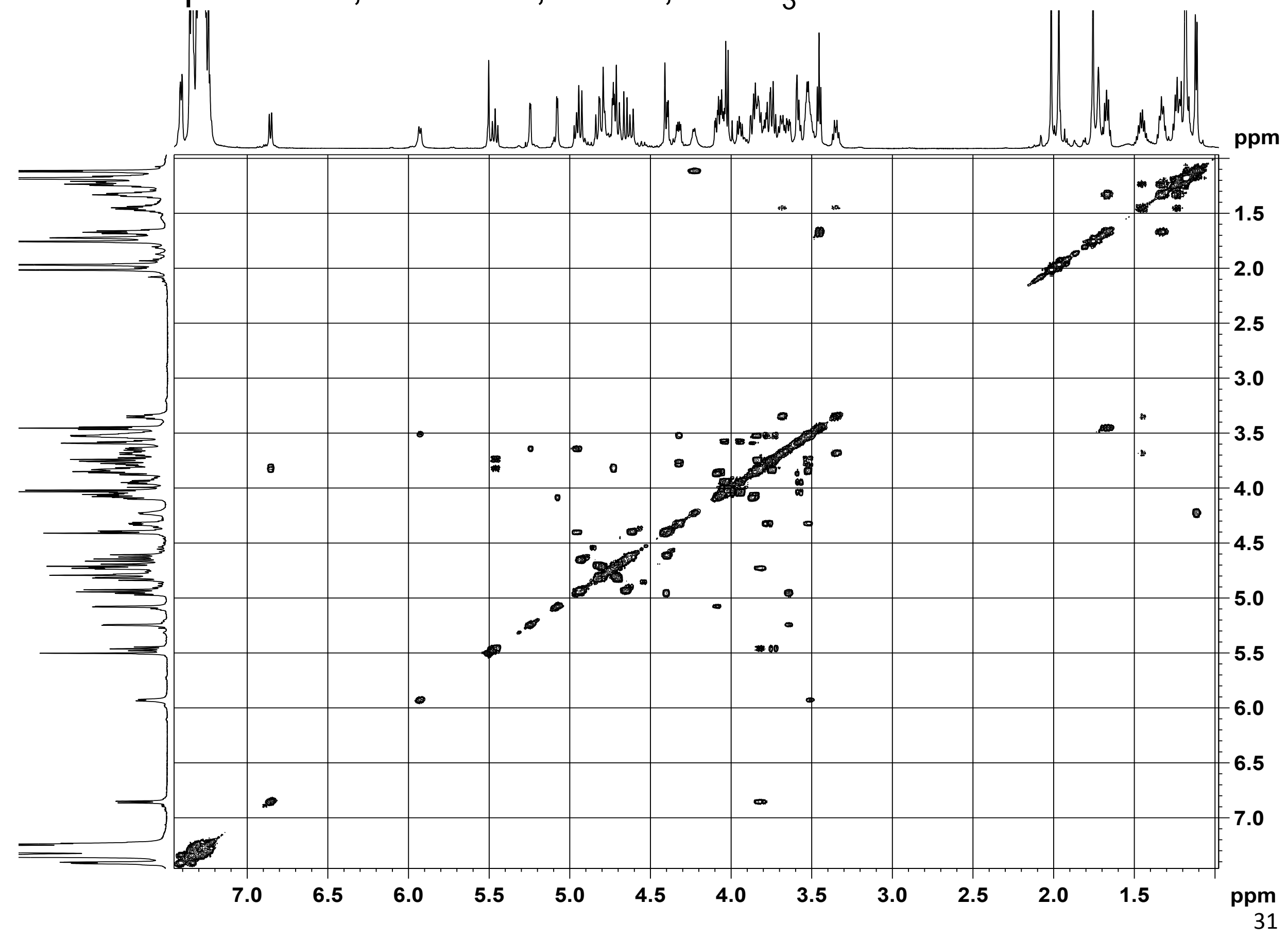


Compound 15, $125 \mathrm{MHz}, 295 \mathrm{~K}, \mathrm{CDCl}_{3}$

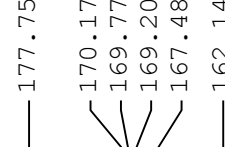

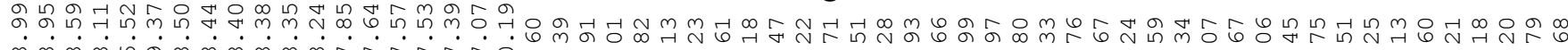
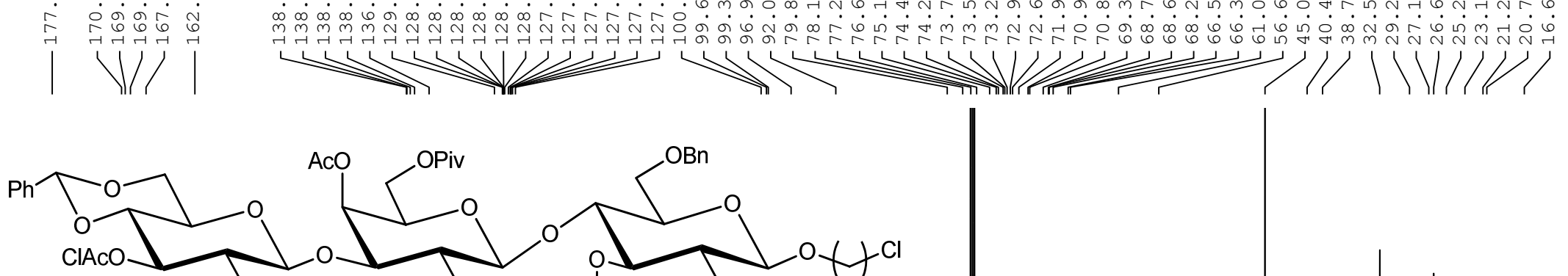

TCAHN

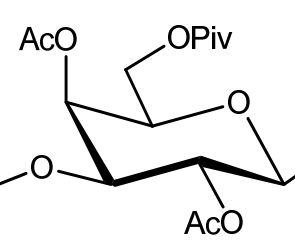

Bn
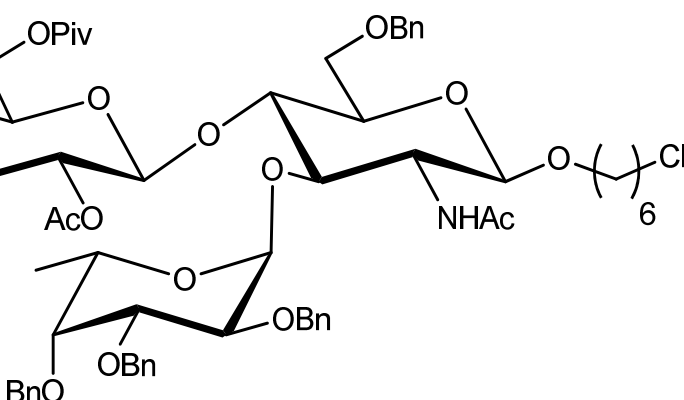

$\mathrm{BnO}$
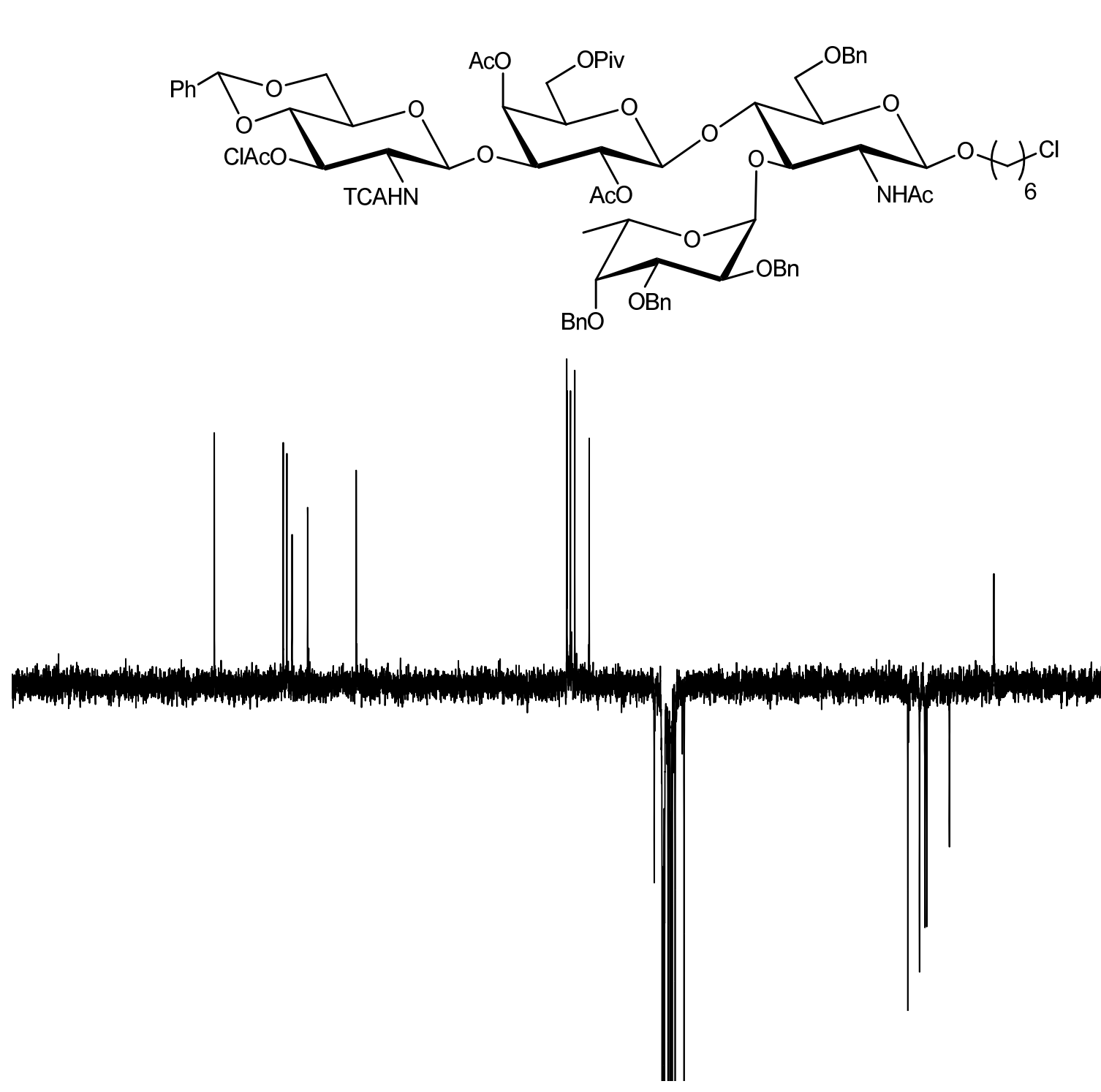
Compound 15, $125 \mathrm{MHz}, 295 \mathrm{~K}, \mathrm{CDCl}_{3}$

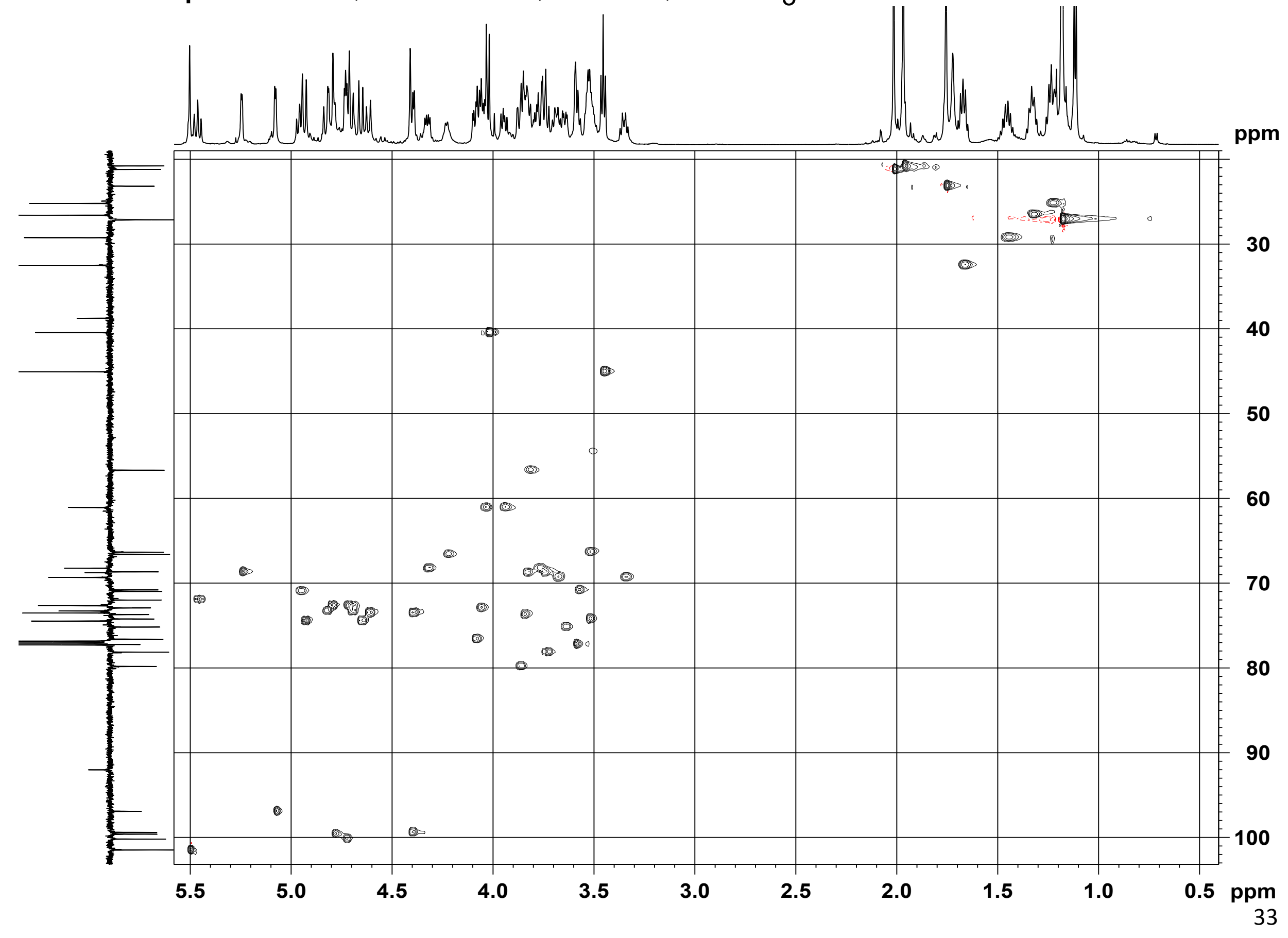




\section{Compound 16, $600 \mathrm{MHz}, 295 \mathrm{~K}, \mathrm{CDCl}_{3}$}

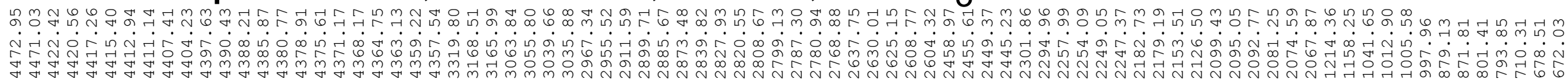

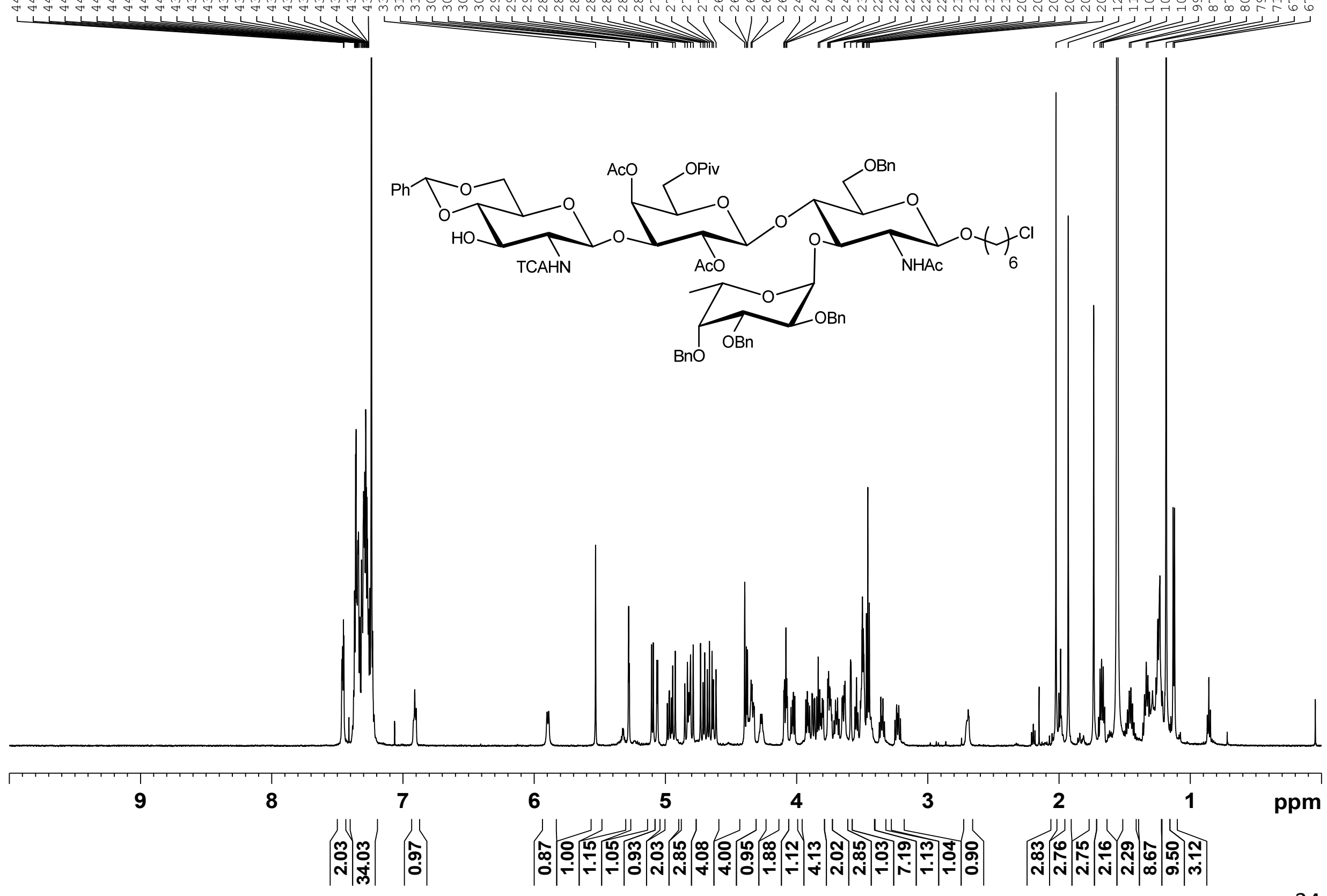


Compound 16, $600 \mathrm{MHz}, 295 \mathrm{~K}, \mathrm{CDCl}_{3}$

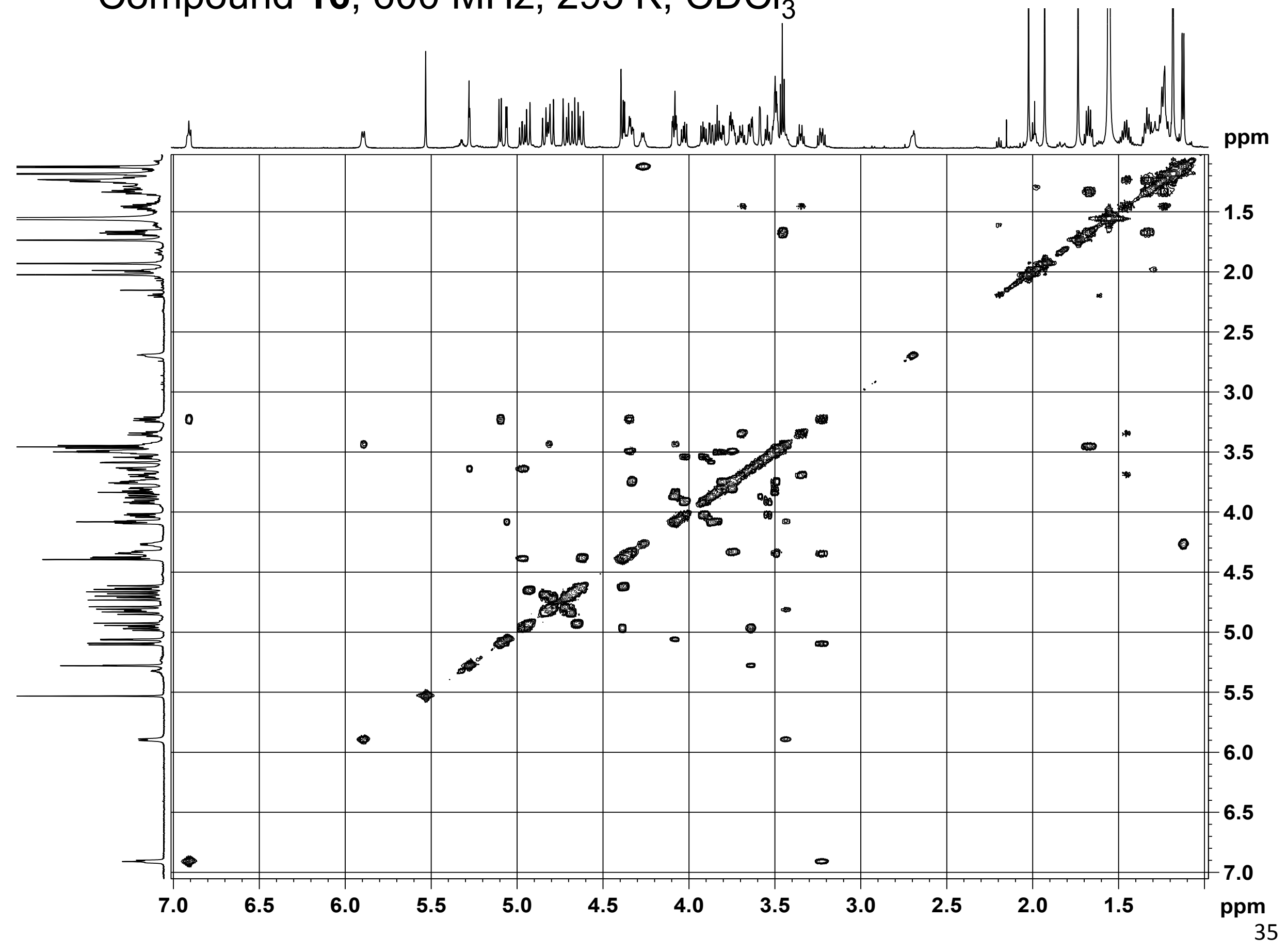


Compound 16, $125 \mathrm{MHz}, 295 \mathrm{~K}, \mathrm{CDCl}_{3}$

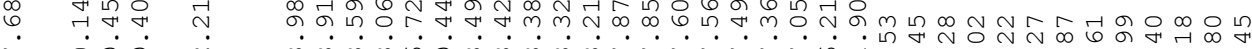
占

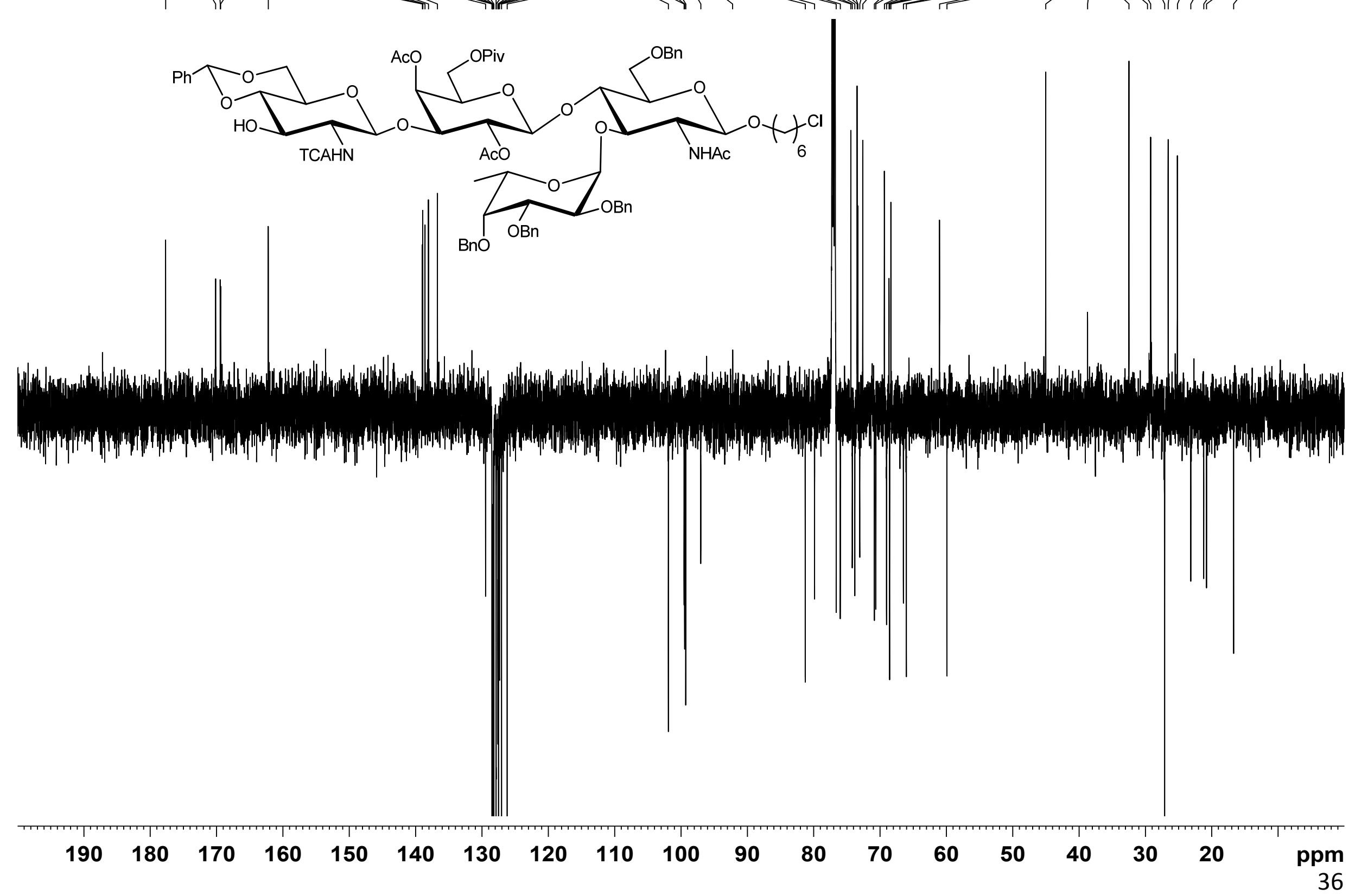


Compound 16, $125 \mathrm{MHz}, 295 \mathrm{~K}, \mathrm{CDCl}_{3}$

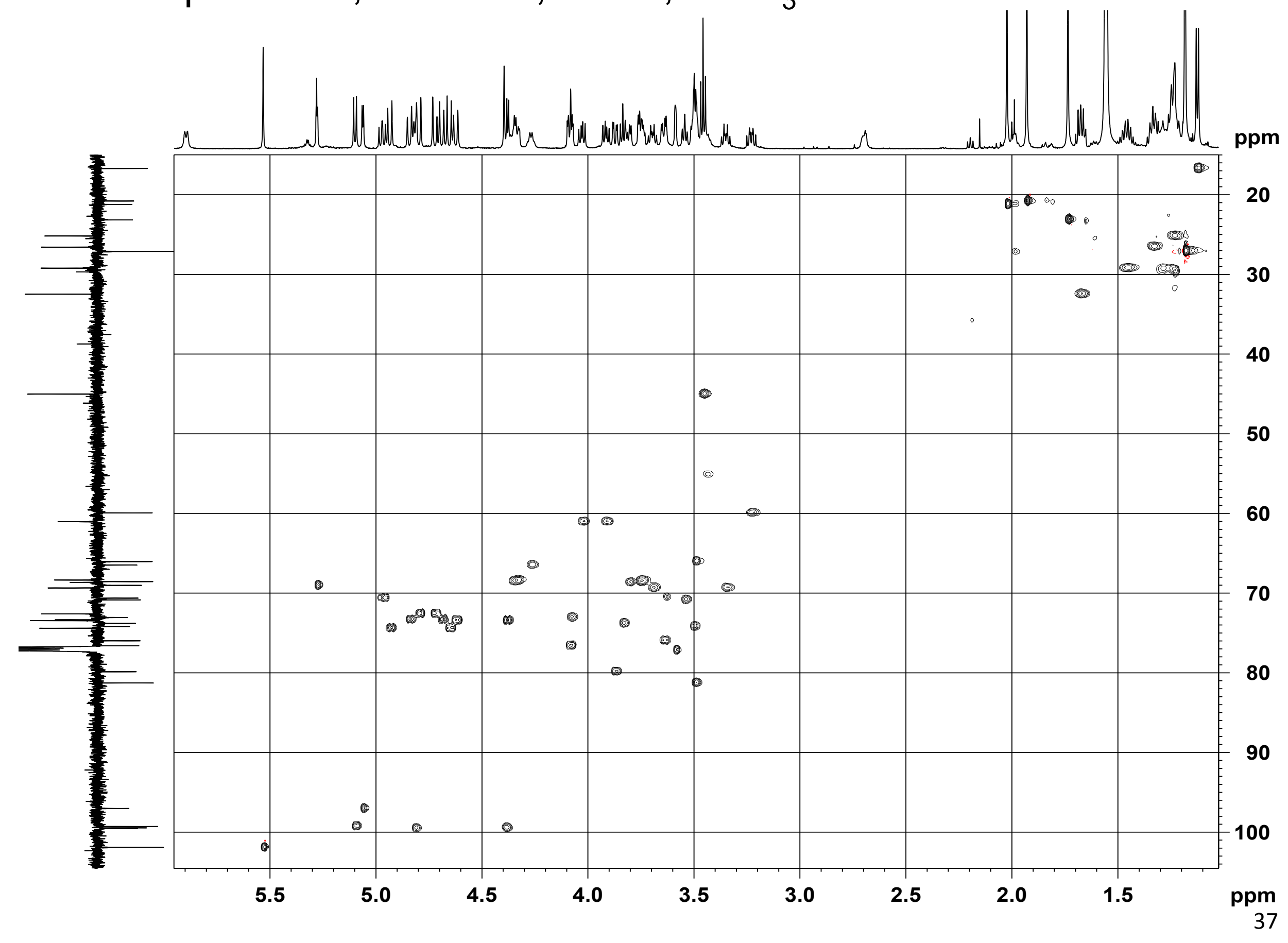




\section{Compound 17, $600 \mathrm{MHz}, 295 \mathrm{~K}, \mathrm{CDCl}_{3}$}

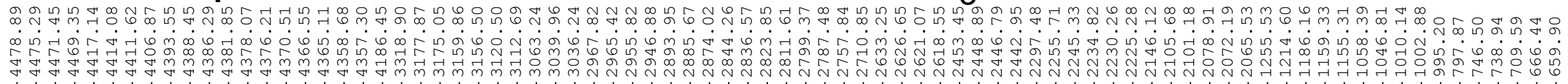

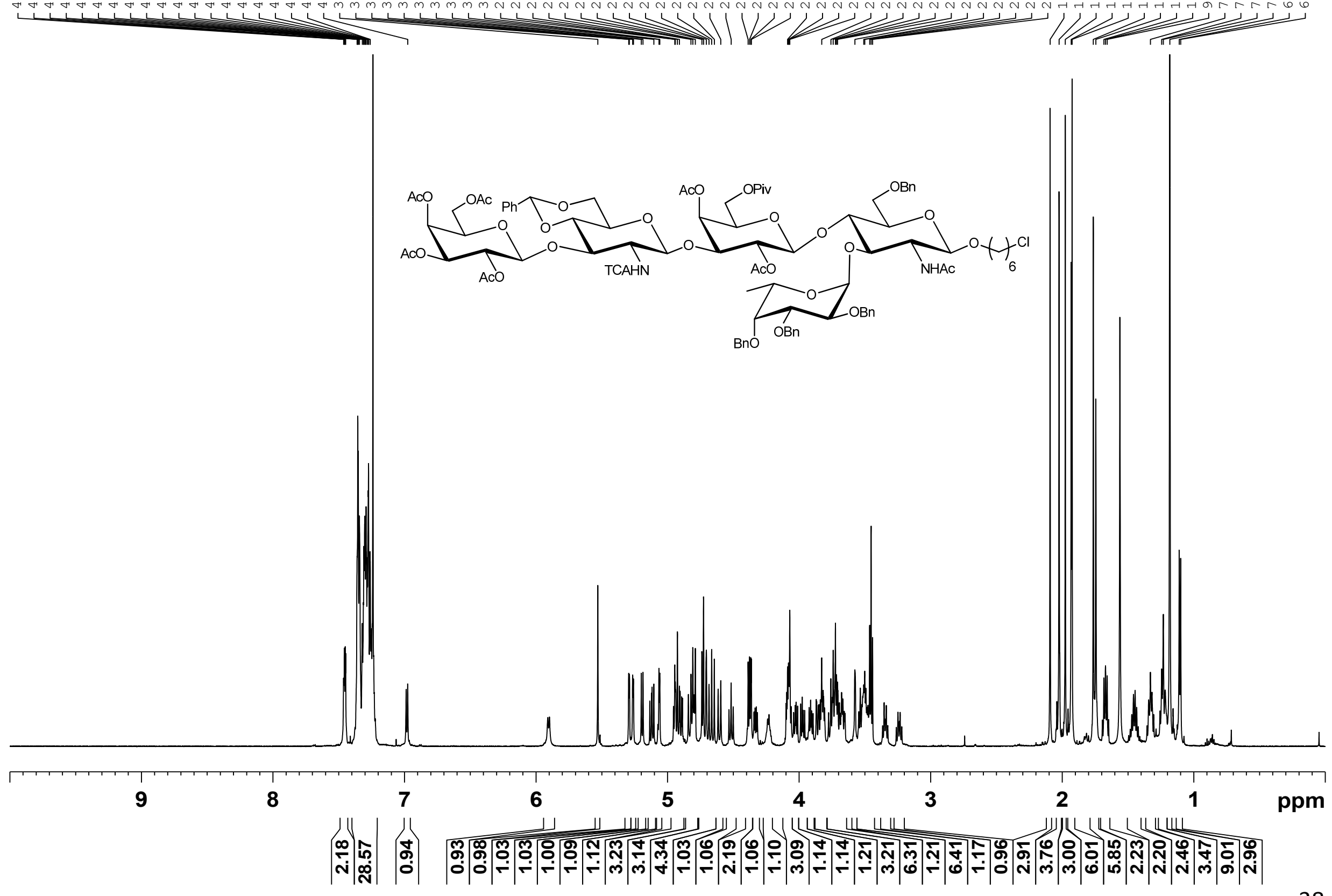


Compound 17, $600 \mathrm{MHz}, 295 \mathrm{~K}, \mathrm{CDCl}_{3}$

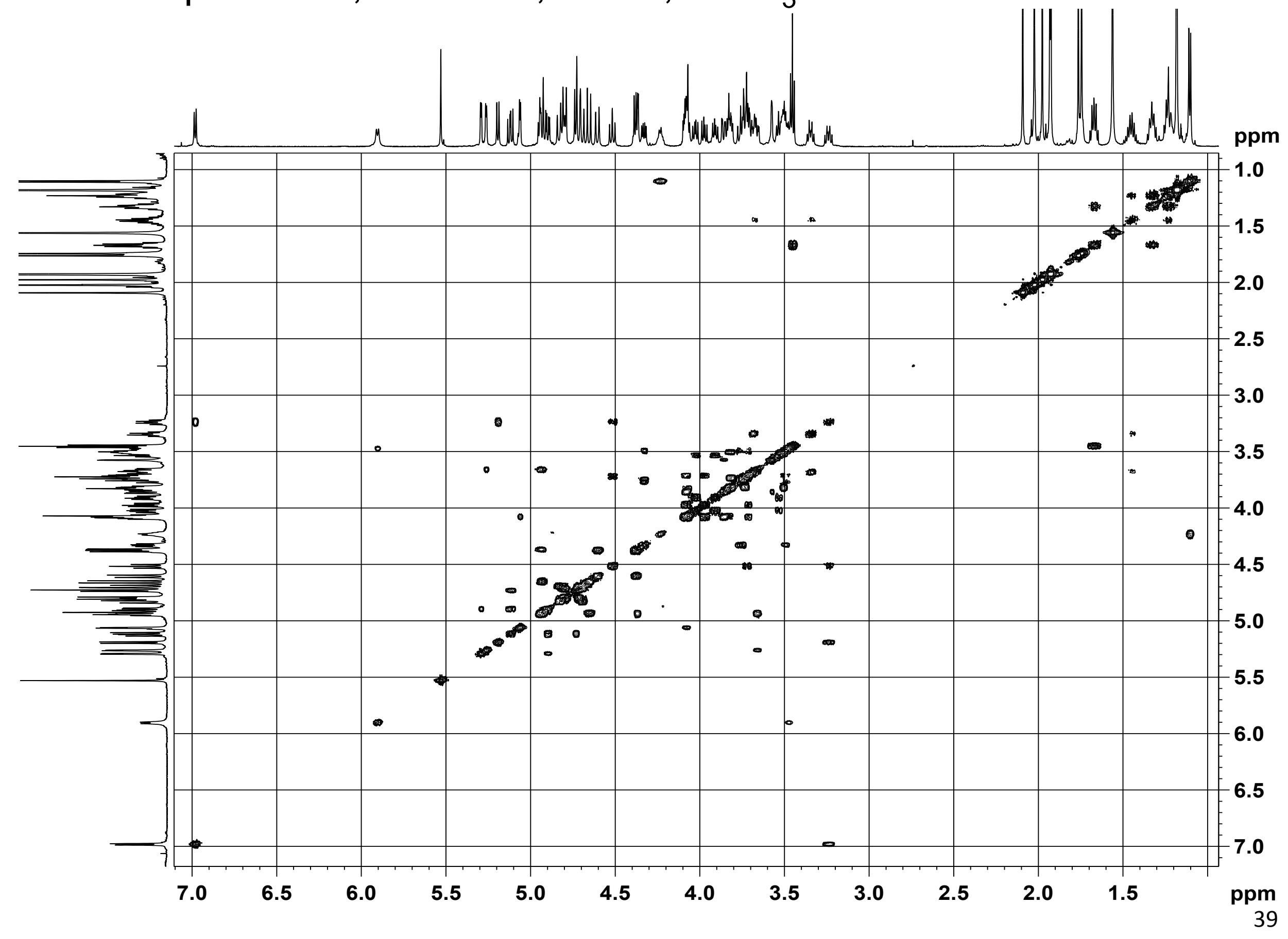




\section{Compound 17, $125 \mathrm{MHz}, 295 \mathrm{~K}, \mathrm{CDCl}_{3}$}

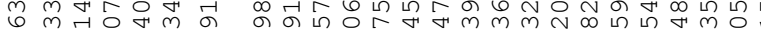

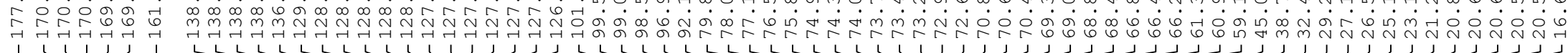

|

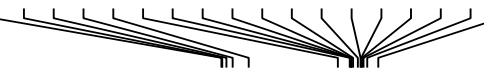

$\mathrm{AcO}$

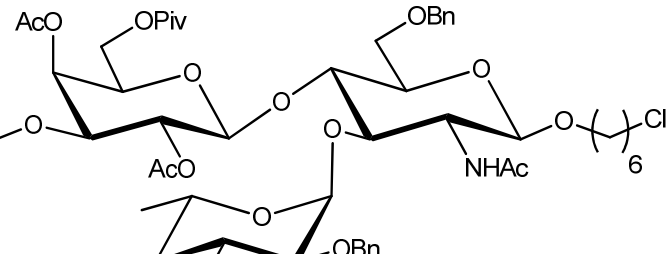

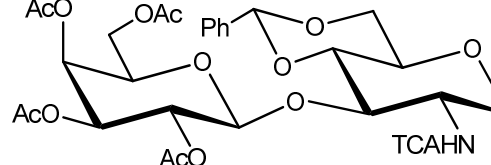

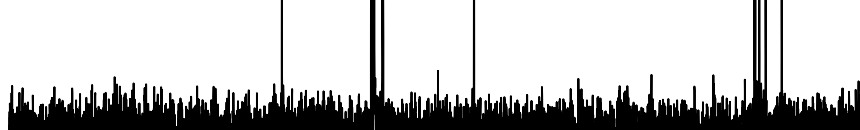
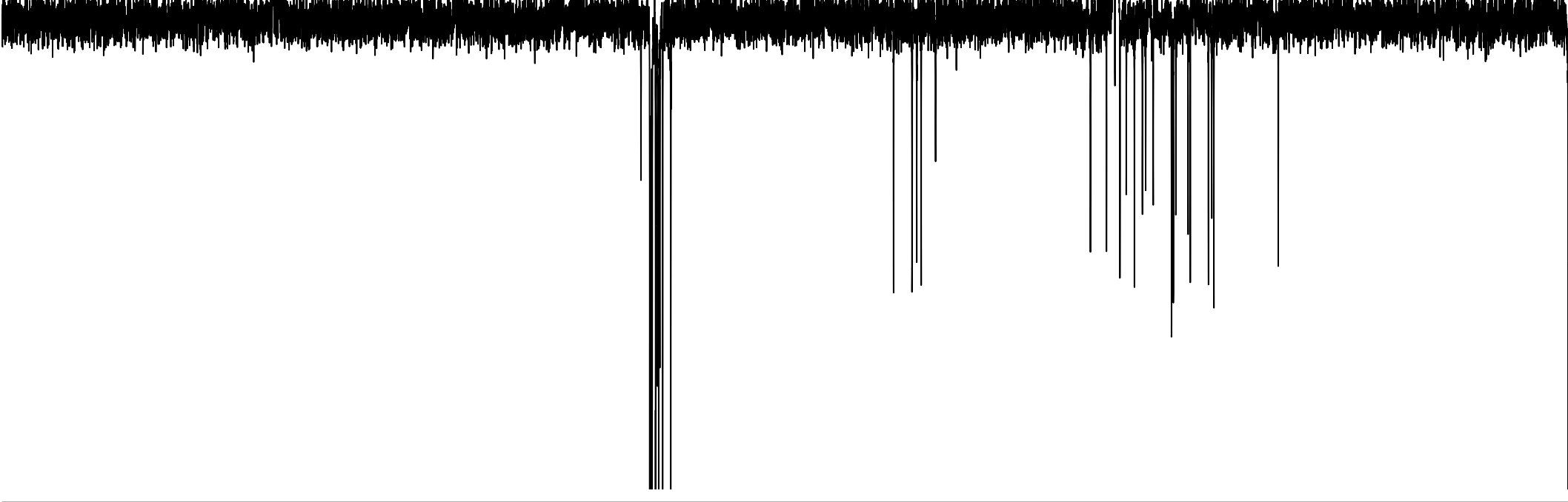
Compound 17, $125 \mathrm{MHz}, 295 \mathrm{~K}, \mathrm{CDCl}_{3}$

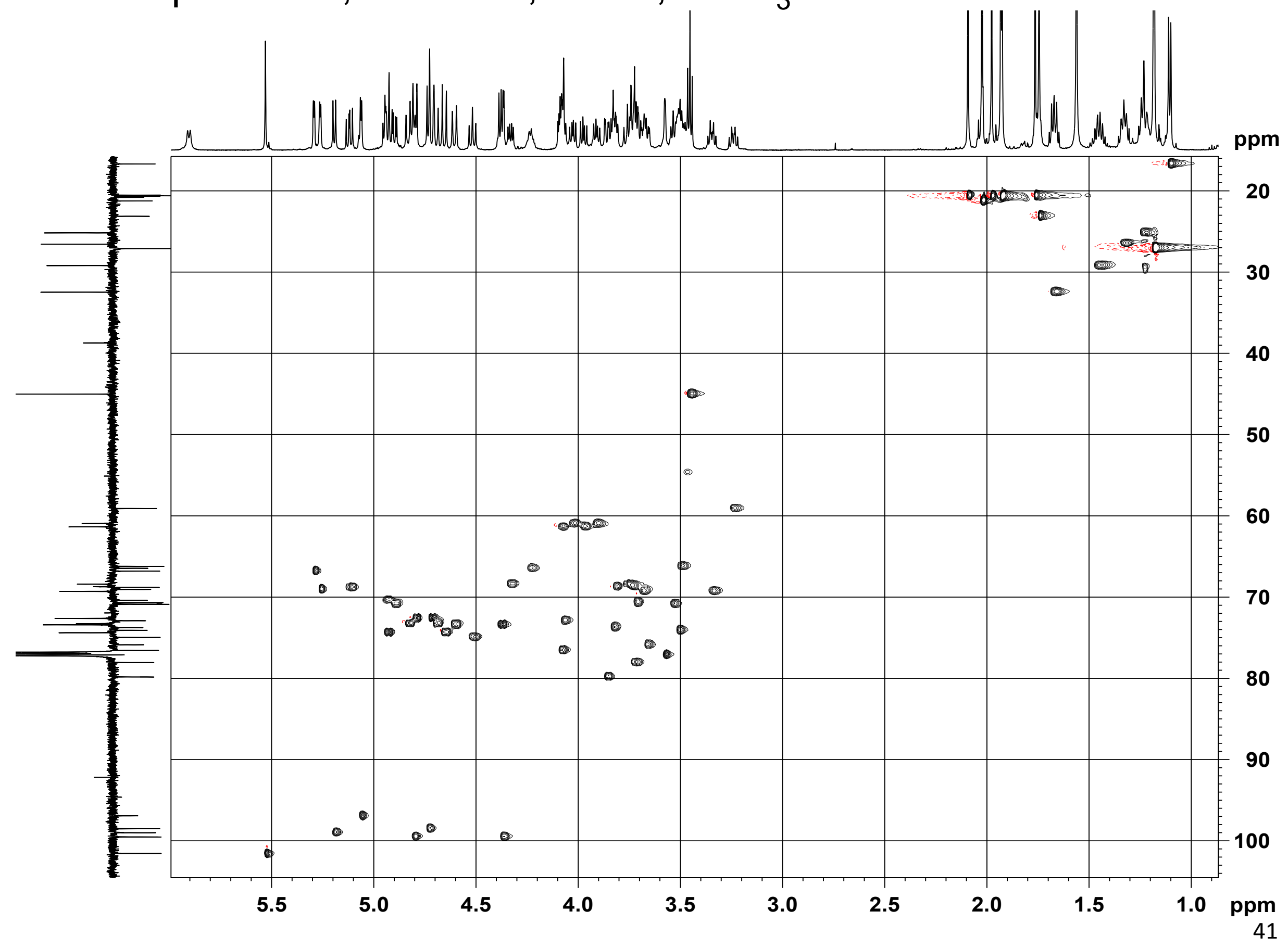




\section{Compound 18, $600 \mathrm{MHz}, 295 \mathrm{~K}, \mathrm{CDCl}_{3}$}

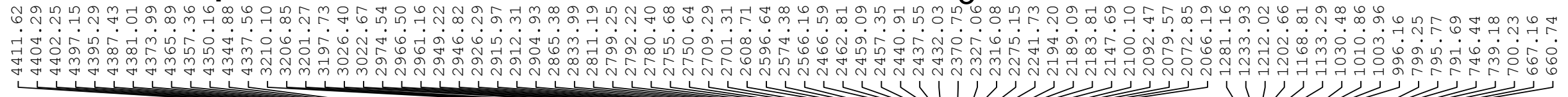

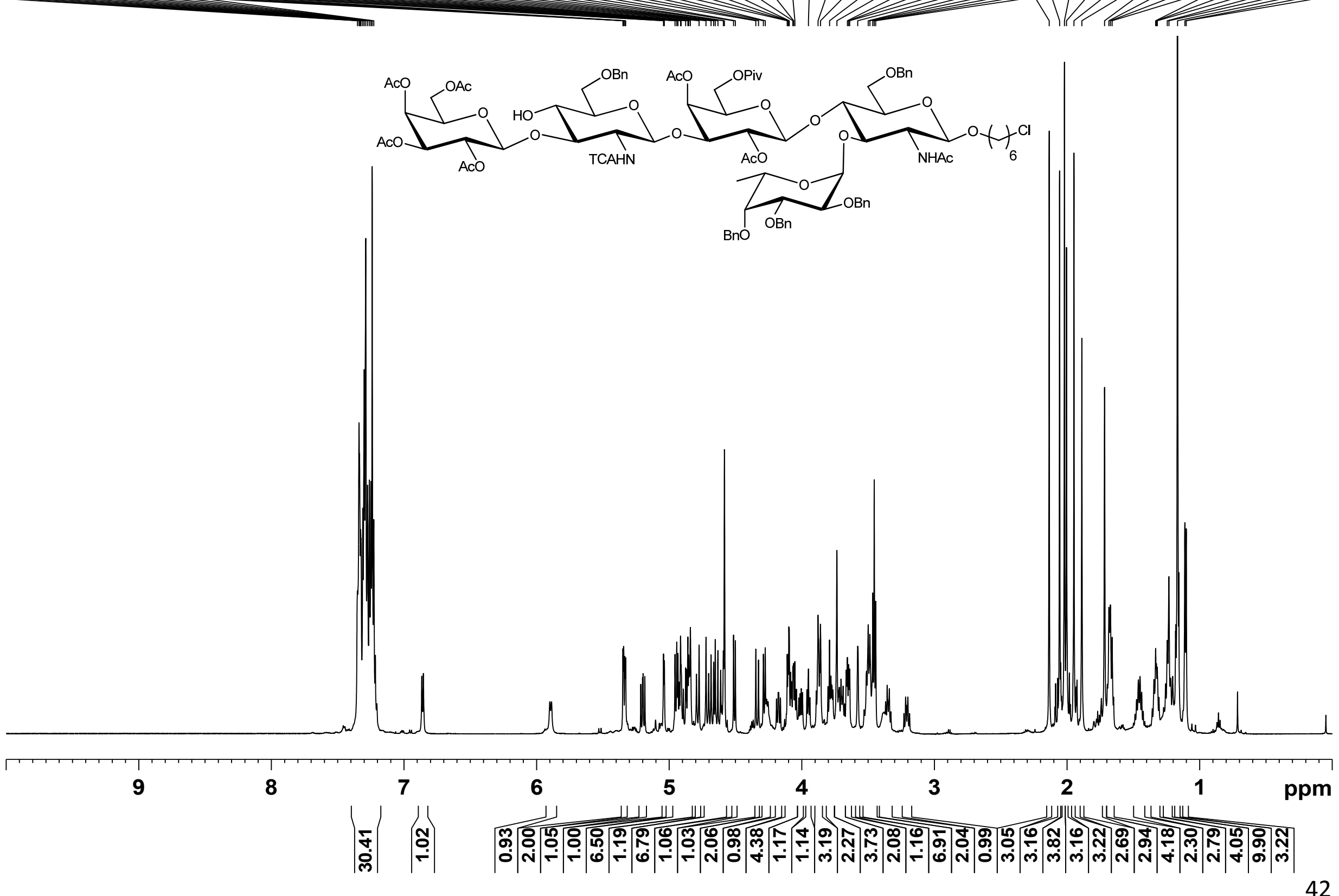


Compound 18, $600 \mathrm{MHz}, 295 \mathrm{~K}, \mathrm{CDCl}_{3}$

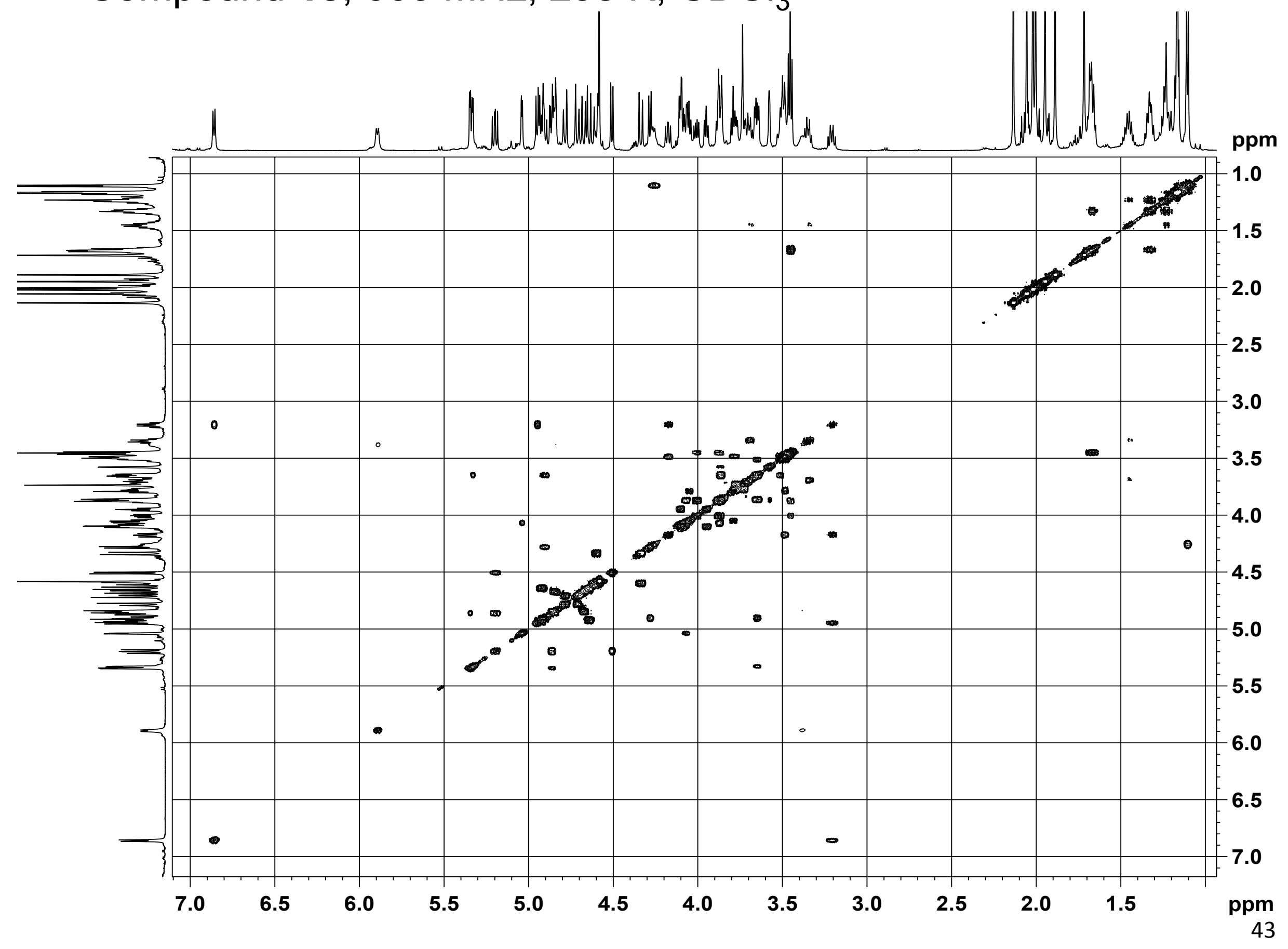


Compound 18, $125 \mathrm{MHz}, 295 \mathrm{~K}, \mathrm{CDCl}_{3}$

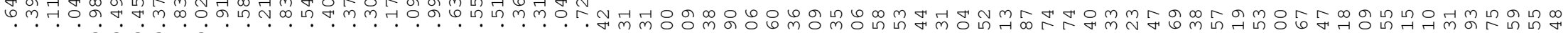

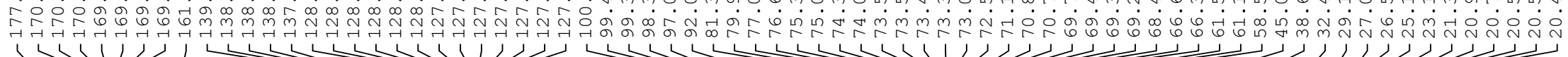
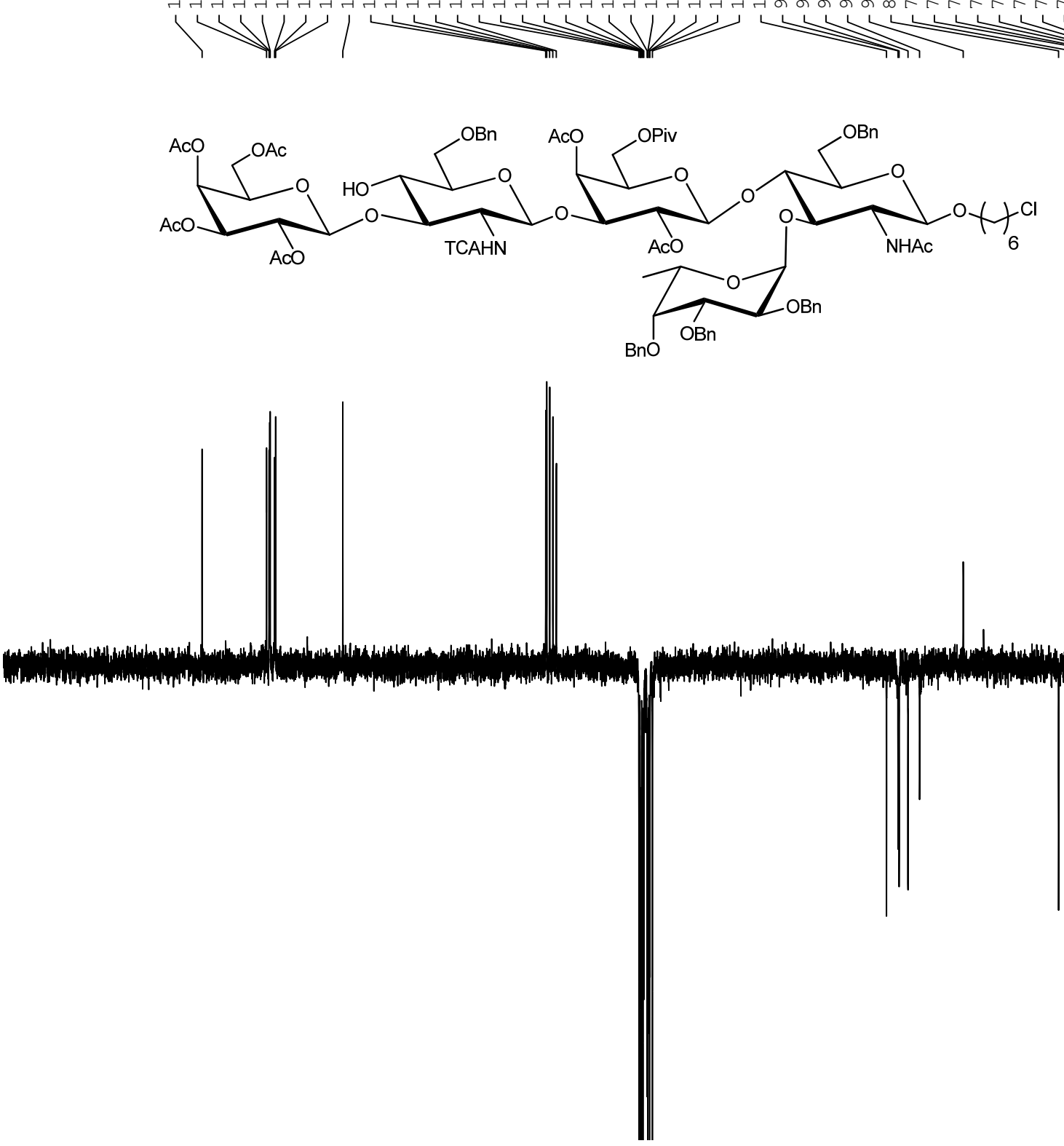
Compound 18, $125 \mathrm{MHz}, 295 \mathrm{~K}, \mathrm{CDCl}_{3}$

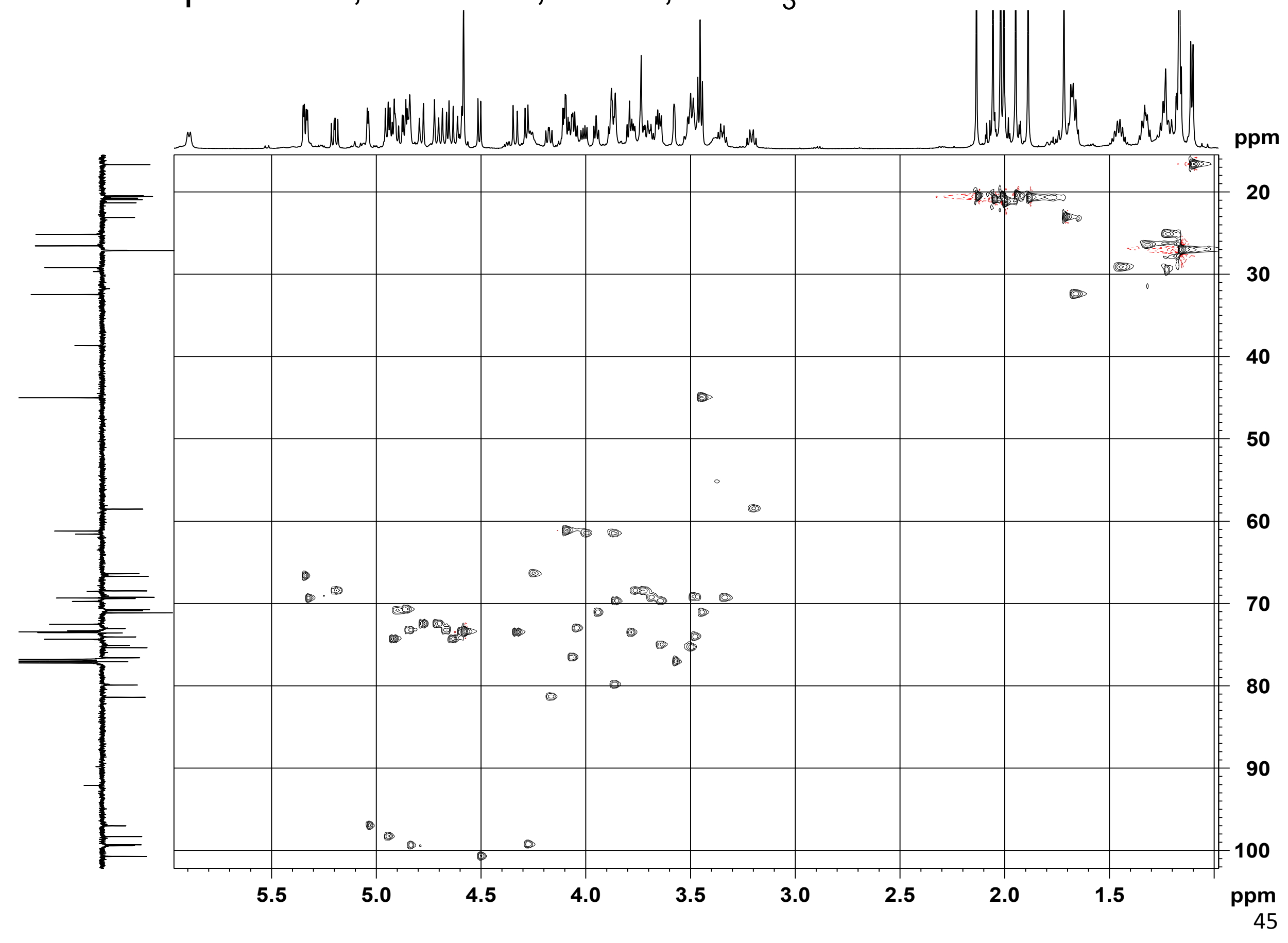




\section{Compound 19, $600 \mathrm{MHz}, 295 \mathrm{~K}, \mathrm{CDCl}_{3}$}

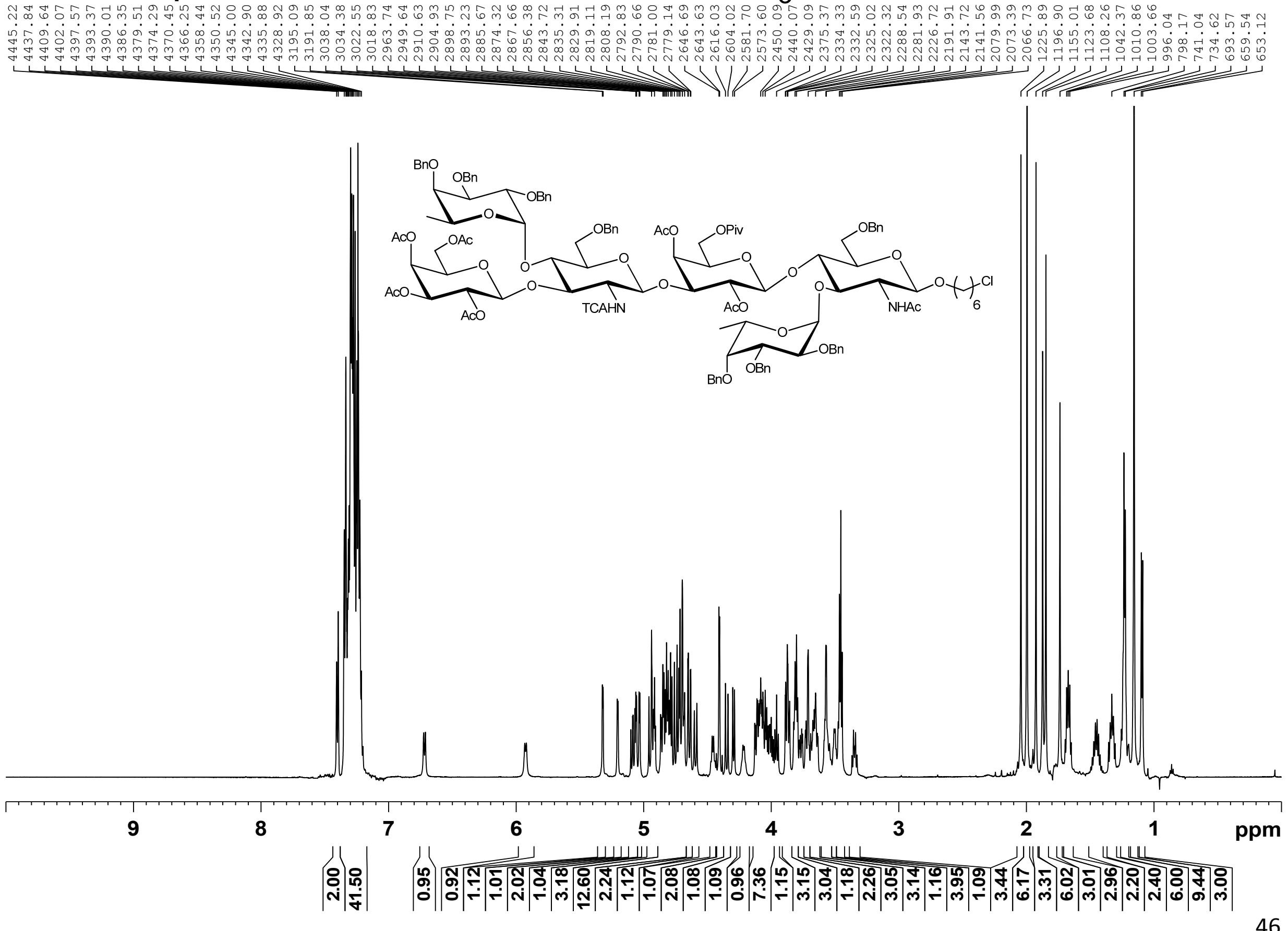


Compound 19, $600 \mathrm{MHz}, 295 \mathrm{~K}, \mathrm{CDCl}_{3}$

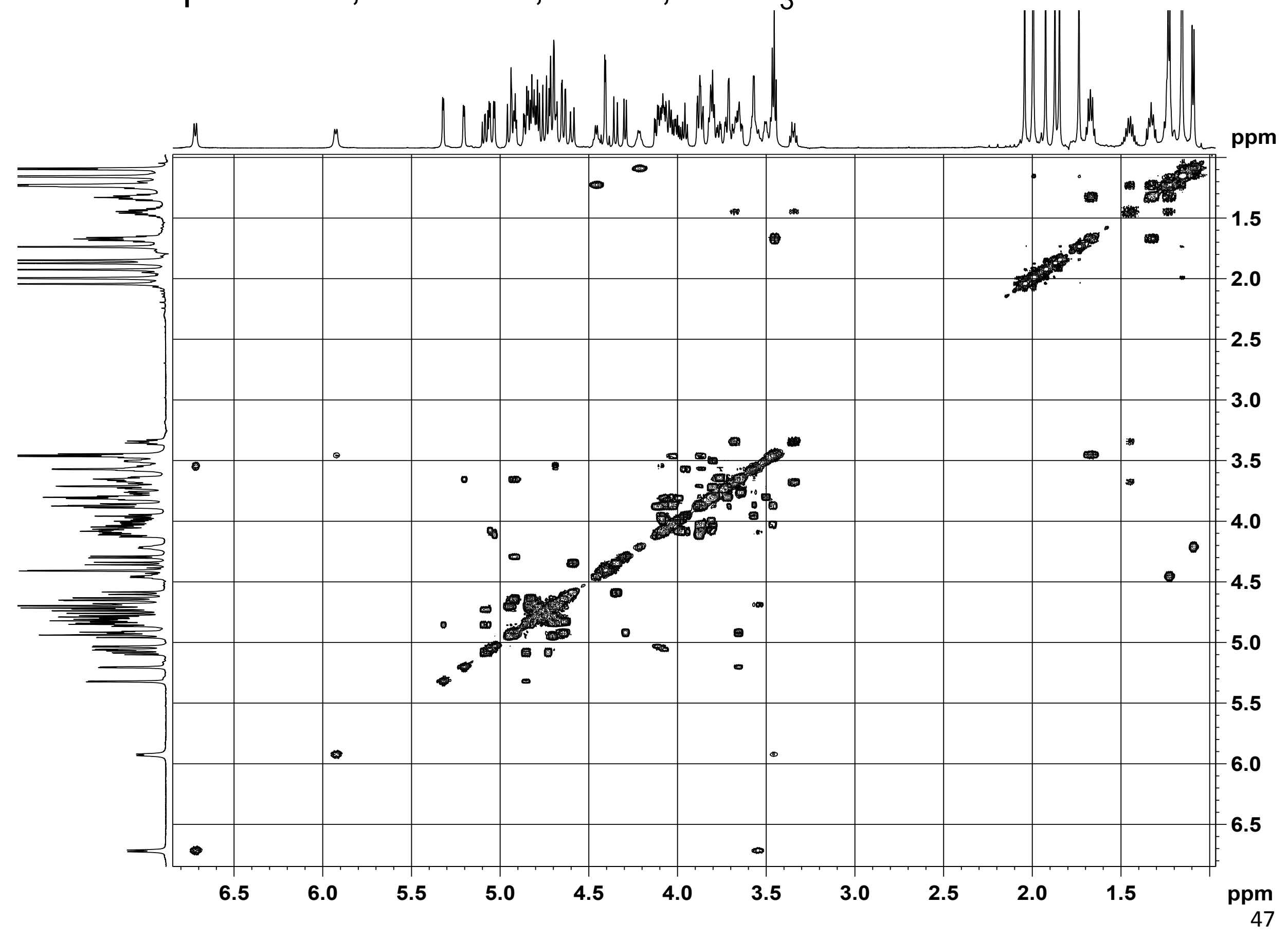




\section{Compound 19, $125 \mathrm{MHz}, 295 \mathrm{~K}, \mathrm{CDCl}_{3}$}

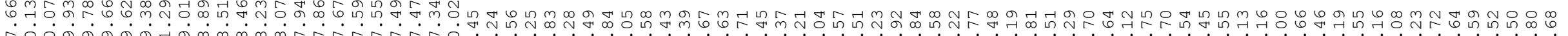

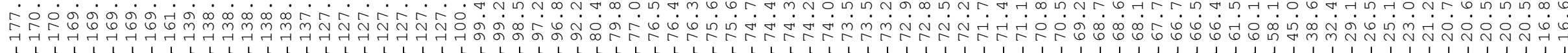

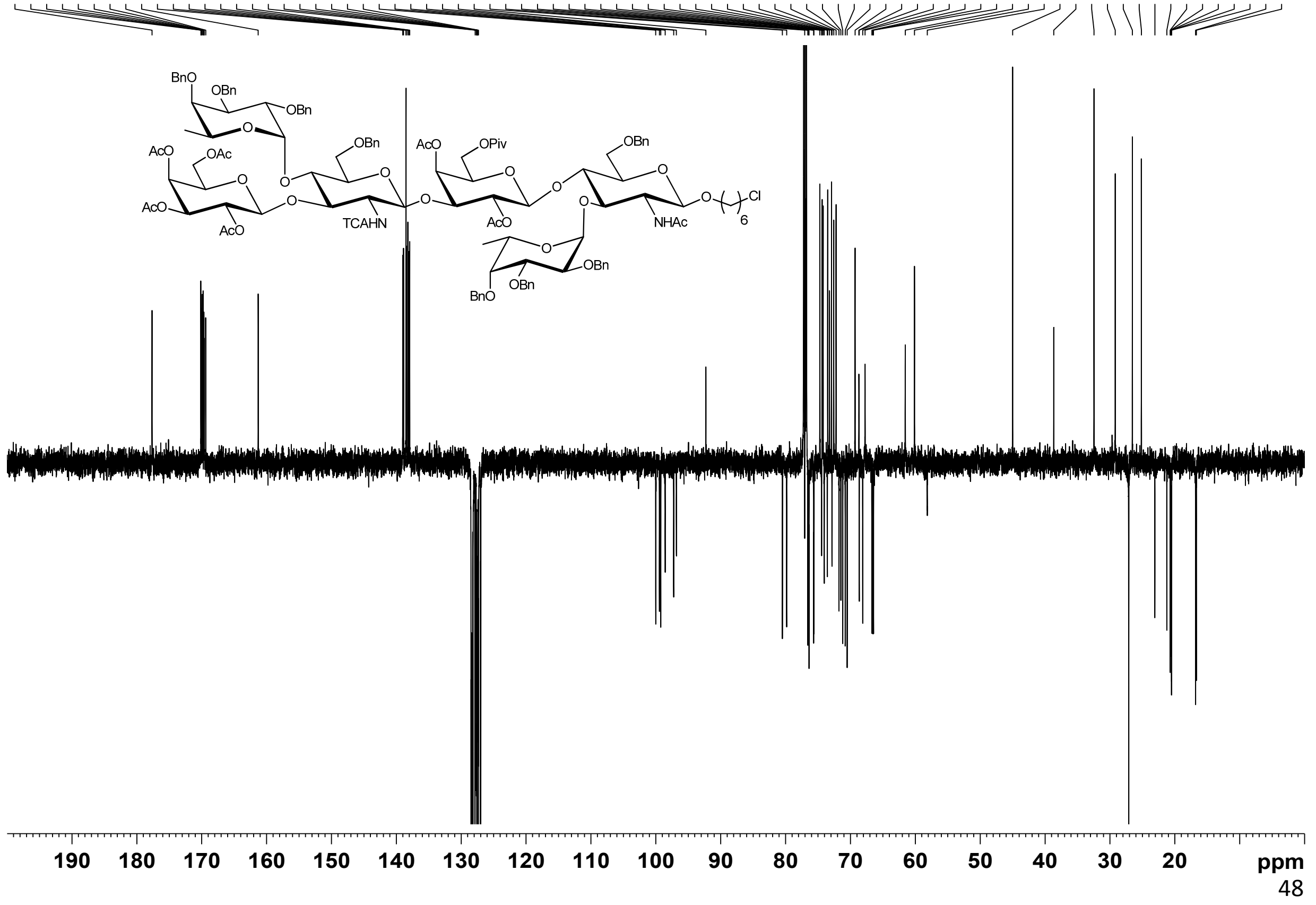


Compound 19, $125 \mathrm{MHz}, 295 \mathrm{~K}, \mathrm{CDCl}_{3}$

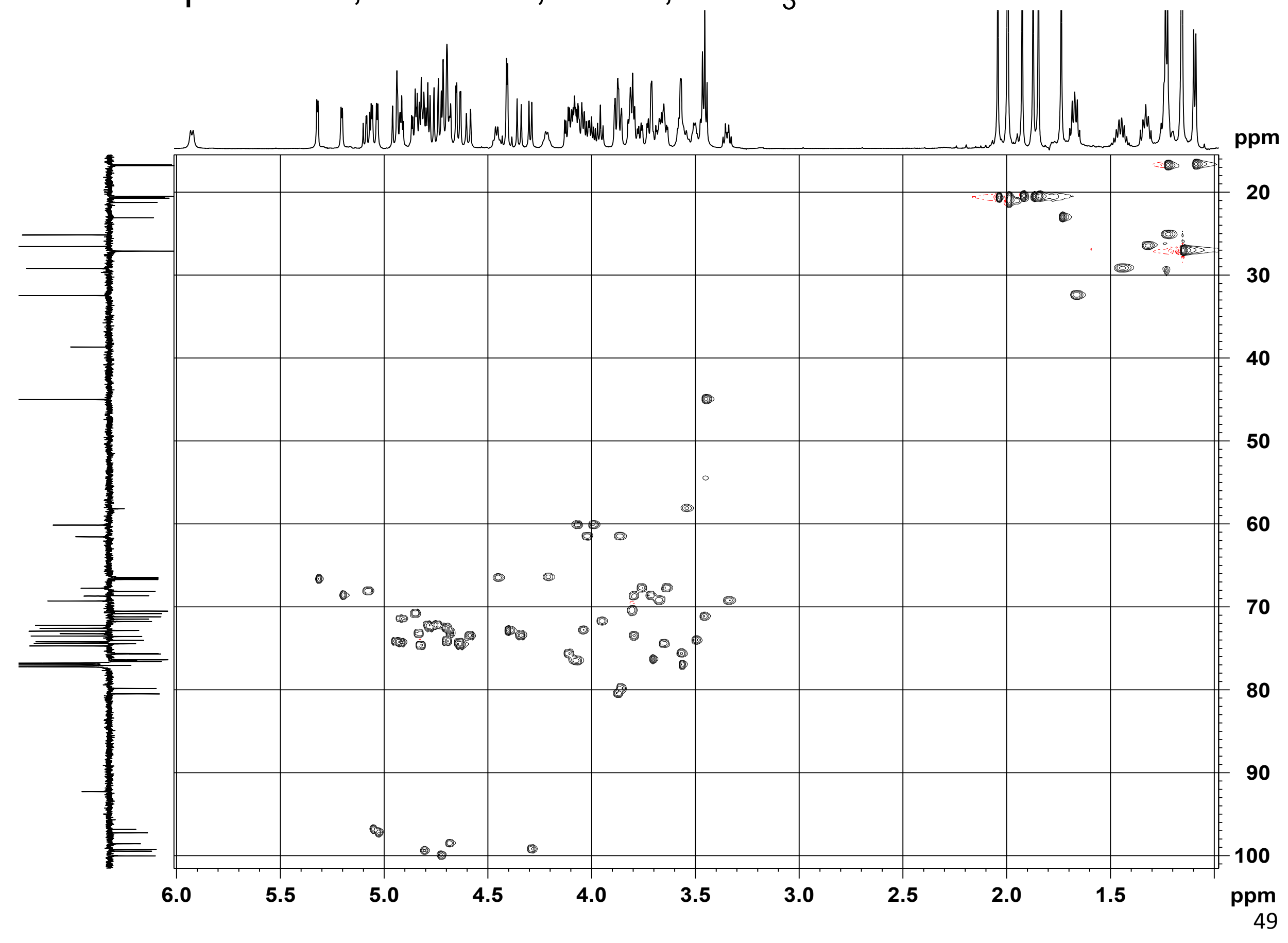




\section{Compound 20, $600 \mathrm{MHz}, 295 \mathrm{~K}, \mathrm{CDCl}_{3}$}

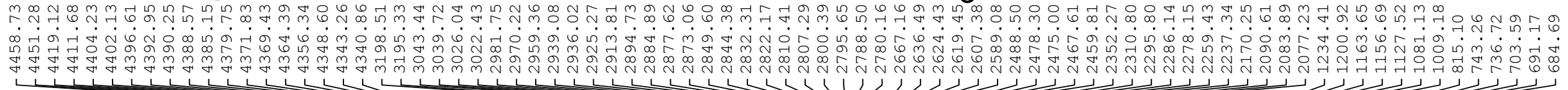

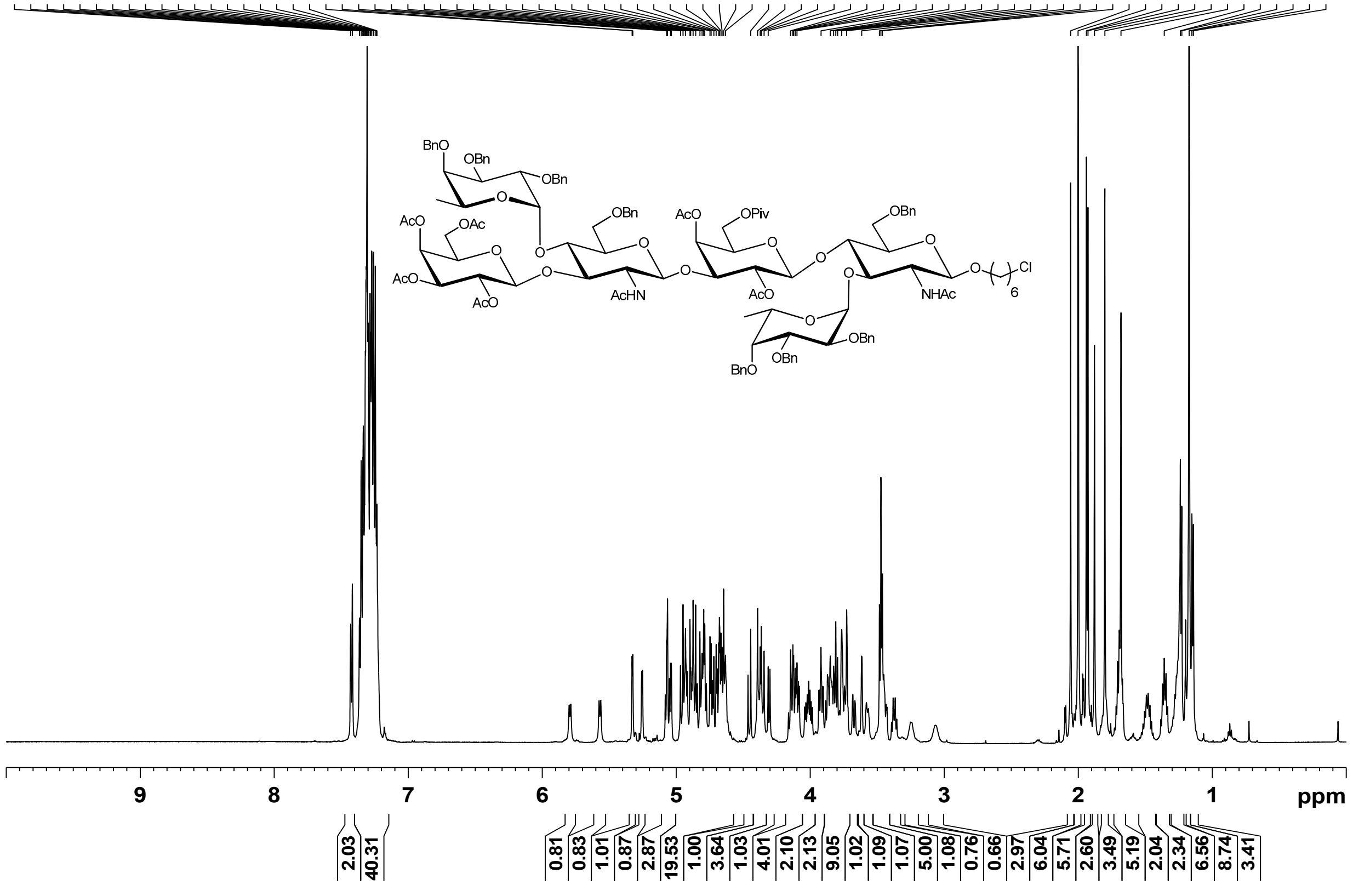


Compound 20, $600 \mathrm{MHz}, 295 \mathrm{~K}, \mathrm{CDCl}_{3}$

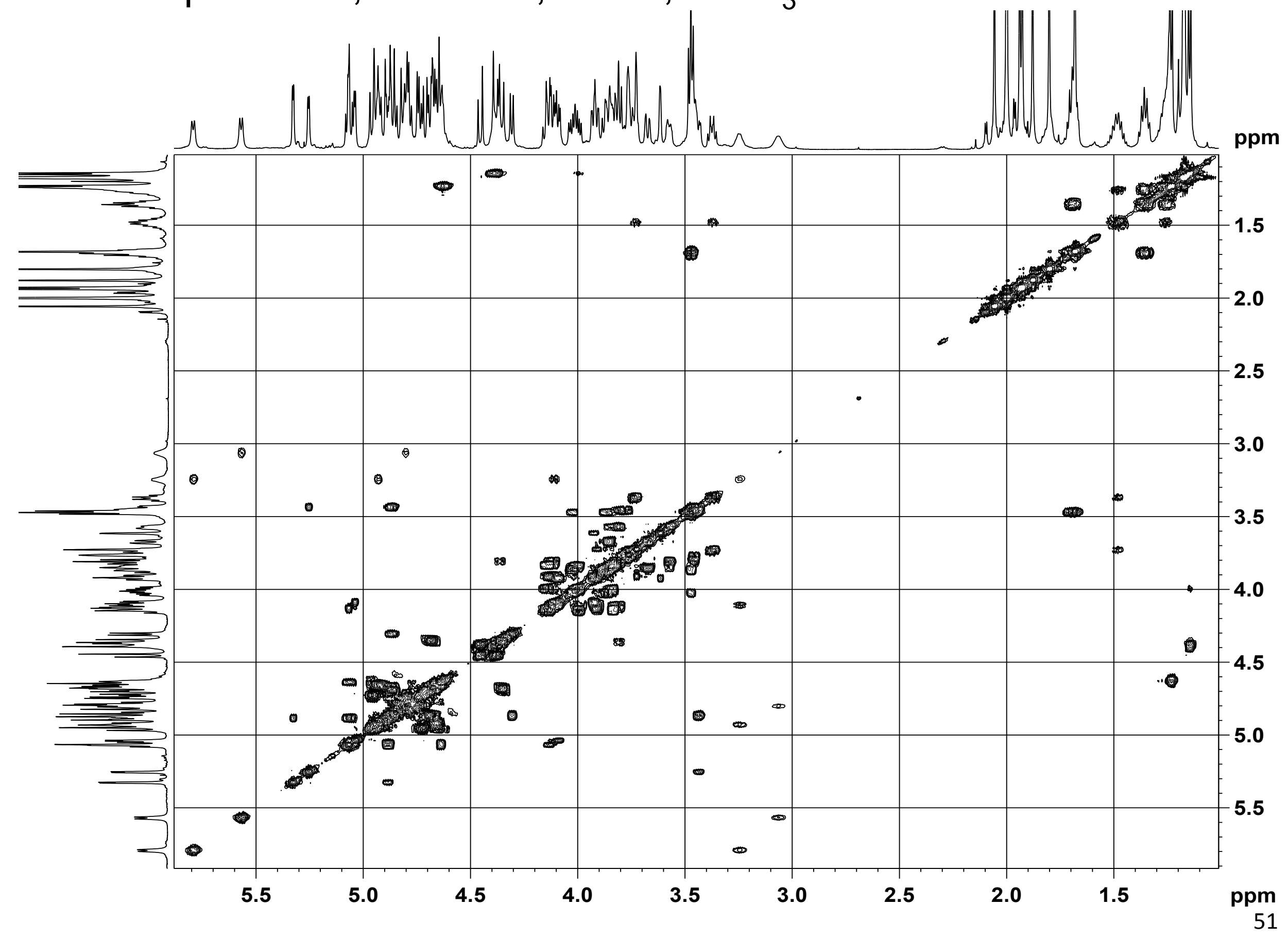


Compound 20, $125 \mathrm{MHz}, 295 \mathrm{~K}, \mathrm{CDCl}_{3}$

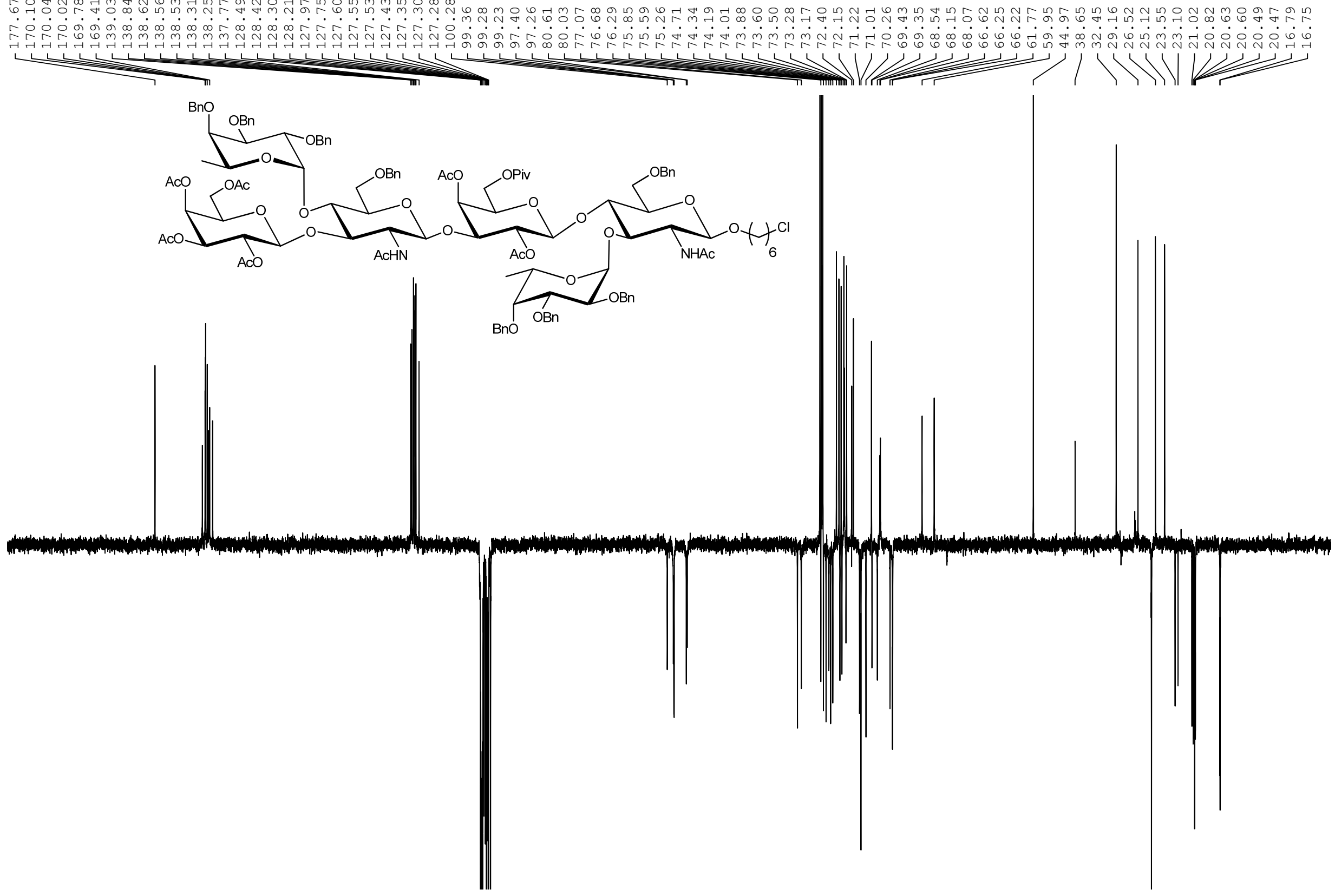

$\begin{array}{lllllll}190 & 180 & 170 & 160 & 150 & 140 & 130\end{array}$

120

110100

90

80

70

60

50

40

$30 \quad 20$ 
Compound 20, $125 \mathrm{MHz}, 295 \mathrm{~K}, \mathrm{CDCl}_{3}$

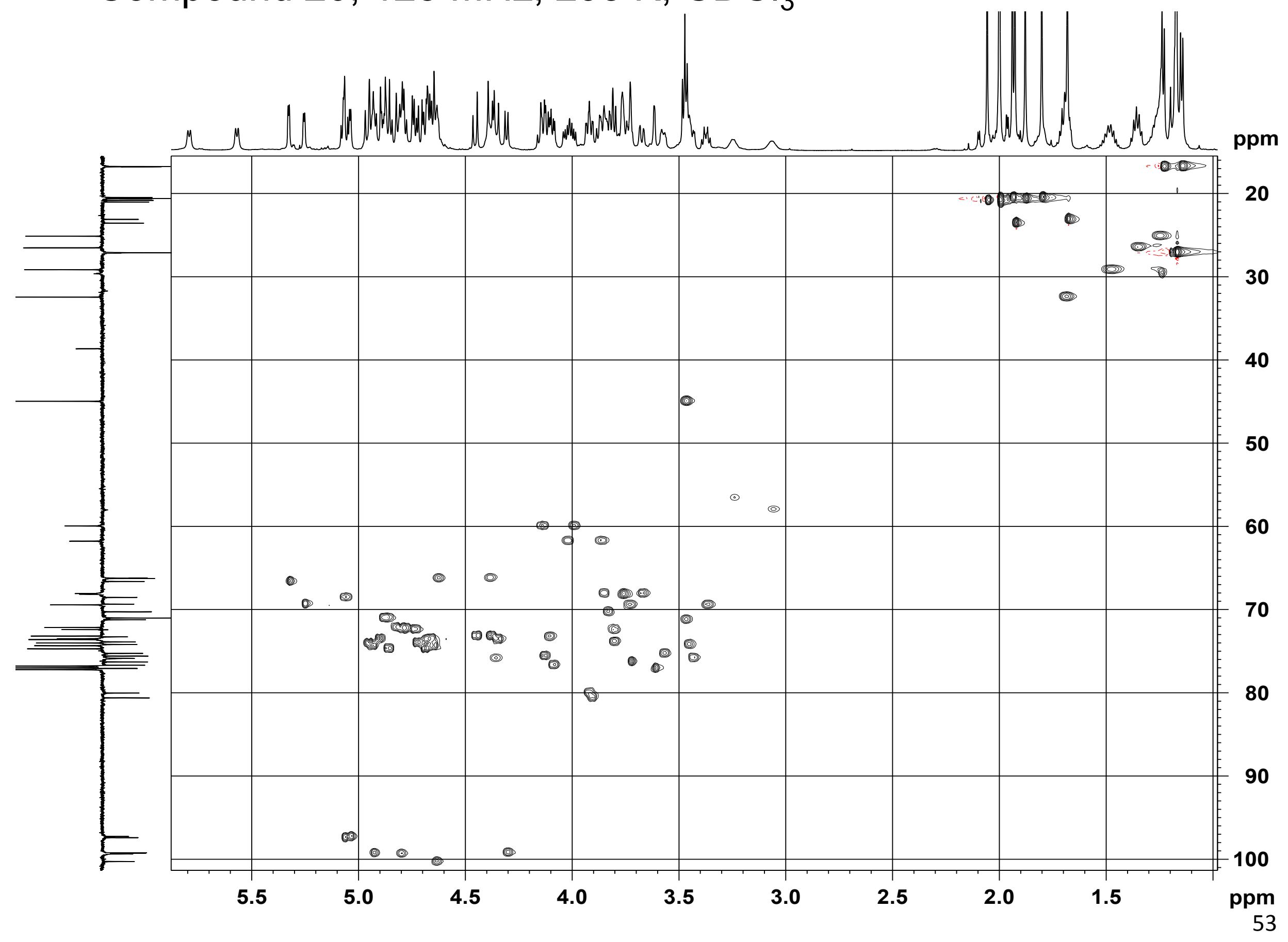




\section{Compound 21, $600 \mathrm{MHz}, 295 \mathrm{~K}, \mathrm{CDCl}_{3}$}

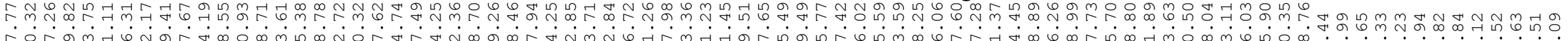

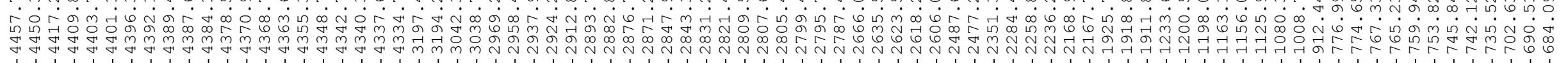

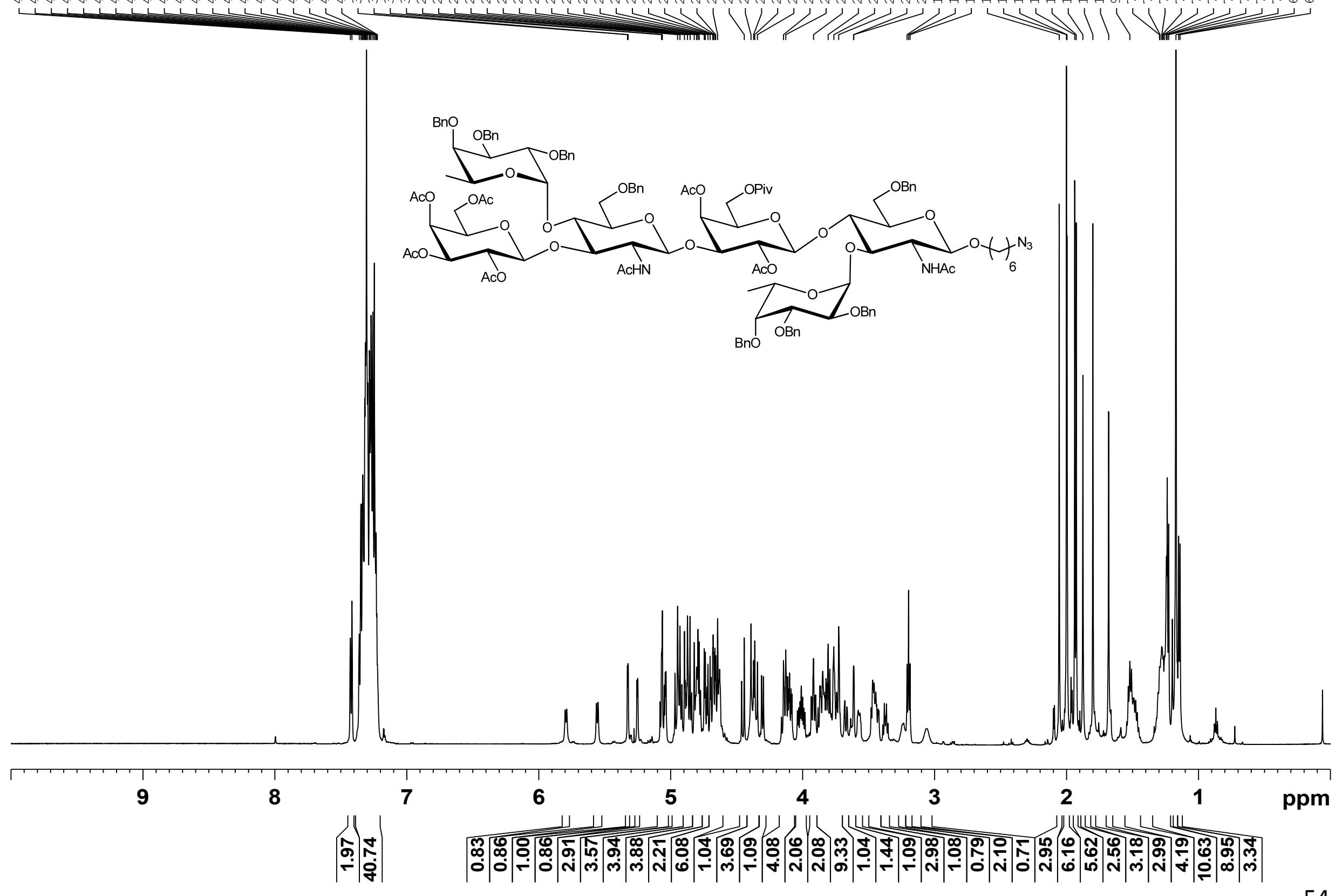


Compound 21, $600 \mathrm{MHz}, 295 \mathrm{~K}, \mathrm{CDCl}_{3}$

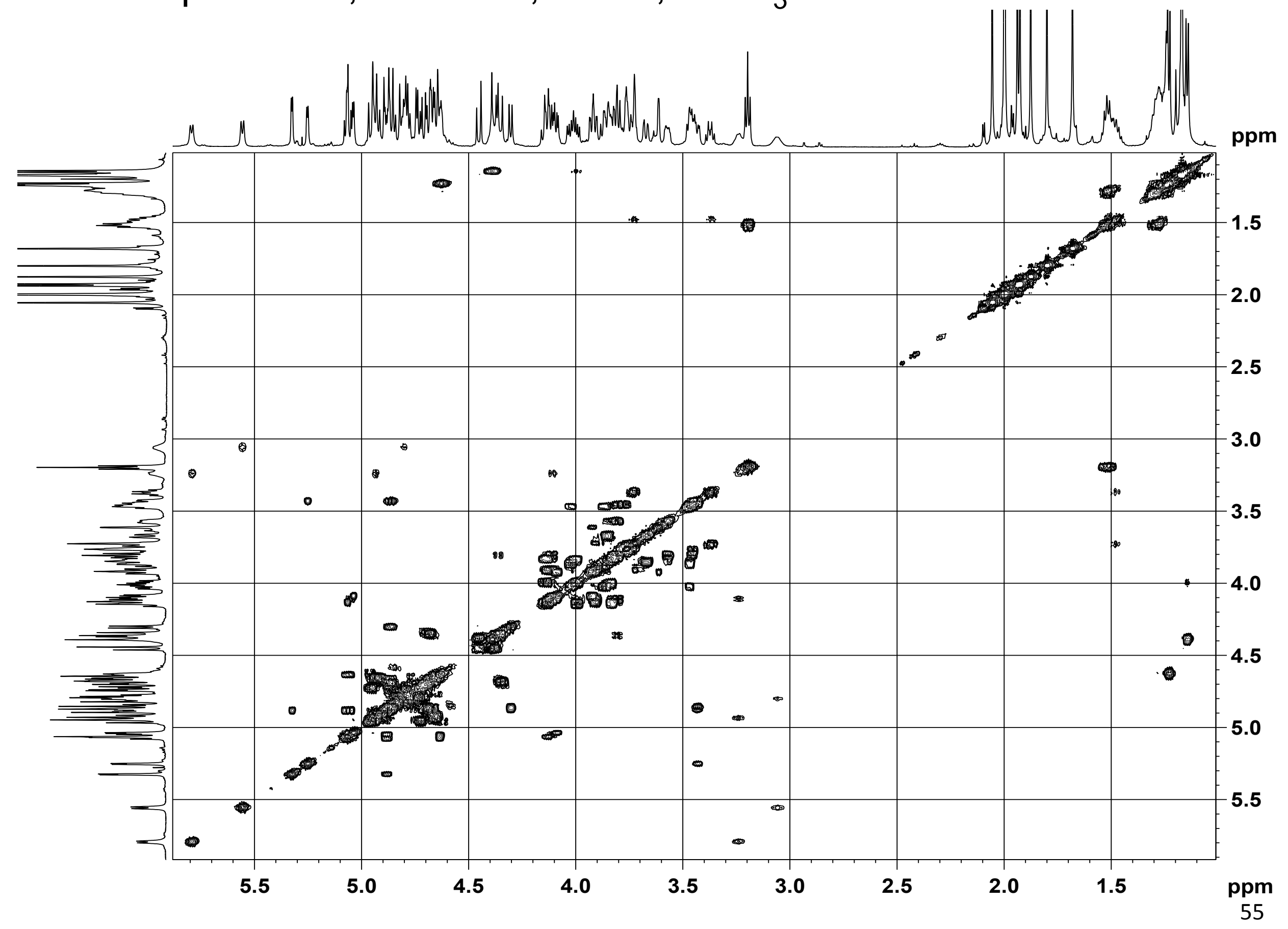




\section{Compound 21, $125 \mathrm{MHz}, 295 \mathrm{~K}, \mathrm{CDCl}_{3}$}

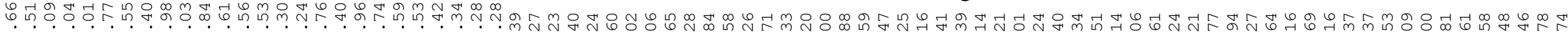

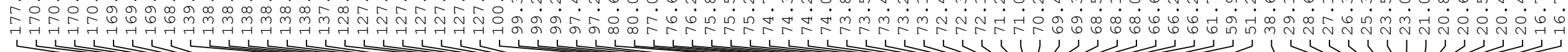

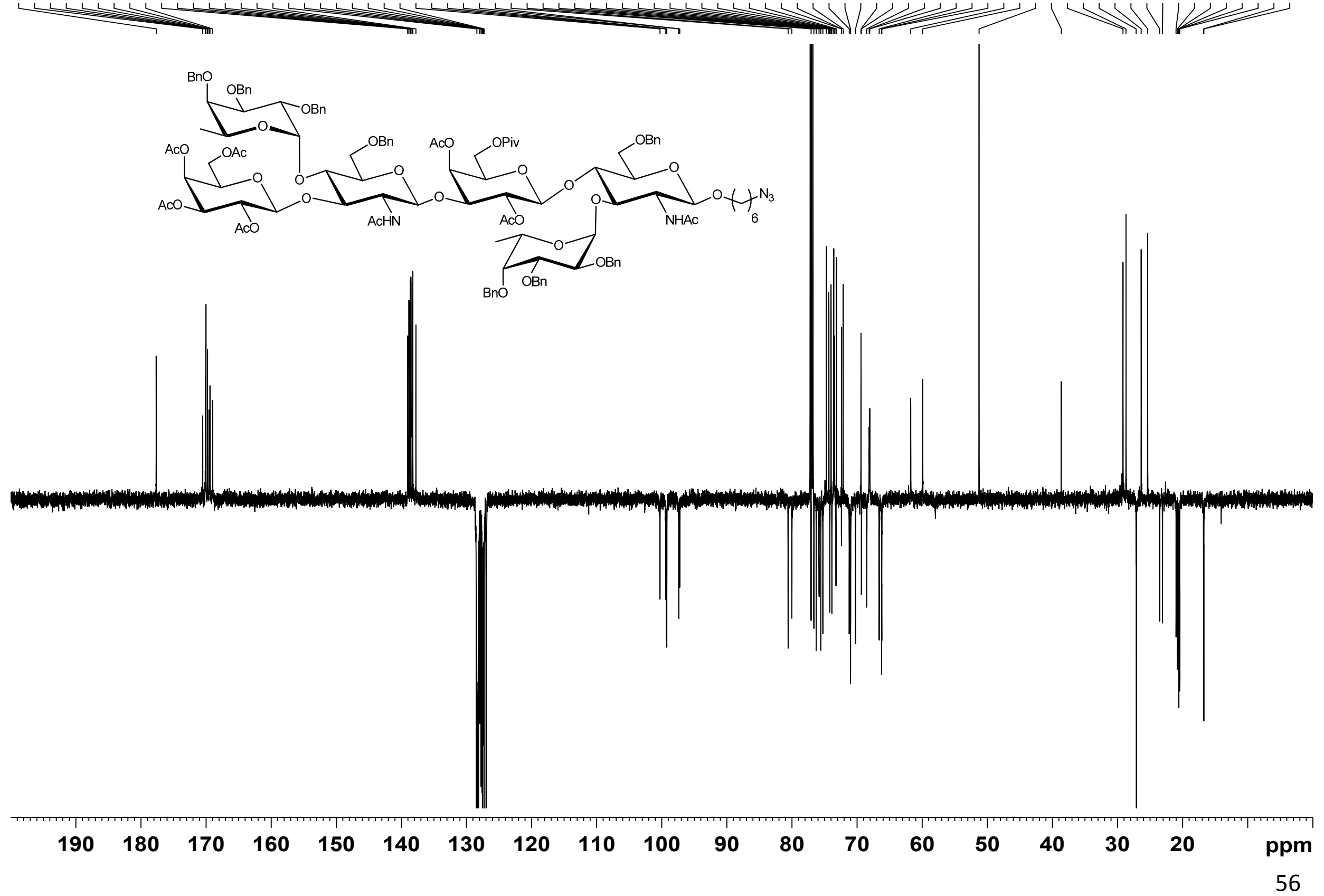


Compound 21, $125 \mathrm{MHz}, 295 \mathrm{~K}, \mathrm{CDCl}_{3}$

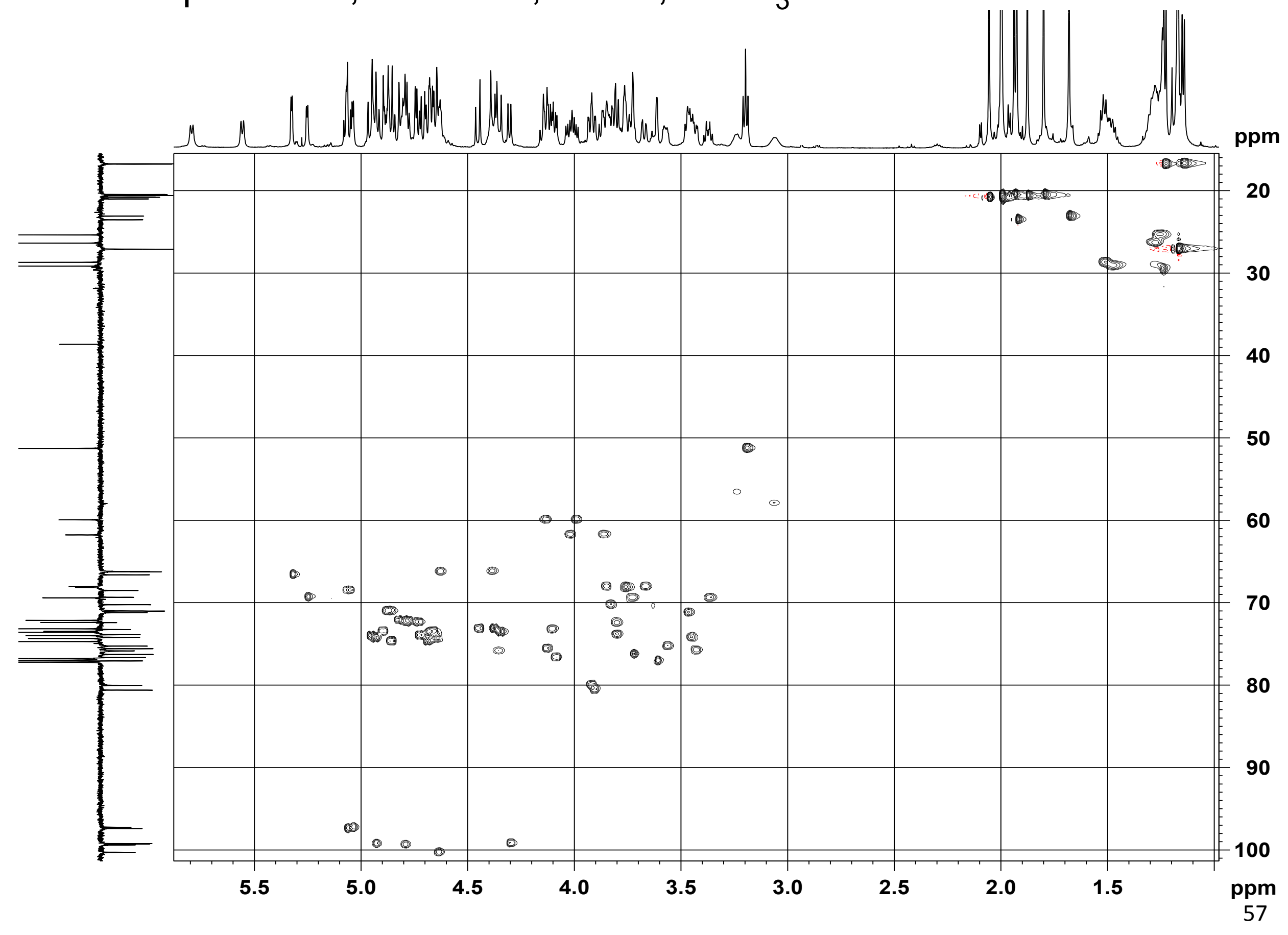




\section{Compound 22, $600 \mathrm{MHz}, 295 \mathrm{~K}, \mathrm{CDCl}_{3}$}

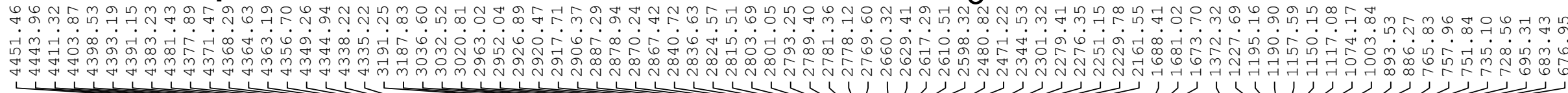

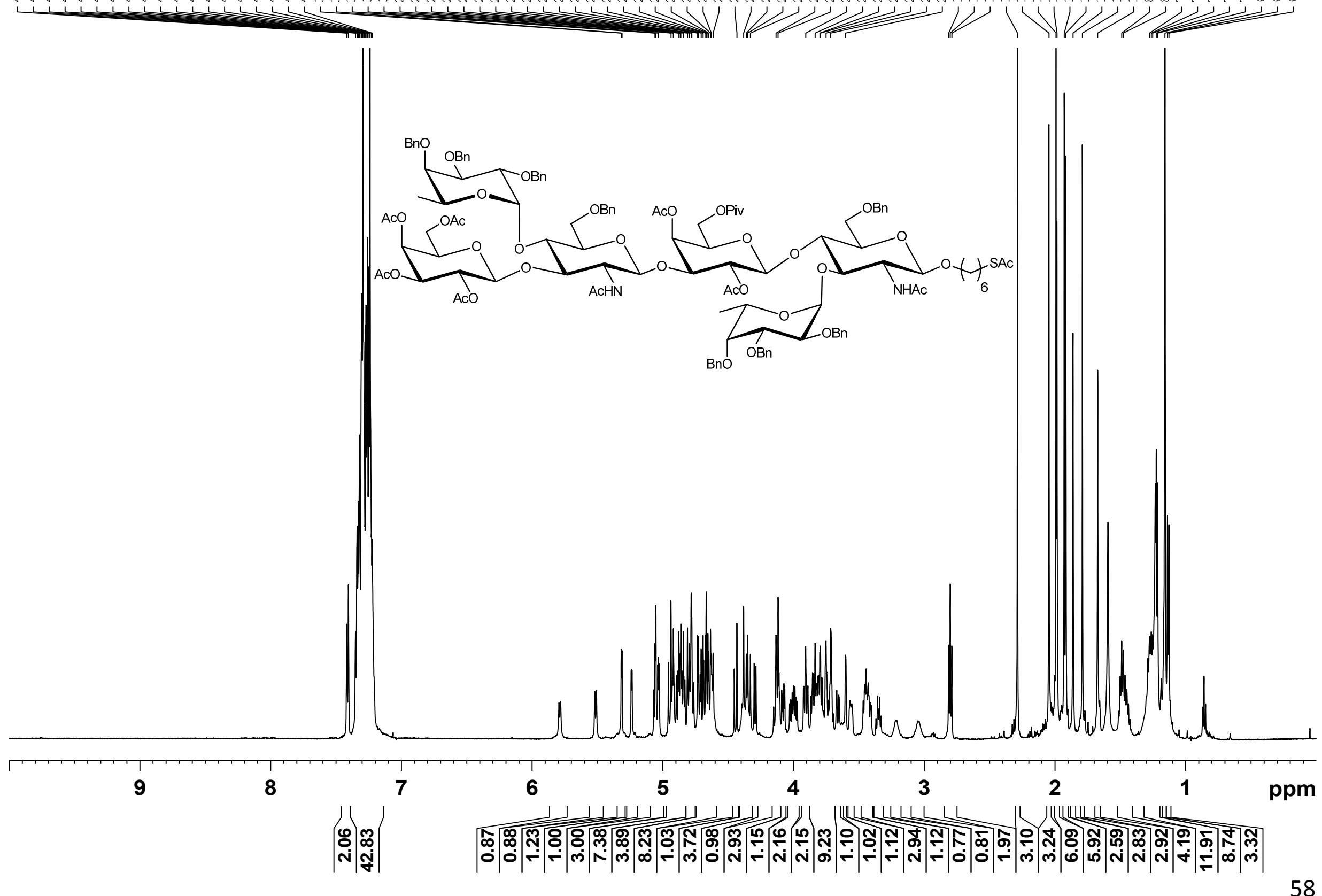


Compound 22, $600 \mathrm{MHz}, 295 \mathrm{~K}, \mathrm{CDCl}_{3}$

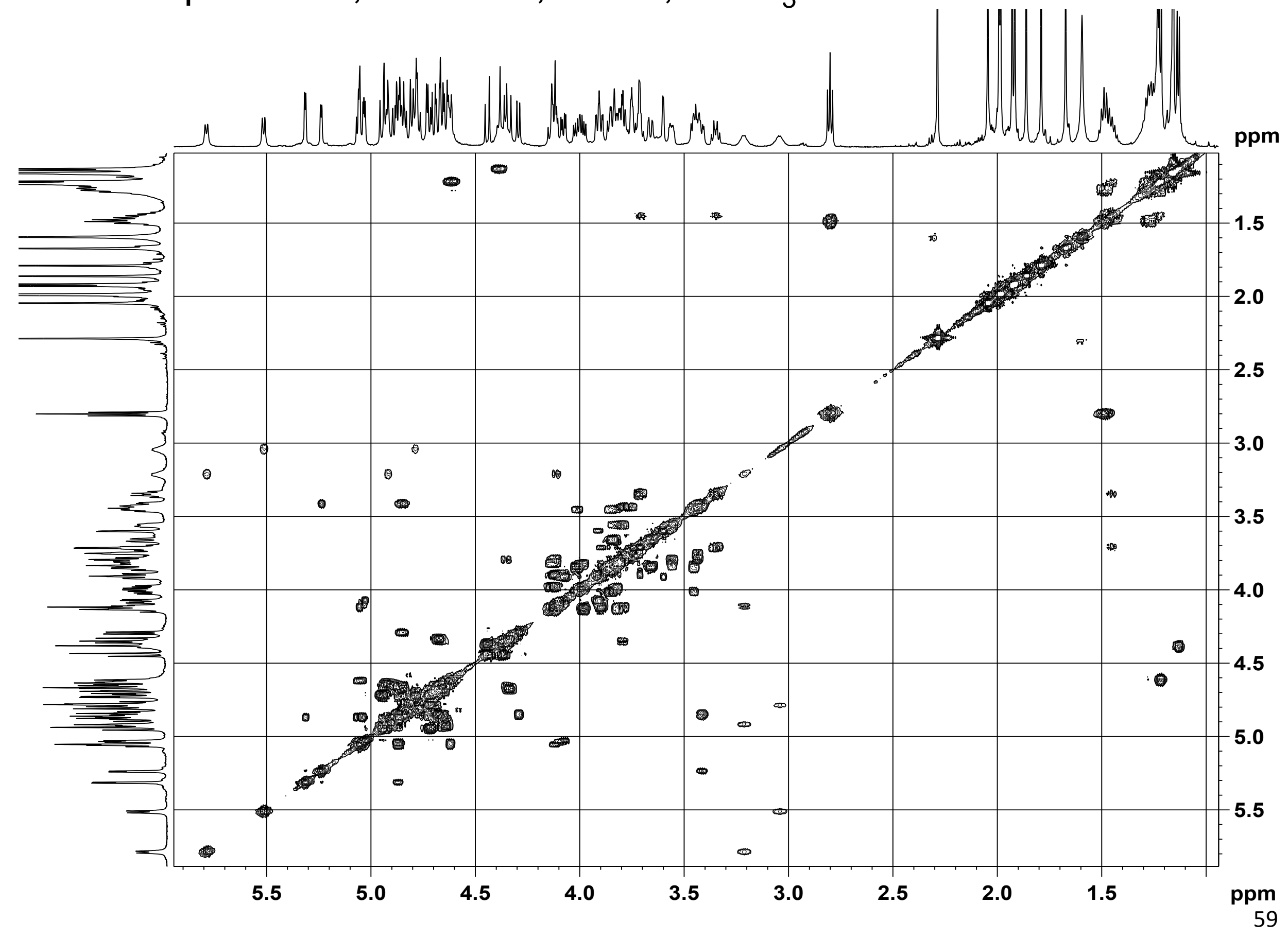




\section{Compound 22, $125 \mathrm{MHz}, 295 \mathrm{~K}, \mathrm{CDCl}_{3}$}

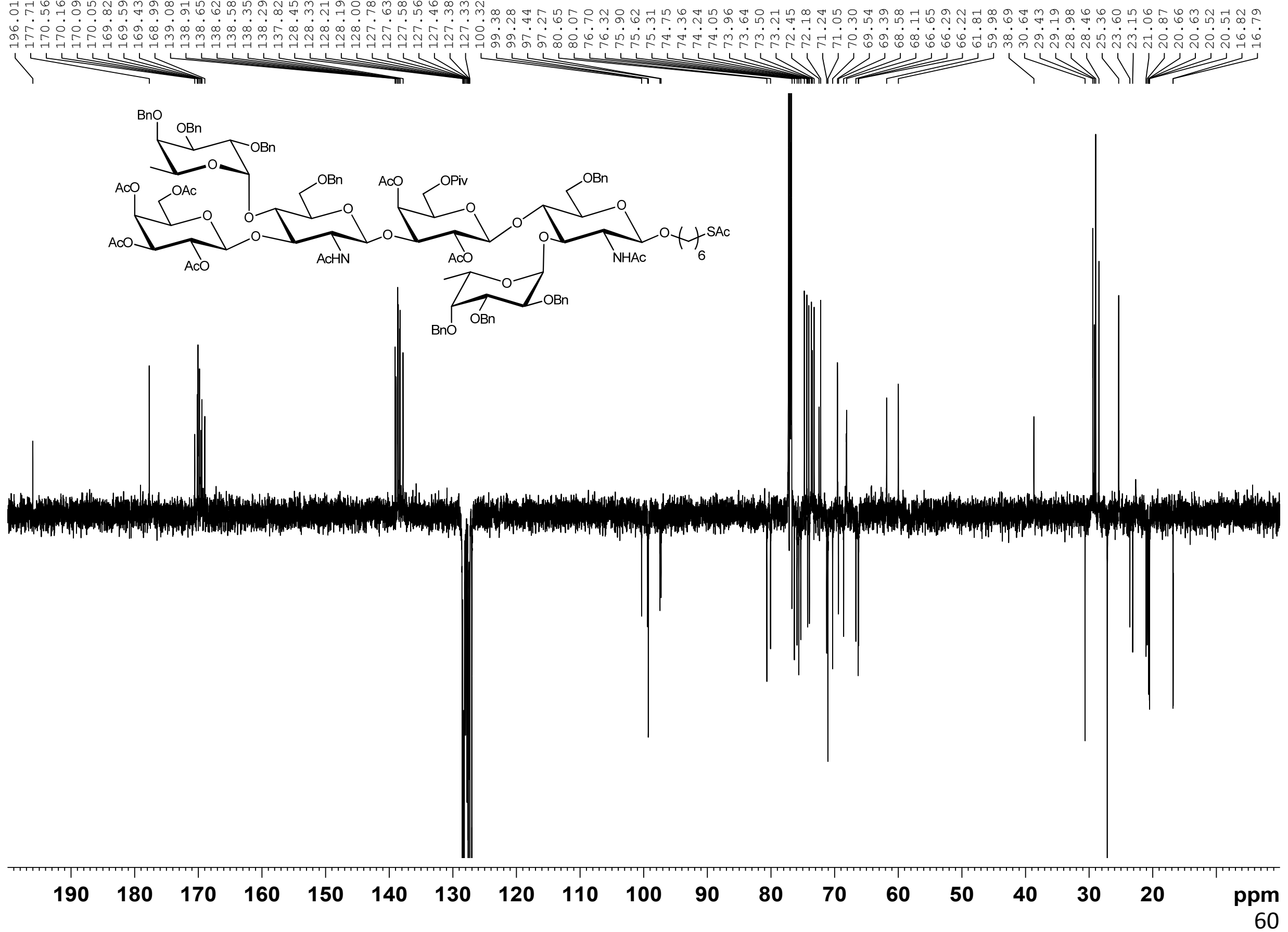


Compound 22, $125 \mathrm{MHz}, 295 \mathrm{~K}, \mathrm{CDCl}_{3}$

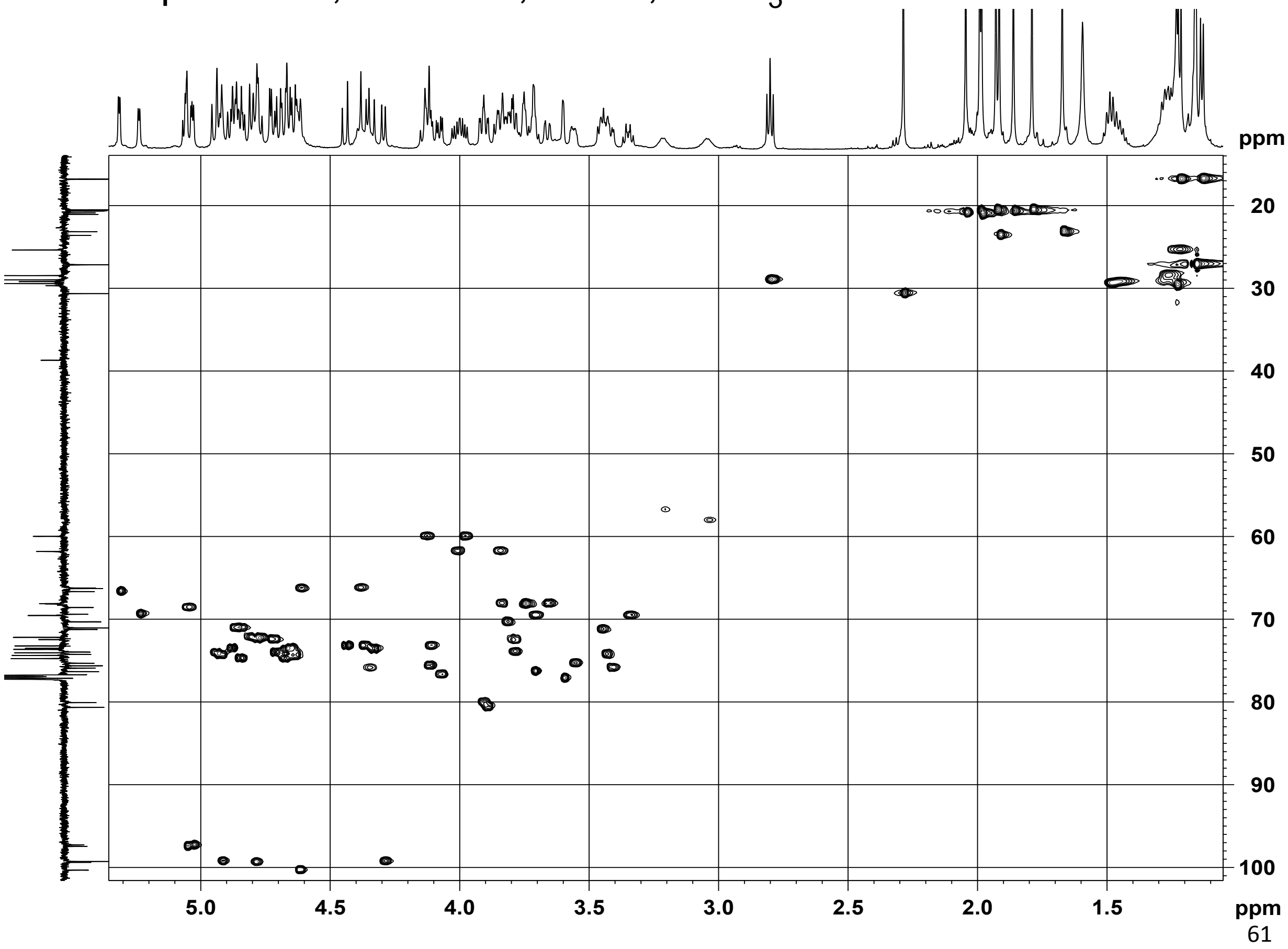




\section{Compound 2, $600 \mathrm{MHz}, 295 \mathrm{~K}, \mathrm{D}_{2} \mathrm{O}$}

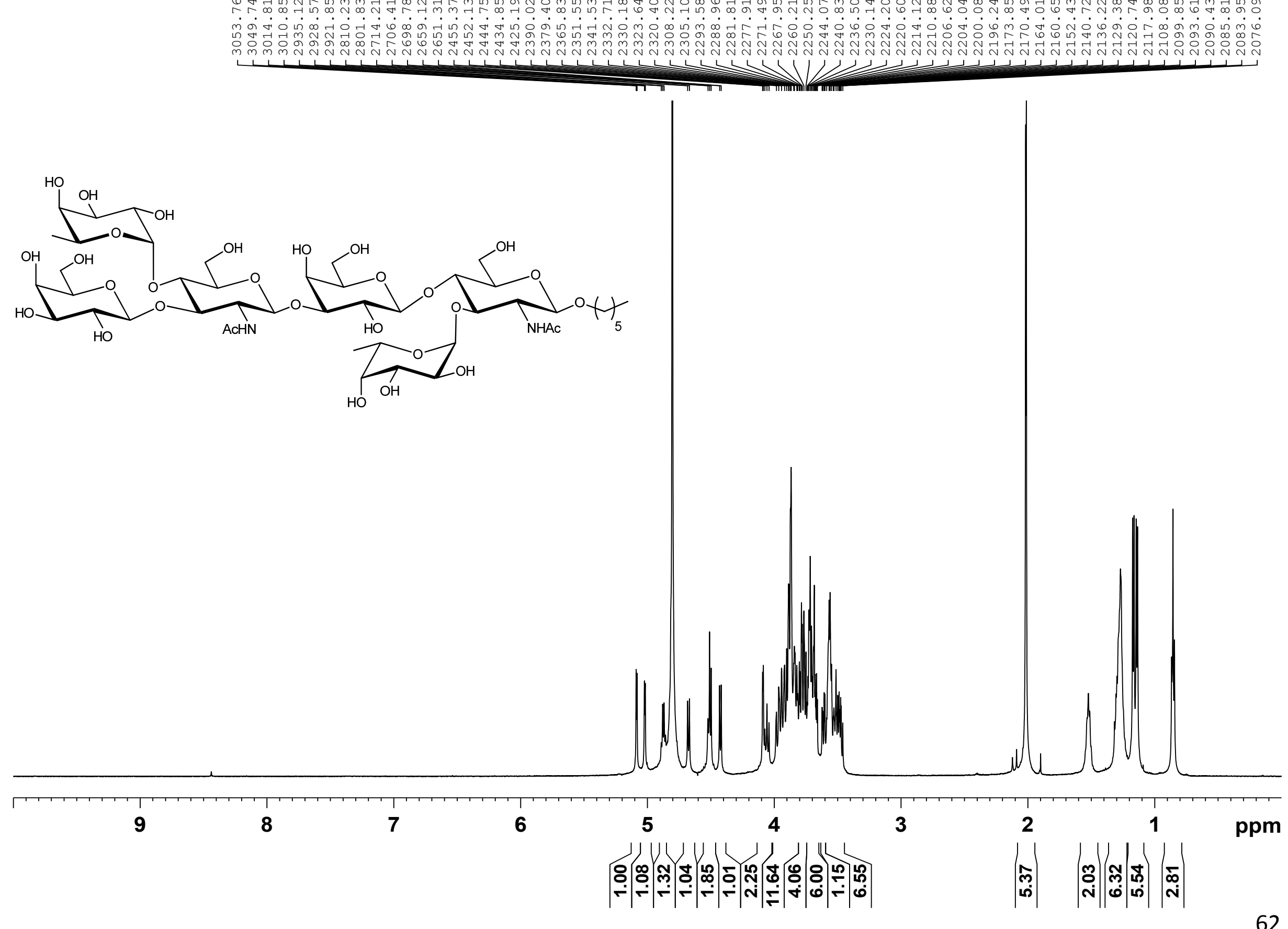


Compound 2, $600 \mathrm{MHz}, 295 \mathrm{~K}, \mathrm{D}_{2} \mathrm{O}$

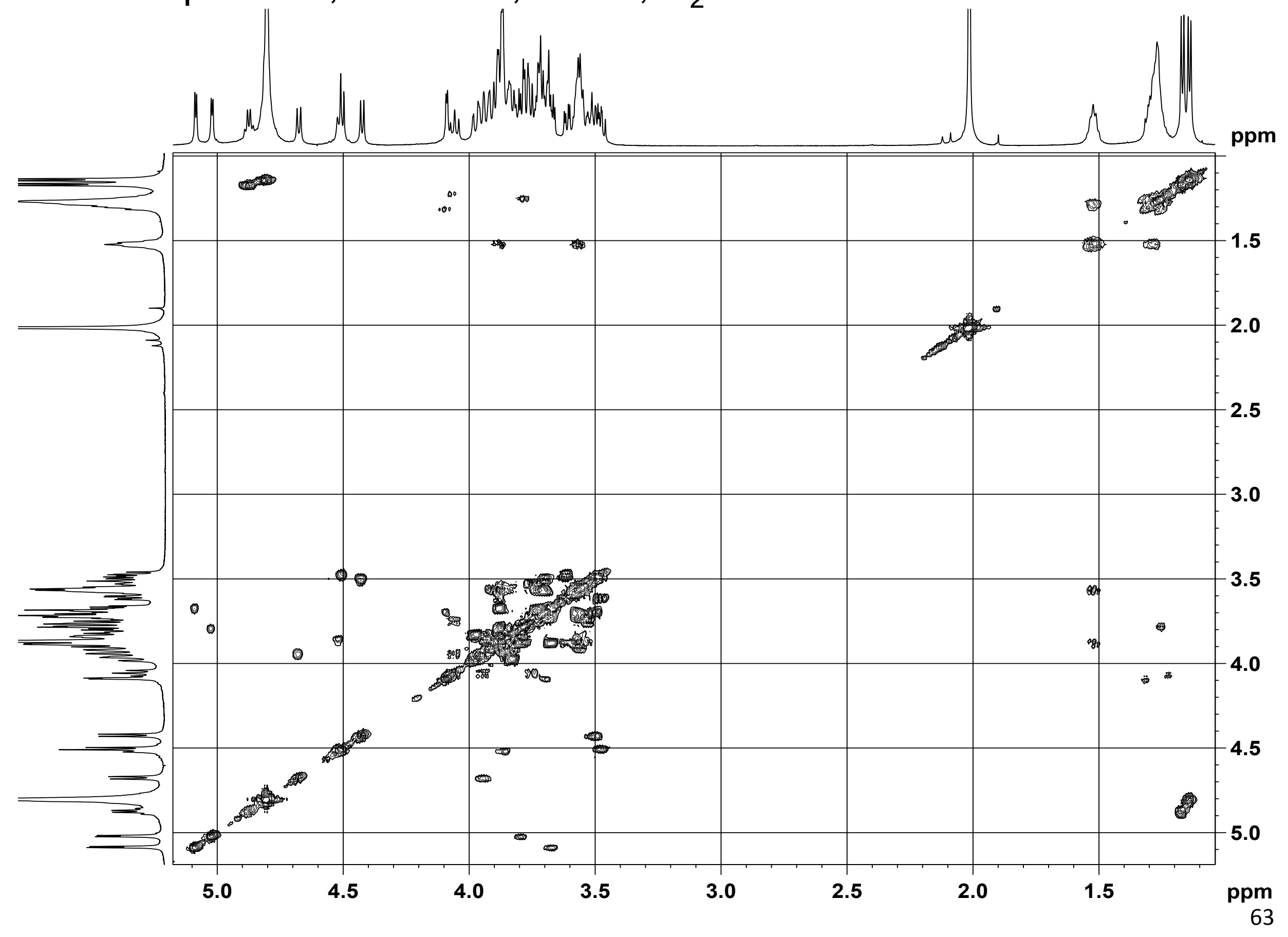


Compound 2, $125 \mathrm{MHz}, 295 \mathrm{~K}, \mathrm{D}_{2} \mathrm{O}$

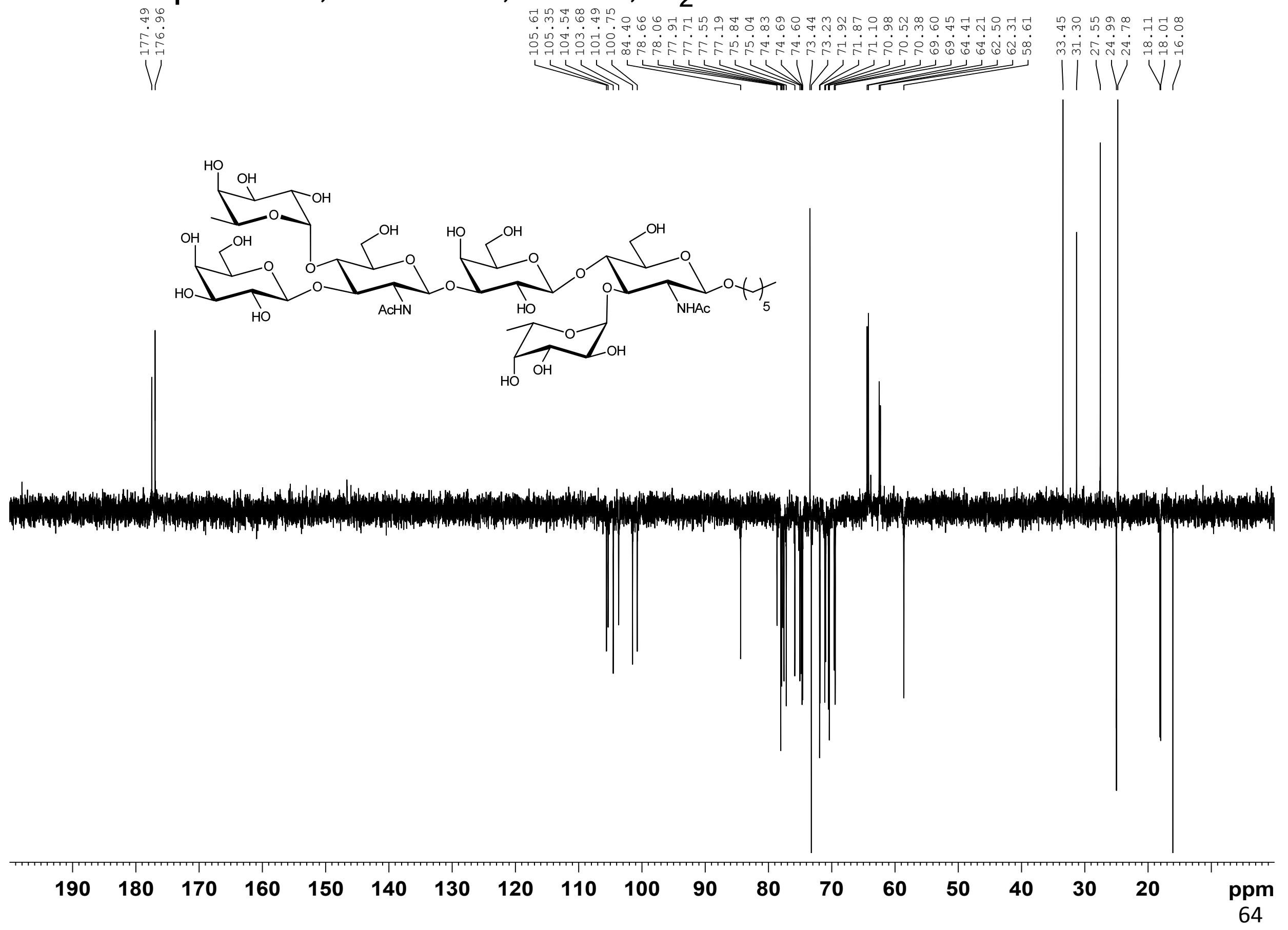


Compound 2, $125 \mathrm{MHz}, 295 \mathrm{~K}, \mathrm{D}_{2} \mathrm{O}$

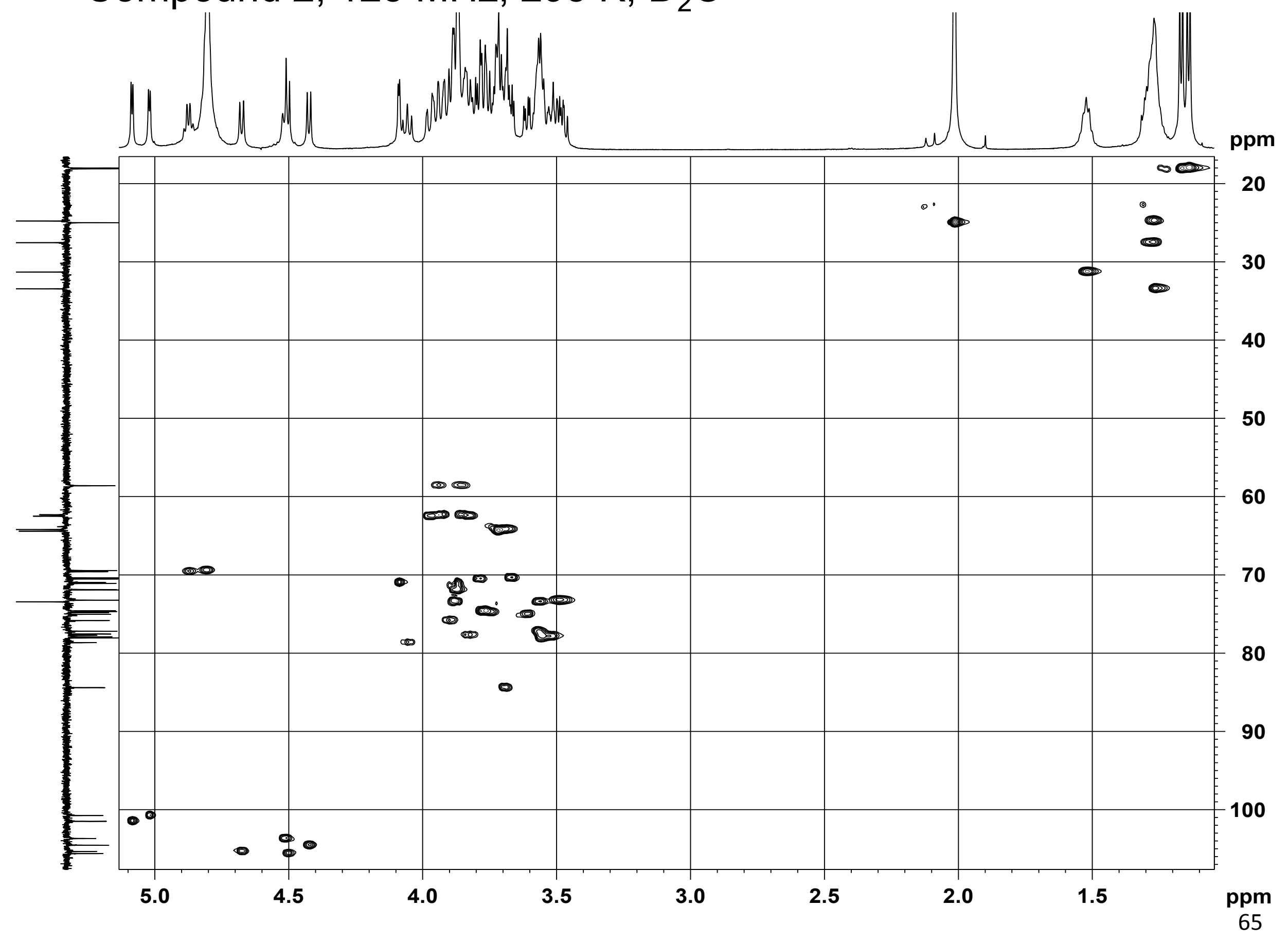




\section{Compound 3, $600 \mathrm{MHz}, 295 \mathrm{~K}, \mathrm{D}_{2} \mathrm{O}$}

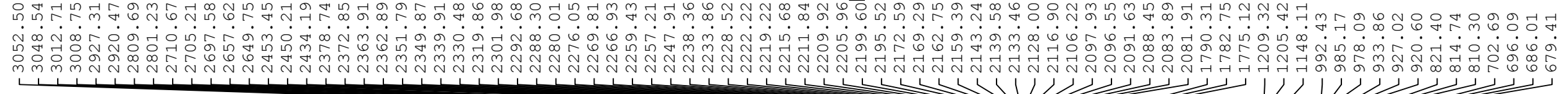

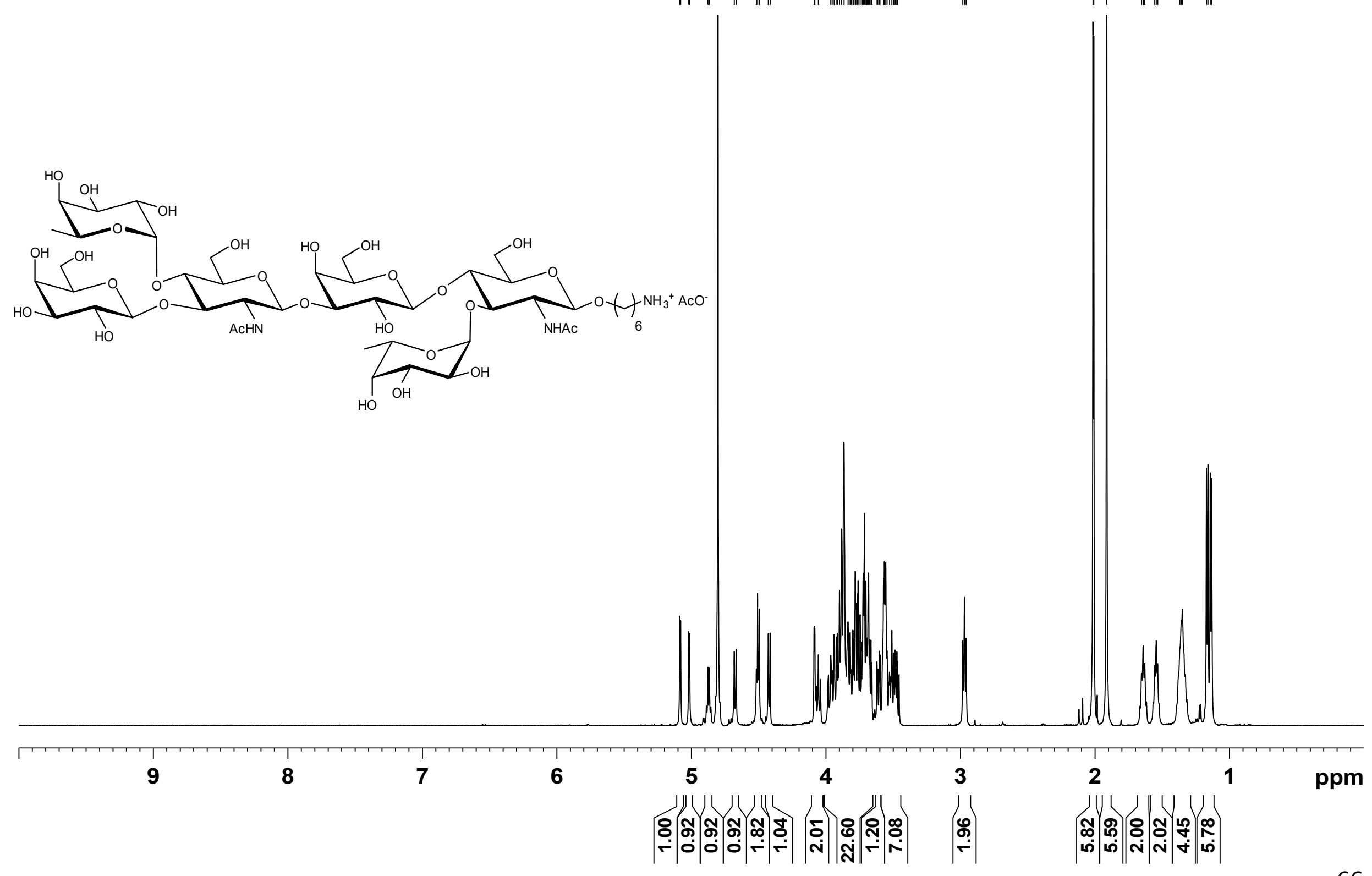


Compound 3, $600 \mathrm{MHz}, 295 \mathrm{~K}, \mathrm{D}_{2} \mathrm{O}$

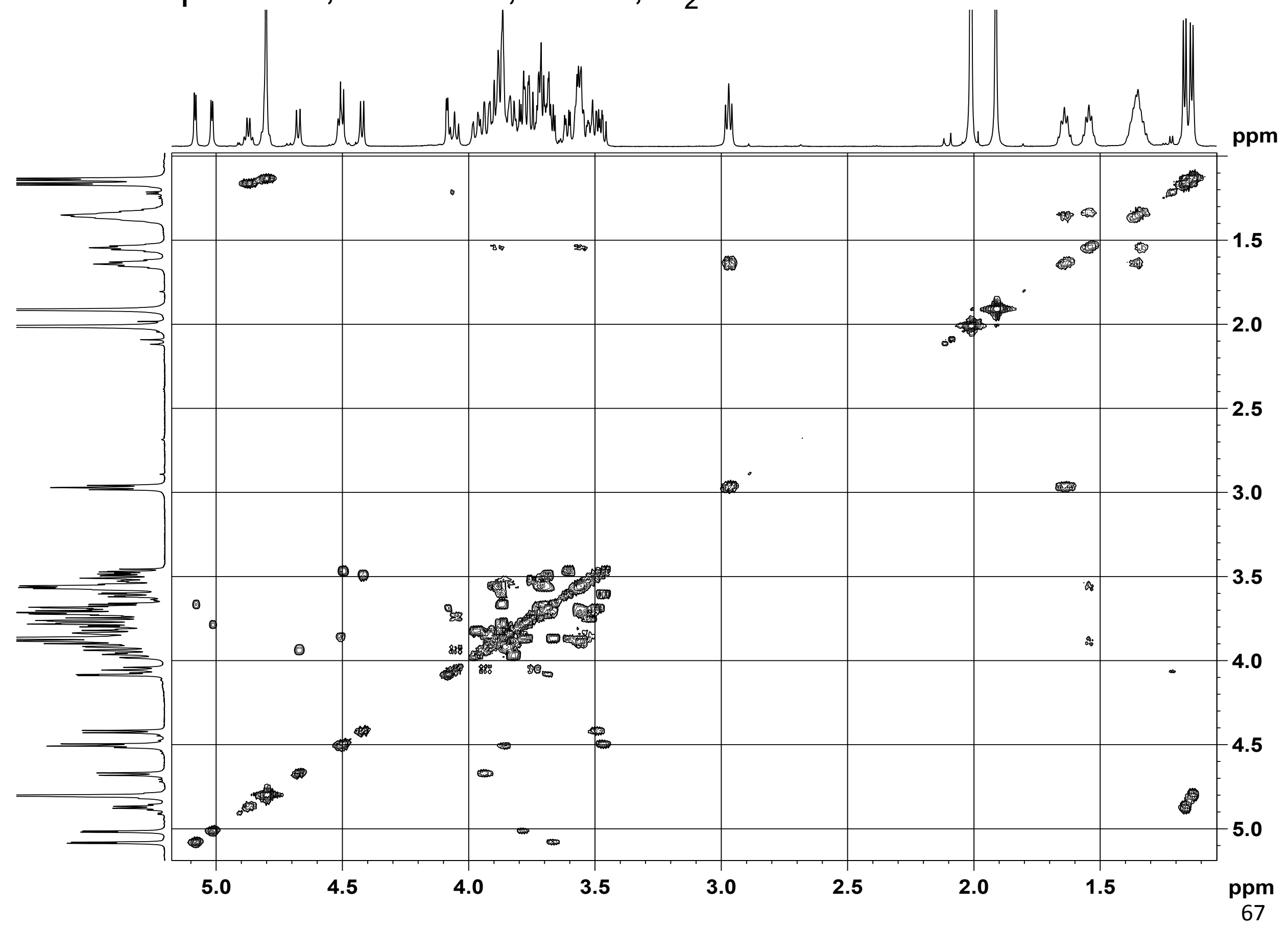


Compound 3, $125 \mathrm{MHz}, 295 \mathrm{~K}, \mathrm{D}_{2} \mathrm{O}$

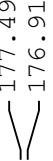
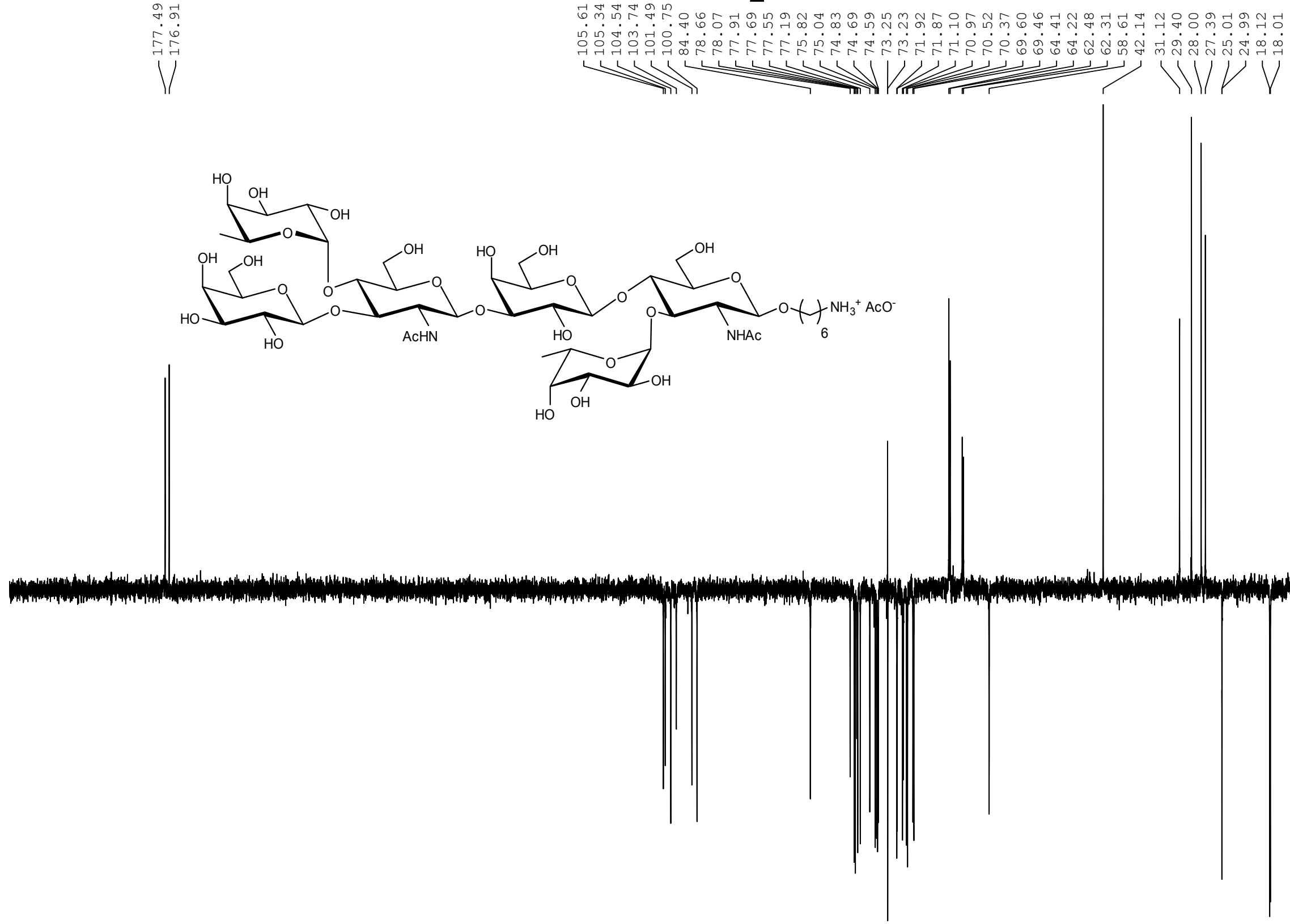
Compound 3, $125 \mathrm{MHz}, 295 \mathrm{~K}, \mathrm{D}_{2} \mathrm{O}$

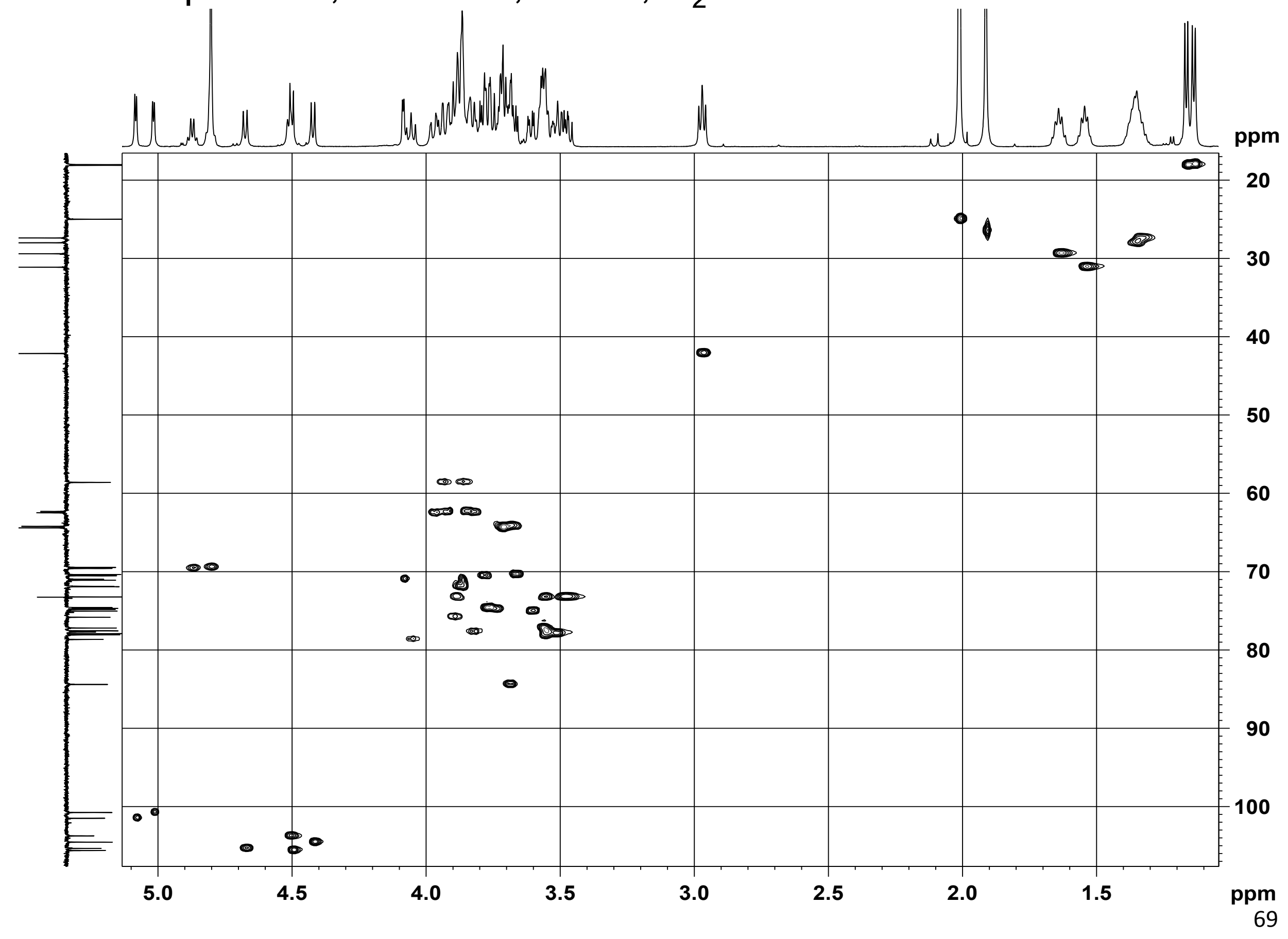




\section{Compound 4, $600 \mathrm{MHz}, 295 \mathrm{~K}, \mathrm{D}_{2} \mathrm{O}$}

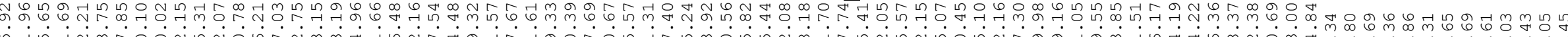

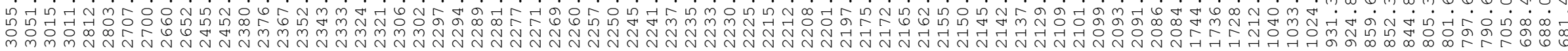

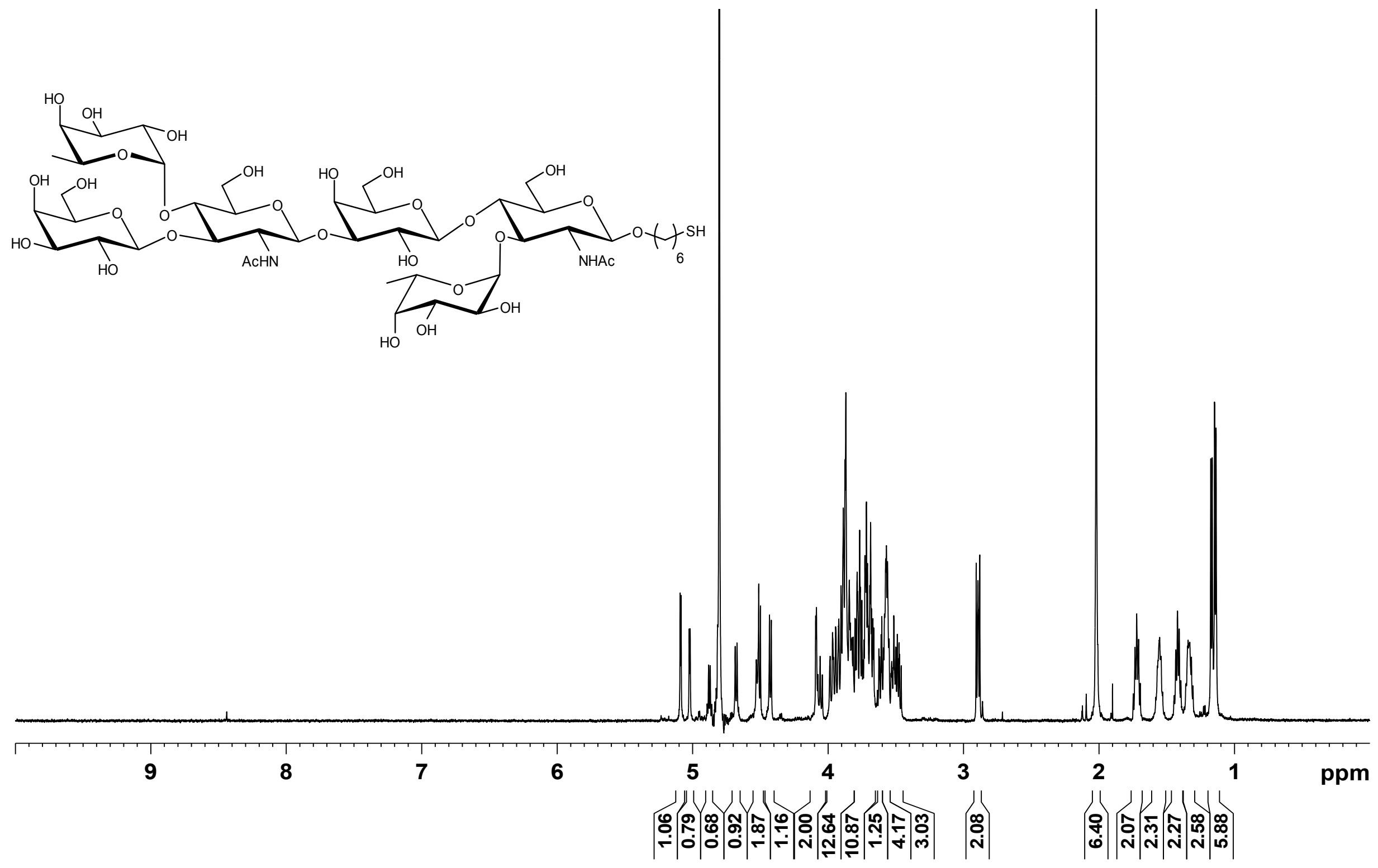


Compound 4, $600 \mathrm{MHz}, 295 \mathrm{~K}, \mathrm{D}_{2} \mathrm{O}$

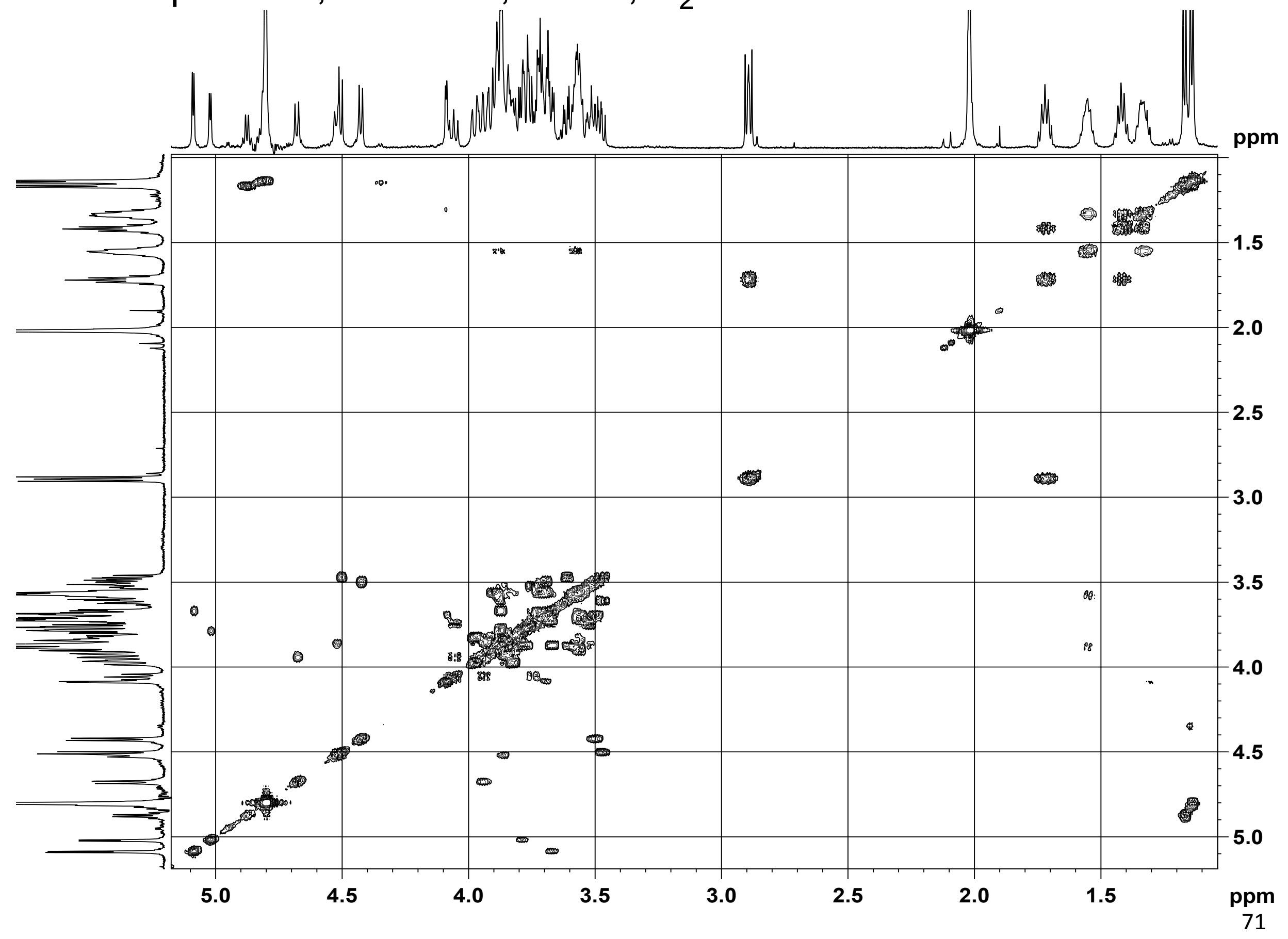


Compound 4, $125 \mathrm{MHz}, 295 \mathrm{~K}, \mathrm{D}_{2} \mathrm{O}$

V
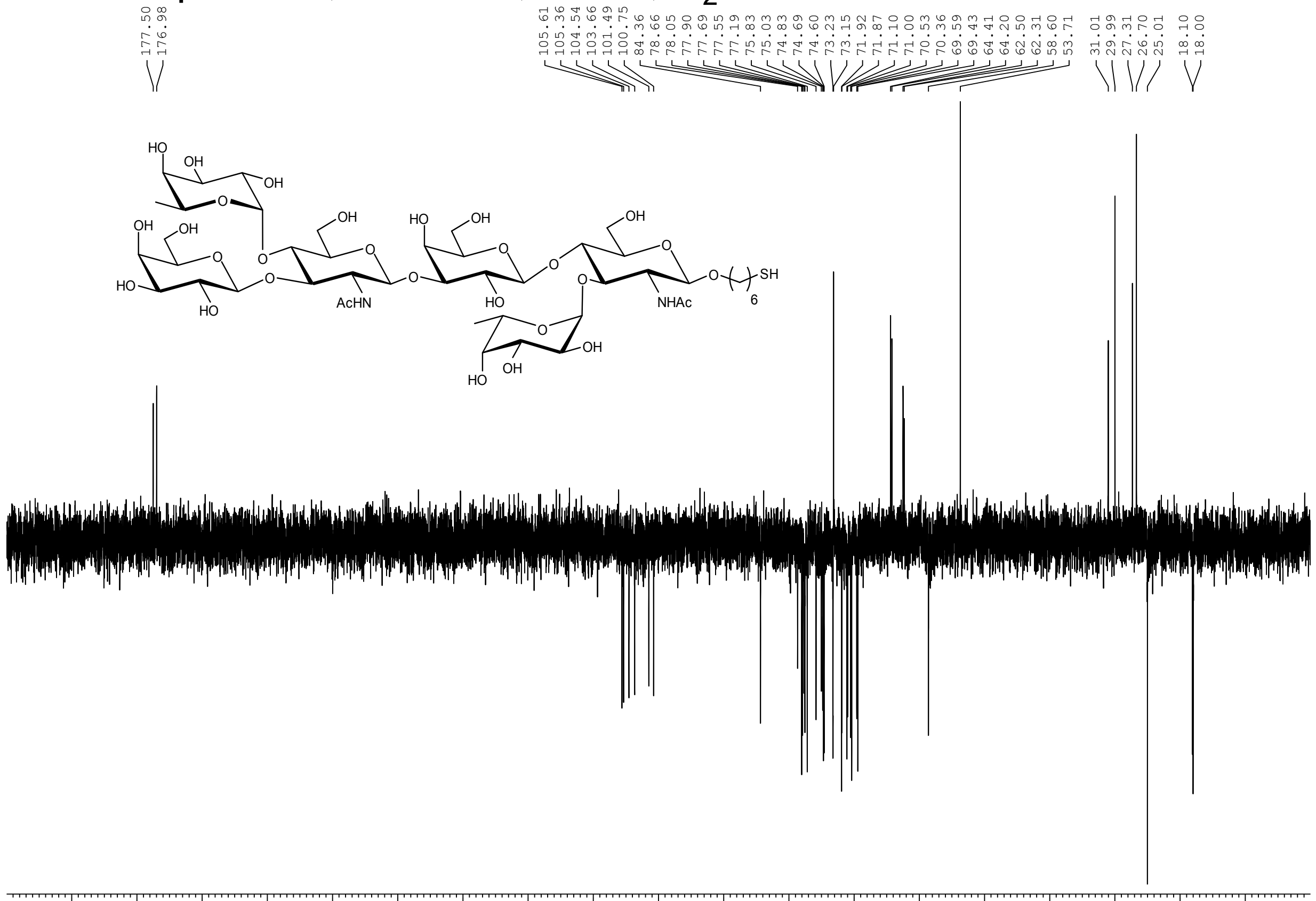
Compound 4, $125 \mathrm{MHz}, 295 \mathrm{~K}, \mathrm{D}_{2} \mathrm{O}$

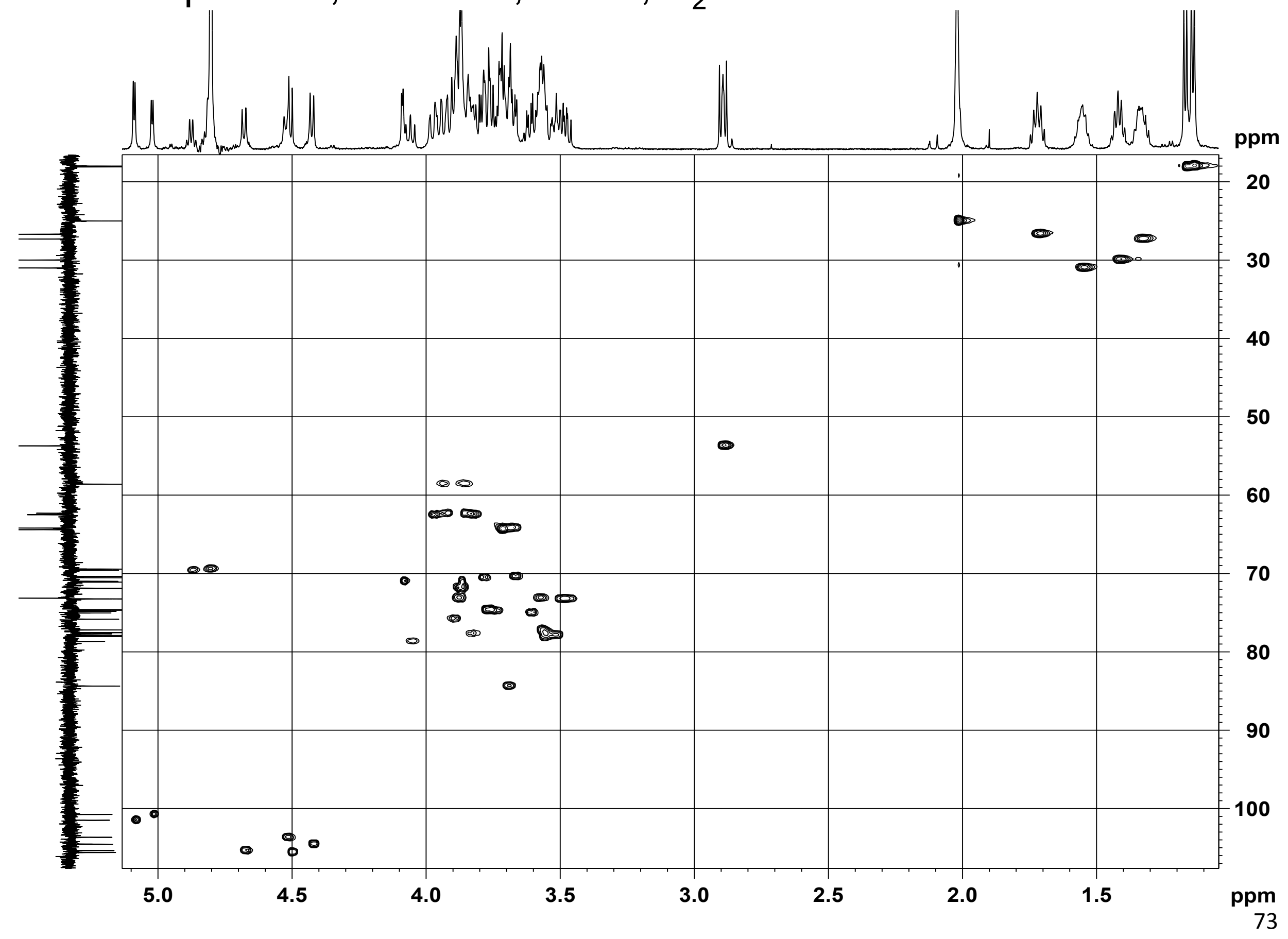

